\title{
SUPERCONVERGENT DISCONTINUOUS GALERKIN METHODS FOR NONLINEAR ELLIPTIC EQUATIONS
}

\author{
SANGITA YADAV, AMIYA K. PANI, AND EUN-JAE PARK
}

\begin{abstract}
Based on the analysis of Cockburn et al. [Math. Comp. 78 (2009), pp. 1-24] for a selfadjoint linear elliptic equation, we first discuss superconvergence results for nonselfadjoint linear elliptic problems using discontinuous Galerkin methods. Further, we have extended our analysis to derive superconvergence results for quasilinear elliptic problems. When piecewise polynomials of degree $k \geq 1$ are used to approximate both the potential as well as the flux, it is shown, in this article, that the error estimate for the discrete flux in $L^{2}$-norm is of order $k+1$. Further, based on solving a discrete linear elliptic problem at each element, a suitable postprocessing of the discrete potential is developed and then, it is proved that the resulting post-processed potential converges with order of convergence $k+2$ in $L^{2}$-norm. These results confirm superconvergent results for linear elliptic problems.
\end{abstract}

\section{INTRODUCTION}

In this article, we discuss superconvergent discontinuous Galerkin methods for the following nonlinear elliptic equations:

$$
\left\{\begin{aligned}
-\nabla \cdot(a(u) \nabla u) & =f & & \text { in } \Omega \\
u & =0 & & \text { on } \partial \Omega,
\end{aligned}\right.
$$

where $\Omega$ is a convex bounded domain in $\mathbb{R}^{2}$ with smooth boundary $\partial \Omega$. We assume that there exist positive constants $\alpha_{0}, M$ such that $0<\alpha_{0} \leq a(x, u) \leq M, a(\cdot, \cdot)$ is a twice continuously differentiable function in $\bar{\Omega} \times \mathbb{R}$ and all the derivatives of $a(\cdot, \cdot)$ through second order are bounded in $\bar{\Omega} \times \mathbb{R}$.

In literature, various DG formulations have appeared for approximating solutions of linear elliptic problems; see 3 . The authors of $[3$ have shown that approximations of the potential and the flux given by consistent and stable DG methods converge in $L^{2}$-norm with order $k+1$ and $k$, respectively, for any $k \geq 1$, when piecewise polynomials of degree $k \geq 1$ are used as approximating spaces. The performance of representative DG methods are compared in [8]. One such DG method is the local discontinuous Galerkin (LDG) method, which was originally initiated for a system of first order hyperbolic problems. Cockburn and Shu [16 have discussed the LDG method for time dependent convection-diffusion problems. The method is then extended to elliptic problems for a mixed discontinuous Galerkin formulation; see [9]. In [9], the authors have discussed stability and order of convergence of the LDG method applied to the Laplace equation. With a specific choice of numerical fluxes, it is shown in [9] that the discrete potential $u_{h}$ and its flux $\mathbf{q}_{h}$ converge in

Received by the editor October 29, 2010 and, in revised form, November 15, 2011. 2010 Mathematics Subject Classification. Primary 65-XX. 
$L^{2}$-norm with order $k+1$ and $k$, respectively, for any $k \geq 1$ if the stabilizing parameter $\mathbf{C}_{12}$ is of order one and $C_{11}$ and $1 / C_{22}$ are of order $1 / h$. The same orders of convergence are also shown to hold, when $C_{22}=0$. It is again observed in [9] that if $C_{11}$ and $C_{22}$ are of order one, then $u_{h}$ and $\mathbf{q}_{h}$ converge in $L^{2}$-norm with order $k+1$ and $k+1 / 2$, respectively, for any $k \geq 0$. Subsequently, the authors in [19] have discussed the LDG method for quasilinear elliptic boundary value problems and they have shown that $u_{h}$ and $\mathbf{q}_{h}$ converge in $L^{2}$-norm with order $k+1$ and $k$, respectively. For the LDG method applied to nonlinear elliptic problems, we also refer to [7].

One of the DG methods, called the SF-H method [12] which deals with a superconvergence property of the flux, shows that the approximation of the flux in $L^{2}$-norm converges with order $k+1$. Later on, Cockburn et al. [15] have identified DG methods for linear selfadjoint elliptic problems which provide superconvergence results for the flux. Further, using properties of Raviart-Thomas projection and employing appropriate post-processing of the discrete flux as well as the discrete potential, it is shown that the approximate flux converges in $L^{2}$-norm with order $k+1$, when piecewise polynomial spaces of degree $k \geq 1$ are employed. Moreover, with the help of suitably chosen postprocessing of the approximation of the potential, the authors of [15] have proved that the order of convergence of the resulting postprocessing is of order $k+2$.

In this article, we extend the analysis of superconvergent DG methods for linear selfadjoint elliptic problems discussed in [15] to a second order nonlinear elliptic boundary value problem (1.1) and we derive superconvergence results for the potential as well as for the flux variables. The primary tool used in proving these error estimates is based on the linear operator

$$
M \phi=-\nabla \cdot\left(a(x, u) \nabla \phi+\phi a_{u}(x, u) \nabla u\right),
$$

obtained by linearizing the elliptic operator in (1.1) about the solution $u$ of (1.1) [17. Existence for the nonlinear system resulting from DG methods is then based on a fixed point argument which requires a priori error analysis of a Dirichlet problem for the operator $M$ (see, for example, 20, 21] in the context of the mixed method). In the first part of this article, the analysis of Cockburn et al. [15] is extended to DG methods applied to a second order linear nonselfadjoint elliptic problem and using the solution of the linear selfadjoint elliptic problem as the intermediate solution, superconvergence results are proved. One of the key ingredients used, as in [15], is an introduction of a new approximation to the flux, which belongs to $H(\operatorname{div}, \Omega$ ) space and is obtained in an element-by-element fashion by employing a modification of the RT projection. In the second part, using intermediate projections as solutions of the associated linearized problem, superconvergence results for the gradient and the flux in $L^{2}$-norm are proved for the nonlinear elliptic problem (1.1). Finally, with help of a suitable postprocessing of the approximation to the potential, which results in solving a linear elliptic problem, a superconvergence result for the potential is derived. We note that intermediate projections facilitate the error analysis for nonlinear elliptic problems (see [17]-[19], 20]-21] and for parabolic problems [23]), where an elliptic projection as an intermediate projection was first introduced.

With $u_{h}, \mathbf{q}_{h}$ and $\boldsymbol{\sigma}_{h}$ defined in Section 4 as the corresponding DG solutions, we now state, below, the main result of this paper. 
Theorem 1.1. There exists a constant $C$ such that for sufficiently small $h$ and $k \geq 1$, the following estimate holds:

$$
\left\|u-u_{h}\right\|+\left\|\mathbf{q}-\mathbf{q}_{h}\right\|+\left\|\boldsymbol{\sigma}-\boldsymbol{\sigma}_{h}\right\| \leq C h^{k+1} .
$$

With a suitable postprocessing $u_{h}^{*}$ of $u_{h}$ which rely on solving a linear elliptic problem on each element, it is also shown in Theorem 5.1 that

$$
\left\|u-u_{h}^{*}\right\| \leq C h^{k+2} \text {. }
$$

Throughout this paper, we denote by $C$, a positive generic constant which does not depend on the mesh parameter $h$, but may vary from time to time.

This article is organized as follows. In Section 2, preliminaries and basic results are discussed. Section 3 is devoted to DG methods for linear nonselfadjoint elliptic problems and superconvergence results are also derived. In Section 4, DG methods are applied to quasi-linear elliptic problems and existence of a solution to the discrete nonlinear system is proved using a fixed point argument. Moreover, a priori error estimates are proved for the nonlinear elliptic problem. Further, superconvergence results for approximating the potential as well as the flux are established in section 5. Numerical experiments are performed in Section 6 to support the theoretical results. Finally, in Section 7, we summarize our results.

\section{Preliminaries}

Let $\mathcal{T}_{h}=\left\{K_{i}: 1 \leq i \leq N_{h}\right\}$ be a shape regular finite element subdivision of $\Omega$, where $K_{i}$ is either a triangle or a rectangle. Let $h_{i}$ be the diameter of $K_{i}$ and $h=\max \left\{h_{i}: 1 \leq i \leq N_{h}\right\}$. We denote the set of interior edges of $\mathcal{T}_{h}$ by $\Gamma_{I}=\left\{e_{i j}: e_{i j}=\partial K_{i} \cap \partial K_{j},\left|e_{i j}\right|>0\right\}$ and boundary edges by $\Gamma_{\partial}=\left\{e_{i \partial}: e_{i \partial}=\right.$ $\left.\partial K_{i} \cap \partial \Omega,\left|e_{i \partial}\right|>0\right\}$, where $\left|e_{k}\right|$ denotes the one-dimensional Euclidean measure. Let $\Gamma=\Gamma_{I} \cup \Gamma_{\partial}$. Note that our definition of $e_{k}$ also includes hanging nodes along each side of the finite elements. On this subdivision $\mathcal{T}_{h}$, we define the following broken Sobolev spaces:

$$
V=\left\{v \in L^{2}(\Omega): v_{\left.\right|_{K_{i}}} \in H^{1}\left(K_{i}\right), \text { for all } K_{i} \in \mathcal{T}_{h}\right\}
$$

and

$$
\mathbf{W}=\left\{\mathbf{w} \in \mathbf{L}^{2}(\Omega): \mathbf{w}_{\left.\right|_{K_{i}}} \in \mathbf{H}^{1}\left(K_{i}\right), \text { for all } K_{i} \in \mathcal{T}_{h}\right\},
$$

where $H^{1}\left(K_{i}\right)$ is the standard Sobolev space defined on $K_{i}, \mathbf{L}^{2}(\Omega)=\left(L^{2}(\Omega)\right)^{2}$ and $\mathbf{H}^{1}\left(K_{i}\right)=\left(H^{1}\left(K_{i}\right)\right)^{2}$. We also define broken Sobolev space of order $m$ as $H^{m}\left(\Omega, \mathcal{T}_{h}\right)$ and its associated broken norm and seminorm are defined, respectively, as

$$
\|v\|_{H^{m}\left(\Omega, \mathcal{T}_{h}\right)}=\left(\sum_{i=1}^{N_{h}}\|v\|_{H^{m}\left(K_{i}\right)}^{2}\right)^{\frac{1}{2}} \text { and }|v|_{H^{m}\left(\Omega, T_{h}\right)}=\left(\sum_{i=1}^{N_{h}}|v|_{H^{m}\left(K_{i}\right)}^{2}\right)^{\frac{1}{2}} .
$$

For any $v, w \in V$, we define

$$
(v, w):=\int_{\Omega} v w d x, \quad(v, w)_{K}=\int_{K} v w d x,
$$

and for any $\mathbf{w}, \boldsymbol{\tau} \in \mathbf{W}$, we define

$$
(\mathbf{w}, \boldsymbol{\tau}):=\int_{\Omega} \mathbf{w} \cdot \boldsymbol{\tau} d x, \quad(\mathbf{w}, \boldsymbol{\tau})_{K}=\int_{K} \mathbf{w} \cdot \boldsymbol{\tau} d x
$$


We also define for any $v \in V$ and $\mathbf{w} \in \mathbf{W}$,

$$
\langle v, \mathbf{w} \cdot \boldsymbol{\nu}\rangle=\sum_{i=1}^{N_{h}} \int_{\partial K_{i}} v \mathbf{w} \cdot \boldsymbol{\nu} d s, \quad\langle v, \mathbf{w} \cdot \boldsymbol{\nu}\rangle_{\partial K}=\int_{\partial K_{i}} v \mathbf{w} \cdot \boldsymbol{\nu} d s .
$$

We denote the $L^{2}$-norm by $\|$.$\| .$

Let $e_{k} \in \Gamma_{I}$, that is, $e_{k}=\partial K_{i} \cap \partial K_{j}$ for some $i$ and $j$. Let $\boldsymbol{\nu}_{i}$ and $\boldsymbol{\nu}_{j}$ be the outward normals to the boundary $\partial K_{i}$ and $\partial K_{j}$, respectively. On $e_{k}$, we now define the jump and average of $v \in V$ as

$$
\llbracket v \rrbracket=v_{\left.\right|_{K_{i}}} \boldsymbol{\nu}_{i}+v_{\left.\right|_{K_{j}}} \boldsymbol{\nu}_{j},\{\{v\}\}=\frac{v_{\left.\right|_{K_{i}}}+v_{\left.\right|_{K_{j}}}}{2},
$$

and the jump and average of $\mathbf{w} \in \mathbf{W}$ as

$$
\llbracket \mathbf{w} \rrbracket=\mathbf{w}_{\left.\right|_{K_{i}}} \cdot \boldsymbol{\nu}_{i}+\mathbf{w}_{\left.\right|_{K_{j}}} \cdot \boldsymbol{\nu}_{j},\left\{\{\mathbf{w}\}=\frac{\mathbf{w}_{\left.\right|_{K_{i}}}+\mathbf{w}_{\left.\right|_{K_{j}}}}{2} .\right.
$$

In the case $e_{k} \in \Gamma_{\partial}$, that is, there exists $K_{i}$ such that $e_{k}=\partial K_{i} \cap \partial \Omega$, then set for notational convenience, the jump and average of $v \in V$ as

$$
\llbracket v \rrbracket=v_{\left.\right|_{i} \cap \partial \Omega} \nu,\left\{\{v\}=v_{K_{i} \cap \partial \Omega},\right.
$$

and the jump and average of $\mathbf{w} \in \mathbf{W}$ as

$$
\llbracket \mathbf{w} \rrbracket=\mathbf{w}_{\left.\right|_{K_{i} \cap \partial \Omega}} \cdot \boldsymbol{\nu},\{\{\mathbf{w}\}\}=\mathbf{w}_{\left.\right|_{K_{i} \cap \partial \Omega}},
$$

where $\boldsymbol{\nu}$ is the outward normal to the boundary $\partial \Omega$. For $\mathbf{w} \in \mathbf{W}$, we denote $\mathbf{w}^{2}=\mathbf{w} \cdot \mathbf{w}$. Let $\mathcal{P}^{p_{i}}\left(K_{i}\right)$ be the space of polynomials of total degree less than or equal to $p_{i}$ on each triangle $K_{i} \in \mathcal{T}_{h}$. The discontinuous finite element spaces are considered as

$$
V_{h}=\left\{v_{h} \in L^{2}(\Omega): v_{h \mid K_{i}} \in \mathcal{P}^{k}\left(K_{i}\right)\right\}
$$

and

$$
\mathbf{W}_{h}=\left\{\mathbf{w}_{h} \in \mathbf{L}^{2}(\Omega): \mathbf{w}_{\left.h\right|_{K_{i}}} \in \mathcal{P}^{k}\left(K_{i}\right)\right\}
$$

where $k \geq 1$.

\section{Assumption (P):}

(1) The triangulations that we consider can have hanging nodes, but have to be shape regular, that is, there exists a positive constant $\varrho_{1}$ independent of $h$ such that, for any $K_{i} \in \mathcal{T}_{h}$ and for any $e_{k} \in \partial K_{i}$, we have

$$
\varrho_{1} h_{i} \leq \ell_{k} \leq h_{i}
$$

where $\ell_{k}$ denotes the length of $e_{k}$.

(2) The finite element subdivision $\mathcal{T}_{h}$ satisfies the bounded local variation condition in the sense that if $\left|\partial K_{i} \cap \partial K_{j}\right|>0$, for any $K_{i}$ and $K_{j} \in \mathcal{T}_{h}$, then there exists a constant $\varrho_{2}$ independent of $h_{i}$ and $h_{j}$ such that

$$
\varrho_{2}^{-1} \leq \frac{h_{i}}{h_{j}} \leq \varrho_{2}
$$

In particular, it implies that for any element $K_{i}$ the number of neighboring elements $K_{j} \in T_{h}$ such that $\left|\partial K_{i} \cap \partial K_{j}\right|>0$ is bounded by $N_{\kappa}$ uniformly.

Approximation properties of the finite element spaces. Below, we state without proof a lemma on some approximation properties. 
Lemma 2.1. For $\phi \in\left(H^{s_{i}}\left(K_{i}\right)\right)^{d}, d=1,2$ there exists a positive constant $C_{A}$ (depending on $s$ but independent of $\phi, k$ and $h_{i}$ ) and a sequence $\phi_{K_{i}}^{h} \in\left(\mathcal{P}^{k}\left(K_{i}\right)\right)^{d}$, such that:

(i) for any $0 \leq l \leq s_{i}$,

$$
\left\|\phi-\phi_{K_{i}}^{h}\right\|_{H^{l}\left(K_{i}\right)^{d}} \leq C_{A} h_{i}^{\mu_{i}-l}\|\phi\|_{\left(H^{s_{i}}\left(K_{i}\right)\right)^{d}},
$$

where $\mu_{i}=\min \left\{s_{i}, k+1\right\}$.

(ii) for $s_{i}>l+\frac{1}{2}$,

$$
\left\|\phi-\phi_{K_{i}}^{h}\right\|_{H^{l}\left(e_{k}\right)^{d}} \leq C_{A} h_{i}^{\mu_{i}-l-1 / 2}\|\phi\|_{\left(H^{s_{i}}\left(K_{i}\right)\right)^{d}},
$$

where $e_{k} \in \partial K_{i}$.

(iii) for $0 \leq l \leq s_{i}-1+2 / r$,

$$
\left\|\phi-\phi_{K_{i}}^{h}\right\|_{W_{r}^{l}\left(K_{i}\right)^{d}} \leq C_{A} h_{i}^{\mu_{i}-l-1+2 / r}\|\phi\|_{\left(H^{s_{i}}\left(K_{i}\right)\right)^{d}} .
$$

The proof of properties (i) and (ii) can be found in [4. Then using properties (1) and (3) in Lemma 1 of [1] and a scaling argument (see [2]), it is easy to derive the property (iii). We now denote $I_{h}$ by

$$
I_{h} \phi_{\left.\right|_{K_{i}}}=\phi_{K_{i}}^{h}, \forall K_{i} \in \mathcal{T}_{h} .
$$

Below, we discuss some inequalities for our future use.

Lemma 2.2 (Trace Inequality, [22, Lemma 2.1]). Let $\boldsymbol{v}_{h} \in\left(\mathcal{P}^{k}\left(K_{i}\right)\right)^{d}, d=1,2$ and $e_{k} \in \partial K_{i}$. Then there exists a constant $C_{\mathcal{T}}>0$ such that

$$
\left\|\nabla^{l} \boldsymbol{v}_{h}\right\|_{L^{2}\left(e_{k}\right)^{d}} \leq C_{\mathcal{T}} h_{i}^{-\frac{1}{2}}\left\|\nabla^{l} \boldsymbol{v}_{h}\right\|_{L^{2}\left(K_{i}\right)^{d}}, l=0,1 .
$$

Lemma 2.3 (Inverse Inequality, [1]). Let $\boldsymbol{v}_{h} \in\left(\mathcal{P}^{p_{i}}\left(K_{i}\right)\right)^{d}, d=1,2$. Then for $r \geq 2$, there exists a constant $C_{I}>0$ such that

$$
\left\|\boldsymbol{v}_{h}\right\|_{L^{r}\left(K_{i}\right)^{d}} \leq C_{I} h_{i}^{\left(\frac{2}{r}-1\right)}\left\|\boldsymbol{v}_{h}\right\|_{L^{2}\left(K_{i}\right)^{d}} .
$$
$\mathbf{L}_{h}$.

In the following lemma, we state some approximation properties of $\mathbf{L}^{2}$-projection

Lemma $2.4\left(\mathbf{L}^{2}\right.$-projection $\left.\mathbf{L}_{h}\right)$. Let $\boldsymbol{\psi} \in \mathbf{H}^{s}\left(K_{i}\right)$ and $\boldsymbol{\psi}_{h}=\mathbf{L}_{h} \boldsymbol{\psi} \in \mathcal{P}^{k}\left(K_{i}\right)$ be the $\mathbf{L}^{2}$-projection of $\boldsymbol{\psi}$ onto $\mathcal{P}^{k}\left(K_{i}\right)$. Then, the following approximation properties hold:

$$
\left\|\boldsymbol{\psi}-\boldsymbol{\psi}_{h}\right\|_{\mathbf{L}^{2}\left(K_{i}\right)}+h_{i}^{\frac{1}{2}}\left\|\boldsymbol{\psi}-\boldsymbol{\psi}_{h}\right\|_{\mathbf{L}^{2}\left(\partial K_{i}\right)} \leq C h_{i}^{\mu}\|\boldsymbol{\psi}\|_{\mathbf{H}^{s}\left(K_{i}\right)}
$$

and

$$
\left\|\boldsymbol{\psi}-\boldsymbol{\psi}_{h}\right\|_{\mathbf{L}^{4}\left(K_{i}\right)} \leq C h_{i}^{\mu-\frac{1}{2}}\|\boldsymbol{\psi}\|_{\mathbf{H}^{s}\left(K_{i}\right)},
$$

where $\mu=\min \{s, k+1\}$.

Proof. The first inequality of the lemma follows from Lemma 2.1 and the trace inequality (2.2). For the estimate of $\left\|\boldsymbol{\psi}-\boldsymbol{\psi}_{h}\right\|_{\mathbf{L}^{4}\left(K_{i}\right)}$, we use inverse inequality (2.3). This completes the proof. 


\section{Nonselfadjoint linear Elliptic PROBlems}

For our error analysis of discontinuous Galerkin methods applied to nonlinear elliptic problems (1.1), we need some results on the corresponding linearized problem. Since the linearized problem is a nonselfadjoint elliptic problem, in this section, we consider the following second order linear nonselfadjoint elliptic boundary value problem of the form:

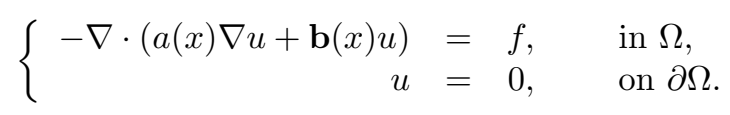

We adopt the following assumptions on the problem (3.1):

(i) There exists $\alpha_{0}>0$ such that $0<\alpha_{0} \leq a(x)$ for all $x \in \bar{\Omega}$.

(ii) $a$ and $\mathbf{b}$ are assumed to be smooth with $M=\max \left\{\|a\|_{L^{\infty}(\Omega)},\|\mathbf{b}\|_{\mathbf{L}^{\infty}(\Omega)}\right\}$ and $f \in L^{2}(\Omega)$.

(iii) There exists a unique solution $u \in H^{2}(\Omega) \cap H_{0}^{1}(\Omega)$ to the problem (3.1) satisfying

$$
\|u\|_{H^{2}(\Omega)} \leq C\|f\|_{L^{2}(\Omega)} .
$$

3.1. DG Methods. Now, we introduce new variables $\mathbf{q}=\nabla u, \boldsymbol{\sigma}=a \mathbf{q}+\mathbf{b} u$ and rewrite (3.1) as

$$
\begin{aligned}
\mathbf{q} & =\nabla u, & & \text { in } \Omega, \\
\boldsymbol{\sigma} & =a \mathbf{q}+\mathbf{b} u, & & \text { in } \Omega, \\
-\nabla \cdot \boldsymbol{\sigma} & =f, & & \text { in } \Omega, \\
u & =0, & & \text { on } \partial \Omega .
\end{aligned}
$$

Then, we arrive at DG methods corresponding to the formulation (3.2): find $\left(u_{h}, \mathbf{q}_{h}, \boldsymbol{\sigma}_{h}\right) \in V_{h} \times \mathbf{W}_{h} \times \mathbf{W}_{h}$ satisfying

$$
\begin{aligned}
\left(\mathbf{q}_{h}, \mathbf{w}_{h}\right)+\left(u_{h}, \nabla \cdot \mathbf{w}_{h}\right)-\left\langle\hat{u}_{h}, \mathbf{w}_{h} \cdot \boldsymbol{\nu}\right\rangle & =0, & & \forall \mathbf{w}_{h} \in \mathbf{W}_{h}, \\
\left(a \mathbf{q}_{h}, \boldsymbol{\tau}_{h}\right)+\left(\mathbf{b} u_{h}, \boldsymbol{\tau}_{h}\right)-\left(\boldsymbol{\sigma}_{h}, \boldsymbol{\tau}_{h}\right) & =0, & & \forall \boldsymbol{\tau}_{h} \in \mathbf{W}_{h}, \\
\left(\boldsymbol{\sigma}_{h}, \nabla v_{h}\right)-\left\langle\hat{\boldsymbol{\sigma}}_{h} \cdot \boldsymbol{\nu}, v_{h}\right\rangle & =\left(f, v_{h}\right), & & \forall v_{h} \in V_{h},
\end{aligned}
$$

where the numerical fluxes $\hat{u}_{h}$ and $\hat{\boldsymbol{\sigma}}_{h}$ have to be suitably chosen in order to ensure the stability and to enhance the accuracy of the method. As in the linear elliptic case [9], we define numerical fluxes $\hat{u}_{h}$ and $\hat{\boldsymbol{\sigma}}_{h}$ on each $e_{k} \in \Gamma_{I}$ as:

$$
\begin{aligned}
\hat{u}_{h}\left(u_{h}, \boldsymbol{\sigma}_{h}\right) & =\left\{\left\{u_{h}\right\}\right\}+\mathbf{C}_{12} \cdot \llbracket u_{h} \rrbracket-C_{22} \llbracket \boldsymbol{\sigma}_{h} \rrbracket, \\
\hat{\boldsymbol{\sigma}}_{h}\left(u_{h}, \boldsymbol{\sigma}_{h}\right) & =\left\{\left\{\boldsymbol{\sigma}_{h} \rrbracket-C_{11} \llbracket u_{h} \rrbracket-\mathbf{C}_{12} \llbracket \boldsymbol{\sigma}_{h} \rrbracket,\right.\right.
\end{aligned}
$$

and if $e_{k} \in \Gamma_{\partial}$, i.e., $e_{k}=\partial K \cap \partial \Omega$ for some $K \in \mathcal{T}_{h}$, then we denote numerical fluxes by

$$
\begin{aligned}
\hat{u}_{h} & =0, \\
\hat{\boldsymbol{\sigma}}_{h} & =\boldsymbol{\sigma}_{\left.h\right|_{K}}-C_{11} u_{\left.h\right|_{K}} \boldsymbol{\nu}_{K},
\end{aligned}
$$

where the parameters $C_{11}, \mathbf{C}_{12} \in \mathbb{R}^{2}$ and $C_{22}$ are single valued and are chosen so that the following conditions are satisfied:

$$
\begin{array}{cc}
\text { (i) } & \left|\mathbf{C}_{12}\right| \leq C, \\
\text { (ii) } & \bar{C}_{22} \sim \frac{C_{22}}{\bar{C}_{11}} \sim \frac{1}{C_{11}} \sim \frac{1}{\underline{C}_{11}}, \\
\text { (iii) } &
\end{array}
$$


for some constant $C$, where

$$
\bar{C}_{22}=\max \left\{C_{22}(x): x \in \Gamma\right\}, \quad \underline{C}_{22}=\min \left\{C_{22}(x): x \in \Gamma\right\},
$$

(3.7) and

$$
\bar{C}_{11}=\max \left\{C_{11}(x): x \in \Gamma\right\}, \quad \underline{C}_{11}=\min \left\{C_{11}(x): x \in \Gamma\right\} .
$$

When $C_{11}$ is decreased and $C_{22}$ is increased, we obtain better order of convergence. In [9], the authors have shown that $u_{h}$ and $\mathbf{q}_{h}$ converge in $L^{2}$-norm with order $k$ and $k+\frac{1}{2}$, respectively, for any $k \geq 0$ provided $C_{11}$ and $C_{22}$ are of order one. These DG methods have not been paid much attention since they can be very difficult to implement when $C_{22} \neq 0$.

In the recent past, local DG-hybridizable (LDG-H) methods are introduced in [14, which are characterized by

$$
\begin{aligned}
& \hat{u}_{h}=\left(\frac{\tau^{+}}{\tau^{-}+\tau^{+}}\right) u_{h}^{+}+\left(\frac{\tau^{-}}{\tau^{-}+\tau^{+}}\right) u_{h}^{-}-\left(\frac{1}{\tau^{-}+\tau^{+}}\right) \llbracket \boldsymbol{\sigma}_{h} \rrbracket, \\
& \hat{\boldsymbol{\sigma}}_{h}=\left(\frac{\tau^{-}}{\tau^{-}+\tau^{+}}\right) \boldsymbol{\sigma}_{h}^{+}+\left(\frac{\tau^{+}}{\tau^{-}+\tau^{+}}\right) \boldsymbol{\sigma}_{h}^{-}-\left(\frac{\tau^{+} \tau^{-}}{\tau^{-}+\tau^{+}}\right) \llbracket u_{h} \rrbracket,
\end{aligned}
$$

where $\tau^{ \pm}$are nonnegative constants. Although $C_{22}=1 /\left(\tau^{-}+\tau^{+}\right) \neq 0$, these methods can be implemented efficiently; see [14. In [15], authors have proved optimal convergence in flux with the choice (3.8a)-(3.8b).

The numerical fluxes are conservative (cf. 3]), since they are single valued on $e_{k} \in \Gamma_{I}$, that is, on $e_{k} \in \Gamma_{I}$,

$$
\llbracket \hat{u}_{h} \rrbracket=0, \quad \llbracket \hat{\boldsymbol{\sigma}}_{h} \rrbracket=0,
$$

and consistent (cf. [3]), since for continuous functions $u$ and $\boldsymbol{\sigma}$, the following conditions hold:

$$
\hat{u}_{h}(u, \boldsymbol{\sigma})=u, \quad \hat{\boldsymbol{\sigma}}_{h}(u, \boldsymbol{\sigma})=\boldsymbol{\sigma} .
$$

This completes the definition of DG methods.

We, now, introduce some notation and definitions for our subsequent use. Given a function $\mathbf{w} \in \mathbf{H}\left(\operatorname{div}, \mathcal{T}_{h}\right)$, where $\mathbf{H}\left(\operatorname{div}, \mathcal{T}_{h}\right)$ is the broken $\mathbf{H}(\operatorname{div})$-space and an arbitrary element $K \in \mathcal{T}_{h}$, the Raviart-Thomas projection $\Pi_{l}^{R T} \mathbf{w}_{\left.\right|_{K}} \in \mathcal{P}^{l}(K)+$ $\boldsymbol{x} \mathcal{P}^{l}(K)$ is defined by

(3.11a) $\left\langle\left(\Pi_{l}^{R T} \mathbf{w}-\mathbf{w}\right) \cdot \boldsymbol{\nu}, \mu\right\rangle_{e}=0 \quad \forall \mu \in \mathcal{P}^{l}(e)$ for all edges $e$ of $K$,

$$
\left(\Pi_{l}^{R T} \mathbf{w}-\mathbf{w}, \boldsymbol{\tau}\right)_{K}=0 \quad \forall \boldsymbol{\tau} \in \mathcal{P}^{l-1}(K) .
$$

Given a function $\eta \in L^{2}(\Omega)$, and an arbitrary element $K \in \mathcal{T}_{h}$ with $e$ as one of its edges, we define the restriction of $P_{\partial}^{l} \eta_{\left.\right|_{e}} \in \mathcal{P}^{l}(e)$ as

$$
\left\langle P_{\partial}^{l} \eta-\eta, \mu\right\rangle_{e}=0, \quad \forall \mu \in \mathcal{P}^{l}(e) .
$$

Note that on the interior faces, $P_{\partial}^{l} \eta$ is, in general, double-valued.

Given a function $\zeta \in L^{2}(\Omega)$ and an arbitrary simplex $K \in \mathcal{T}_{h}$, the restriction of $P^{l} \zeta$ to $K$ is defined as the element of $\mathcal{P}^{l}(K)$ that satisfies

$$
\left(P^{l} \zeta-\zeta, v\right)=0, \quad \forall v \in \mathcal{P}^{l}(K) .
$$

For our future use, for any $\mathbf{w} \in \mathbf{L}^{2}(\Gamma)$ we set

$$
|\mathbf{w}|_{L^{2}(\Gamma ; h)}=\left(\sum_{i=1}^{N_{h}} h_{i}\|\mathbf{w} \cdot \boldsymbol{\nu}\|_{L^{2}\left(\partial K_{i}\right)}^{2}\right)^{\frac{1}{2}} .
$$


3.2. Existence and uniqueness of the discrete solution. Since $V_{h}$ and $\mathbf{W}_{h}$ are finite dimensional spaces, the system (3.3a)-(3.3c) leads to a system of linear algebraic equations. Therefore, for complete solvability of (3.3a)-(3.3c), it is sufficient to show uniqueness. Thus, for $f=0$, it is enough to show that $u_{h}=0, \mathbf{q}_{h}=0$ and $\boldsymbol{\sigma}_{h}=0$. Now, when $f=0$, the system (3.3a)-(3.3c) reduces to

$$
\begin{aligned}
\left(\mathbf{q}_{h}, \mathbf{w}_{h}\right)+\left(u_{h}, \nabla \cdot \mathbf{w}_{h}\right)-\left\langle\hat{u}_{h}, \mathbf{w}_{h} \cdot \boldsymbol{\nu}\right\rangle & =0, \quad \forall \mathbf{w}_{h} \in \mathbf{W}_{h}, \\
\left(a \mathbf{q}_{h}, \boldsymbol{\tau}_{h}\right)+\left(\mathbf{b} u_{h}, \boldsymbol{\tau}_{h}\right)-\left(\boldsymbol{\sigma}_{h}, \boldsymbol{\tau}_{h}\right) & =0, \quad \forall \boldsymbol{\tau}_{h} \in \mathbf{W}_{h}, \\
\left(\boldsymbol{\sigma}_{h}, \nabla v_{h}\right)-\left\langle\hat{\boldsymbol{\sigma}}_{h} \cdot \boldsymbol{\nu}, v_{h}\right\rangle & =0, \quad \forall v_{h} \in V_{h} .
\end{aligned}
$$

Substitute $\tau_{h}=\sigma_{h}$ in (3.14b) to obtain

$$
\left\|\boldsymbol{\sigma}_{h}\right\| \leq C\left(\left\|\mathbf{q}_{h}\right\|+\left\|u_{h}\right\|\right) .
$$

Now, substitute $\mathbf{w}_{h}=\boldsymbol{\sigma}_{h}$ in (3.14a), $\boldsymbol{\tau}_{h}=\mathbf{q}_{h}$ in (3.14b) and $v_{h}=u_{h}$ in (3.14c) to obtain

$$
\left(a \mathbf{q}_{h}, \mathbf{q}_{h}\right)+\left\langle u_{h}, \boldsymbol{\sigma}_{h} \cdot \boldsymbol{\nu}\right\rangle-\left\langle\hat{u}_{h}, \boldsymbol{\sigma}_{h} \cdot \boldsymbol{\nu}\right\rangle-\left\langle\hat{\boldsymbol{\sigma}}_{h} \cdot \boldsymbol{\nu}, u_{h}\right\rangle+\left(\mathbf{b} u_{h}, \mathbf{q}_{h}\right)=0
$$

Use the definition of numerical fluxes, $0<\alpha_{0} \leq a$ along with Young's inequality to arrive at

$$
\left\|\mathbf{q}_{h}\right\|^{2}+\int_{\Gamma} C_{11} \llbracket u_{h} \rrbracket^{2} d s+\int_{\Gamma_{I}} C_{22} \llbracket \boldsymbol{\sigma}_{h} \rrbracket^{2} d s \leq C\left\|u_{h}\right\|^{2}
$$

From (3.15) and (3.16), we can complete the proof of uniqueness, provided $u_{h}=0$. Now, we consider the dual problem:

$$
\begin{aligned}
\mathbf{p} & =\nabla \phi, & & \text { in } \Omega, \\
-\boldsymbol{\chi} & =a \mathbf{p}, & & \text { in } \Omega, \\
\nabla \cdot \boldsymbol{\chi}+\mathbf{b} \cdot \mathbf{p} & =\lambda, & & \text { in } \Omega, \\
\phi & =0, & & \text { on } \partial \Omega,
\end{aligned}
$$

which satisfies the elliptic regularity:

$$
\|\phi\|_{H^{2}(\Omega)}+\|\chi\|_{\mathbf{H}^{1}(\Omega)}+\|\mathbf{p}\|_{\mathbf{H}^{1}(\Omega)} \leq C\|\lambda\| .
$$

Multiply (3.17c) by $u_{h},\left(3.17 \mathrm{~b}\right.$ ) by $\mathbf{q}_{h}$ and (3.17a by $\boldsymbol{\sigma}_{h}$. Then integrate and add them to arrive at

$$
\left(u_{h}, \lambda\right)=\left(\nabla \cdot \boldsymbol{\chi}, u_{h}\right)+\left(\mathbf{b} \cdot \mathbf{p}, u_{h}\right)+\left(\boldsymbol{\chi}, \mathbf{q}_{h}\right)+\left(a \mathbf{p}, \mathbf{q}_{h}\right)-\left(\mathbf{p}, \boldsymbol{\sigma}_{h}\right)+\left(\nabla \phi, \boldsymbol{\sigma}_{h}\right) .
$$

Now using the system (3.14a) $-(3.14 \mathrm{c})$ and the definition of projection $P^{k}$, we obtain

$$
\begin{aligned}
\left(u_{h}, \lambda\right)= & \left(\nabla \cdot\left(\boldsymbol{\chi}-P^{k} \boldsymbol{\chi}\right), u_{h}\right)+\left(\mathbf{b} \cdot\left(\mathbf{p}-P^{k} \mathbf{p}\right), u_{h}\right)+\left(\boldsymbol{\chi}-P^{k} \boldsymbol{\chi}, \mathbf{q}_{h}\right) \\
& +\left(a\left(\mathbf{p}-P^{k} \mathbf{p}\right), \mathbf{q}_{h}\right)-\left(\mathbf{p}-P^{k} \mathbf{p}, \boldsymbol{\sigma}_{h}\right)+\left(\nabla\left(\phi-P^{k} \phi\right), \boldsymbol{\sigma}_{h}\right) \\
& +\left\langle\hat{u}_{h}, P^{k} \boldsymbol{\chi} \cdot \boldsymbol{\nu}\right\rangle+\left\langle\hat{\boldsymbol{\sigma}}_{h} \cdot \boldsymbol{\nu}, P^{k} \phi\right\rangle .
\end{aligned}
$$


Since $\llbracket \phi \rrbracket=0$ and $\llbracket \chi \rrbracket=0$ on interior edges and $\phi=0$ and $\hat{u}_{h}=0$ on boundary edges, we easily arrive at

$$
\begin{aligned}
\left(u_{h}, \lambda\right)= & \left(\nabla \cdot\left(\boldsymbol{\chi}-P^{k} \boldsymbol{\chi}\right), u_{h}\right)+\left(\mathbf{b} \cdot\left(\mathbf{p}-P^{k} \mathbf{p}\right), u_{h}\right)+\left(\boldsymbol{\chi}-P^{k} \boldsymbol{\chi}, \mathbf{q}_{h}\right) \\
& +\left(a\left(\mathbf{p}-P^{k} \mathbf{p}\right), \mathbf{q}_{h}\right)-\left(\mathbf{p}-P^{k} \mathbf{p}, \boldsymbol{\sigma}_{h}\right)+\left(\nabla\left(\phi-P^{k} \phi\right), \boldsymbol{\sigma}_{h}\right) \\
& -\left\langle\hat{u}_{h},\left(\boldsymbol{\chi}-P^{k} \boldsymbol{\chi}\right) \cdot \boldsymbol{\nu}\right\rangle-\left\langle\hat{\boldsymbol{\sigma}}_{h} \cdot \boldsymbol{\nu}, \phi-P^{k} \phi\right\rangle \\
= & -\left(\boldsymbol{\chi}-P^{k} \boldsymbol{\chi}, \nabla u_{h}\right)+\left\langle u_{h}-\hat{u}_{h},\left(\boldsymbol{\chi}-P^{k} \boldsymbol{\chi}\right) \cdot \boldsymbol{\nu}\right\rangle+\left(\mathbf{b} \cdot\left(\mathbf{p}-P^{k} \mathbf{p}, u_{h}\right)\right. \\
& +\left(a\left(\mathbf{p}-P^{k} \mathbf{p}\right), \mathbf{q}_{h}\right)+\left(\phi-P^{k} \phi, \nabla \cdot \boldsymbol{\sigma}_{h}\right)+\left\langle\left(\boldsymbol{\sigma}_{h}-\hat{\boldsymbol{\sigma}}_{h}\right) \cdot \boldsymbol{\nu}, \phi-P^{k} \phi\right\rangle \\
= & \left\langle u_{h}-\hat{u}_{h},\left(\boldsymbol{\chi}-P^{k} \boldsymbol{\chi}\right) \cdot \boldsymbol{\nu}\right\rangle+\left(\mathbf{b} \cdot\left(\mathbf{p}-P^{k} \mathbf{p}, u_{h}\right)+\left(a\left(\mathbf{p}-P^{k} \mathbf{p}\right), \mathbf{q}_{h}\right)\right. \\
& +\left\langle\left(\boldsymbol{\sigma}_{h}-\hat{\boldsymbol{\sigma}}_{h}\right) \cdot \boldsymbol{\nu}, \phi-P^{k} \phi\right\rangle .
\end{aligned}
$$

Using the properties of the projection, definition of numerical fluxes, (3.7) and regularity result (3.18), we obtain

$$
\begin{aligned}
&\left\|u_{h}\right\| \leq C h^{\frac{1}{2}}\left(\max \left\{\frac{1}{C_{11}}, \bar{C}_{22}, h^{2} \bar{C}_{11}, \frac{h^{2}}{\underline{C}_{22}}\right\}\left\{\int_{\Gamma} C_{11} \llbracket u_{h} \rrbracket^{2} d s+\int_{\Gamma_{I}} C_{22} \llbracket \boldsymbol{\sigma}_{h} \rrbracket^{2} d s\right\}\right)^{\frac{1}{2}} \\
&(3.21) \quad+C h\left(\left\|u_{h}\right\|+\left\|\mathbf{q}_{h}\right\|\right) .
\end{aligned}
$$

An application of (3.16) yields

$$
\left\|u_{h}\right\| \leq C h^{\frac{1}{2}}\left(\max \left\{h, \frac{1}{\underline{C_{11}}}, \bar{C}_{22}, h^{2} \bar{C}_{11}, \frac{h^{2}}{\underline{C}_{22}}\right\}\right)^{\frac{1}{2}}\left\|u_{h}\right\| .
$$

By the definitions of parameters $C_{11}$ and $C_{22}$, we can choose $h$ sufficiently small such that $1-C h^{\frac{1}{2}}\left(\max \left\{h, \frac{1}{\underline{C}_{11}}, \bar{C}_{22}, h^{2} \bar{C}_{11}, \frac{h^{2}}{\underline{C}_{22}}\right\}\right)^{\frac{1}{2}}>0$ and this yields $\left\|u_{h}\right\|=0$, that is, $u_{h}=0$ on each $K \in \mathcal{T}_{h}$. On substituting this in (3.16) we observe that $\mathbf{q}_{h}=0$ on each $K \in \mathcal{T}_{h}$ with $\llbracket u_{h} \rrbracket=0$ and $\llbracket \boldsymbol{\sigma}_{h} \rrbracket=0$ on each interior edge and finally, by (3.15), we conclude that $\boldsymbol{\sigma}_{h}=0$. This shows that for homogeneous data, the system (3.14a)-(3.14c) has only zero solution and hence, the system (3.3a)-(3.3c) has a unique solution. This also ensures existence of the solution of the system (3.3a) $-3.3 \mathrm{c}$.

Now, on each element $K \in \mathcal{T}_{h}$, we define the function $\boldsymbol{\sigma}_{h}^{*}$ as the only element of $\mathcal{P}^{k}(K)+\boldsymbol{x} \mathcal{P}^{k}(K)$ satisfying

(3.23a) $\left\langle\left(\boldsymbol{\sigma}_{h}^{*}-\hat{\boldsymbol{\sigma}}_{h}\right) \cdot \boldsymbol{\nu}, \mu\right\rangle_{e}=0, \quad \forall \mu \in \mathcal{P}^{k}(e)$ for all edges $e$ of $K$,

$$
\left(\boldsymbol{\sigma}_{h}^{*}-\boldsymbol{\sigma}_{h}, \mathbf{w}\right)_{K}=0, \quad \forall \mathbf{w} \in \mathcal{P}^{k-1}(K) \text {. }
$$

3.3. Some properties of $\sigma_{h}^{*}$. Below, we state, without proof, the lemma giving some properties of $\boldsymbol{\sigma}_{h}^{*}$. For a proof, we refer to [12, Lemma 4.1]

Lemma 3.1. If $\boldsymbol{\sigma}_{h}^{*}$ is defined as in (3.23), then it satisfies the following properties:

(i) $\boldsymbol{\sigma}_{h}^{*}-\Pi_{k}^{R T} \boldsymbol{\sigma} \in \mathbf{H}\left(\operatorname{div}, \mathcal{T}_{h}\right)$,

(ii) $\nabla \cdot\left(\boldsymbol{\sigma}_{h}^{*}-\Pi_{k}^{R T} \boldsymbol{\sigma}\right)=0$ in $\Omega$,

(iii) $\boldsymbol{\sigma}_{h}^{*}-\Pi_{k}^{R T} \boldsymbol{\sigma} \in \mathbf{W}_{h}$.

We are now ready to state an estimate of $\boldsymbol{\sigma}_{h}^{*}-\Pi_{k}^{R T} \boldsymbol{\sigma}$, whose proof can be found in [12, Lemma 4.2].

Lemma 3.2. If $\boldsymbol{\sigma}_{h}^{*}$ and $\Pi_{k}^{R T}$ are defined by (3.23) and (3.11), respectively, then there exists a positive constant $C$ independent of $h_{K}$ such that for each $K \in \mathcal{T}_{h}$ the 
following estimate holds:

$$
\left\|\boldsymbol{\sigma}_{h}^{*}-\Pi_{k}^{R T} \boldsymbol{\sigma}_{h}\right\|_{L^{2}(K)} \leq C h_{K}^{\frac{1}{2}}\left\|P_{\partial}^{k}\left(\hat{\boldsymbol{\sigma}}_{h}-\boldsymbol{\sigma}_{h}\right) \cdot \boldsymbol{\nu}\right\|_{L^{2}(\partial K)} .
$$

The main properties of this new approximation $\boldsymbol{\sigma}_{h}^{*}$ are stated in the following theorem.

Theorem 3.1. For any method of the form (3.3a)-(3.3c) and for $\boldsymbol{\sigma}_{h}^{*}$ defined as in (3.23), we obtain the following results:

$$
\begin{array}{r}
\boldsymbol{\sigma}_{h}^{*} \in \mathbf{H}\left(\operatorname{div}, \mathcal{T}_{h}\right), \\
\left\|\nabla \cdot\left(\boldsymbol{\sigma}-\boldsymbol{\sigma}_{h}^{*}\right)\right\| \leq C\left\|f-P^{k} f\right\| .
\end{array}
$$

Proof. We use (i) of Lemma 3.1 and the fact that $\Pi_{k}^{R T} \boldsymbol{\sigma}$ is in $\mathbf{H}\left(\operatorname{div} ; \mathcal{T}_{h}\right)$ to obtain $\boldsymbol{\sigma}_{h}^{*} \in \mathbf{H}\left(\operatorname{div} ; \mathcal{T}_{h}\right)$. To estimate $\left\|\nabla \cdot\left(\boldsymbol{\sigma}-\boldsymbol{\sigma}_{h}^{*}\right)\right\|$, we use (ii) of Lemma 3.1 and the well-known property of projection $\Pi_{k}^{R T}$; see, for example, [6], to find that

$$
\begin{aligned}
\left\|\nabla \cdot\left(\boldsymbol{\sigma}-\boldsymbol{\sigma}_{h}^{*}\right)\right\| & =\left\|\nabla \cdot\left(\boldsymbol{\sigma}-\Pi_{k}^{R T} \boldsymbol{\sigma}\right)\right\| \\
& =\left\|f-P^{k} f\right\| .
\end{aligned}
$$

This completes the rest of the proof.

For our future use, we set $e_{u}=u-u_{h}, \mathbf{e}_{\mathbf{q}}=\mathbf{q}-\mathbf{q}_{h}$ and $\mathbf{e}_{\boldsymbol{\sigma}}=\boldsymbol{\sigma}-\boldsymbol{\sigma}_{h}$.

3.4. Optimal convergence of $\sigma_{h}$ and $\mathbf{q}_{h}$. Since the numerical fluxes $\hat{u}_{h}$ and $\hat{\boldsymbol{\sigma}}_{h}$ are consistent, we easily obtain the following system of equations:

$$
\begin{aligned}
\left(\mathbf{q}-\mathbf{q}_{h}, \mathbf{w}_{h}\right)+\left(u-u_{h}, \nabla \cdot \mathbf{w}_{h}\right)-\left\langle u-\hat{u}_{h}, \mathbf{w}_{h} \cdot \boldsymbol{\nu}\right\rangle & =0, \quad \forall \mathbf{w}_{h} \in \mathbf{W}_{h}, \\
\left(a\left(\mathbf{q}-\mathbf{q}_{h}\right), \boldsymbol{\tau}_{h}\right)+\left(\mathbf{b}\left(u-u_{h}\right), \boldsymbol{\tau}_{h}\right)-\left(\boldsymbol{\sigma}-\boldsymbol{\sigma}_{h}, \boldsymbol{\tau}_{h}\right) & =0, \quad \forall \boldsymbol{\tau}_{h} \in \mathbf{W}_{h}, \\
\left(\boldsymbol{\sigma}-\boldsymbol{\sigma}_{h}, \nabla v_{h}\right)-\left\langle\left(\boldsymbol{\sigma}-\hat{\boldsymbol{\sigma}}_{h}\right) \cdot \boldsymbol{\nu}, v_{h}\right\rangle & =0, \quad \forall v_{h} \in V_{h} .
\end{aligned}
$$

Lemma 3.3. For any method of the form (3.3a)-(3.3c), there exists a positive constant $C$ such that the following estimate holds:

$$
\left\|\boldsymbol{\sigma}-\boldsymbol{\sigma}_{h}\right\| \leq C\left(\left\|\boldsymbol{\sigma}-\mathbf{L}_{h} \boldsymbol{\sigma}\right\|+\left\|\mathbf{q}-\mathbf{q}_{h}\right\|+\left\|u-u_{h}\right\|\right),
$$

where $\mathbf{L}_{h}$ is the $\mathbf{L}^{2}$-projection as in Lemma 2.4.

Proof. Substitute $\boldsymbol{\tau}_{h}=\mathbf{L}_{h} \boldsymbol{\sigma}-\boldsymbol{\sigma}_{h}$ in (3.26b) to obtain

$$
\left(\mathbf{e}_{\boldsymbol{\sigma}}, \mathbf{L}_{h} \boldsymbol{\sigma}-\boldsymbol{\sigma}_{h}\right)=\left(a \mathbf{e}_{\mathbf{q}}, \mathbf{L}_{h} \boldsymbol{\sigma}-\boldsymbol{\sigma}_{h}\right)+\left(\mathbf{b} e_{u}, \mathbf{L}_{h} \boldsymbol{\sigma}-\boldsymbol{\sigma}_{h}\right) .
$$

Note that using the property of $\mathbf{L}^{2}$-projection $\mathbf{L}_{h}$ in Lemma 2.4, we get

$$
\left(\mathbf{e}_{\boldsymbol{\sigma}}, \mathbf{L}_{h} \boldsymbol{\sigma}-\boldsymbol{\sigma}_{h}\right)=\left\|\mathbf{L}_{h} \boldsymbol{\sigma}-\boldsymbol{\sigma}_{h}\right\|^{2} .
$$

Using (3.29) in (3.28), we arrive at

$$
\begin{aligned}
\left\|\mathbf{L}_{h} \boldsymbol{\sigma}-\boldsymbol{\sigma}_{h}\right\|^{2} & =\left(a \mathbf{e}_{\mathbf{q}}, \mathbf{L}_{h} \boldsymbol{\sigma}-\boldsymbol{\sigma}_{h}\right)+\left(\mathbf{b} e_{u}, \mathbf{L}_{h} \boldsymbol{\sigma}-\boldsymbol{\sigma}_{h}\right) \\
& \leq C\left(\left\|\mathbf{e}_{\mathbf{q}}\right\|+\left\|e_{u}\right\|\right)\left\|\mathbf{L}_{h} \boldsymbol{\sigma}-\boldsymbol{\sigma}_{h}\right\|,
\end{aligned}
$$

and hence,

$$
\left\|\mathbf{L}_{h} \boldsymbol{\sigma}-\boldsymbol{\sigma}_{h}\right\| \leq C\left(\left\|\mathbf{e}_{\mathbf{q}}\right\|+\left\|e_{u}\right\|\right) .
$$

Now, using triangle inequality, we obtain

$$
\left\|\mathbf{e}_{\boldsymbol{\sigma}}\right\| \leq\left\|\boldsymbol{\sigma}-\mathbf{L}_{h} \boldsymbol{\sigma}\right\|+C\left(\left\|\mathbf{e}_{\mathbf{q}}\right\|+\left\|e_{u}\right\|\right),
$$

and this completes the rest of the proof. 
Lemma 3.4. For any method of the form (3.3a)-(3.3c), there exists a positive constant $C$ such that

$$
\left\|\mathbf{q}-\mathbf{q}_{h}\right\| \leq C\left(\left\|\mathbf{q}-I_{h} \mathbf{q}\right\|+\left\|\boldsymbol{\sigma}-\mathbf{L}_{h} \boldsymbol{\sigma}\right\|+\left\|\boldsymbol{\sigma}-\Pi_{k}^{R T} \boldsymbol{\sigma}\right\|+\left\|u-u_{h}\right\|+\Theta_{k}\right)
$$

holds, where $I_{h}$ is defined as in (2.1), $\Pi_{k}^{R T}$ is defined as in (3.11) and $\Theta_{k}$ is defined by

$$
\Theta_{k}:=\left\|\boldsymbol{\sigma}_{h}-\Pi_{k}^{R T} \boldsymbol{\sigma}_{h}\right\|+\left|P_{\partial}^{k}\left(\hat{\boldsymbol{\sigma}}_{h}-\boldsymbol{\sigma}_{h}\right)\right|_{L^{2}(\Gamma ; h)} .
$$

Proof. We write

$$
\left(a \mathbf{e}_{\mathbf{q}}, \mathbf{e}_{\mathbf{q}}\right)=\left(a \mathbf{e}_{\mathbf{q}}, \mathbf{q}-I_{h} \mathbf{q}\right)+\left(a \mathbf{e}_{\mathbf{q}}, I_{h} \mathbf{q}-\mathbf{q}_{h}\right) .
$$

Using (3.26b), we obtain

$$
\begin{aligned}
\left(a \mathbf{e}_{\mathbf{q}}, \mathbf{e}_{\mathbf{q}}\right) & =\left(a \mathbf{e}_{\mathbf{q}}, \mathbf{q}-I_{h} \mathbf{q}\right)+\left(\mathbf{e}_{\boldsymbol{\sigma}}, I_{h} \mathbf{q}-\mathbf{q}_{h}\right)-\left(\mathbf{b} e_{u}, I_{h} \mathbf{q}-\mathbf{q}_{h}\right) \\
& =\left(a \mathbf{e}_{\mathbf{q}}, \mathbf{q}-I_{h} \mathbf{q}\right)+\left(\mathbf{e}_{\boldsymbol{\sigma}}, \mathbf{e}_{\mathbf{q}}\right)-\left(\mathbf{e}_{\boldsymbol{\sigma}}, \mathbf{q}-I_{h} \mathbf{q}\right)-\left(\mathbf{b} e_{u}, I_{h} \mathbf{q}-\mathbf{q}_{h}\right) .
\end{aligned}
$$

Now, using the definitions of $\boldsymbol{\sigma}_{h}^{*}$ and $\Pi_{k}^{R T} \boldsymbol{\sigma}$, we rewrite the second term on the right-hand side of (3.32) as

$$
\left(\mathbf{e}_{\boldsymbol{\sigma}}, \mathbf{e}_{\mathbf{q}}\right)=\left(\mathbf{e}_{\mathbf{q}}, \boldsymbol{\sigma}-\Pi_{k}^{R T} \boldsymbol{\sigma}\right)+\left(\mathbf{e}_{\mathbf{q}}, \Pi_{k}^{R T} \boldsymbol{\sigma}-\boldsymbol{\sigma}_{h}^{*}\right)+\left(\mathbf{e}_{\mathbf{q}}, \boldsymbol{\sigma}_{h}^{*}-\boldsymbol{\sigma}_{h}\right) .
$$

Note that $\nabla \cdot\left(\Pi_{k}^{R T} \boldsymbol{\sigma}-\boldsymbol{\sigma}_{h}^{*}\right)=0$, by property (ii) of Lemma 3.1 and use (3.26a) to obtain

$$
\left(\mathbf{e}_{\mathbf{q}}, \Pi_{k}^{R T} \boldsymbol{\sigma}-\boldsymbol{\sigma}_{h}^{*}\right)=\left\langle u-\hat{u}_{h},\left(\Pi_{k}^{R T} \boldsymbol{\sigma}-\boldsymbol{\sigma}_{h}^{*}\right) \cdot \boldsymbol{\nu}\right\rangle .
$$

Since $\Pi_{k}^{R T} \boldsymbol{\sigma}-\boldsymbol{\sigma}_{h}^{*} \in \mathbf{H}\left(\operatorname{div}, \mathcal{T}_{h}\right)$, by property (iii) of Lemma 3.1 and $u-\hat{u}_{h}=0$ on $\partial \Omega$, we arrive at

$$
\left(\mathbf{e}_{\mathbf{q}}, \Pi_{k}^{R T} \boldsymbol{\sigma}-\boldsymbol{\sigma}_{h}^{*}\right)=0
$$

Hence,

$$
\left(\mathbf{e}_{\boldsymbol{\sigma}}, \mathbf{e}_{\mathbf{q}}\right)=\left(\mathbf{e}_{\mathbf{q}}, \boldsymbol{\sigma}-\Pi_{k}^{R T} \boldsymbol{\sigma}\right)+\left(\mathbf{e}_{\mathbf{q}}, \boldsymbol{\sigma}_{h}^{*}-\boldsymbol{\sigma}_{h}\right) .
$$

Thus, substituting (3.34) in (3.32), we rewrite the resulting equation as

$$
\begin{aligned}
\left(a \mathbf{e}_{\mathbf{q}}, \mathbf{e}_{\mathbf{q}}\right)= & \left(a \mathbf{e}_{\mathbf{q}}, \mathbf{q}-I_{h} \mathbf{q}\right)+\left(\mathbf{e}_{\mathbf{q}}, \boldsymbol{\sigma}-\Pi_{k}^{R T} \boldsymbol{\sigma}\right)+\left(\mathbf{e}_{\mathbf{q}}, \boldsymbol{\sigma}_{h}^{*}-\boldsymbol{\sigma}_{h}\right) \\
& -\left(\mathbf{e}_{\boldsymbol{\sigma}}, \mathbf{q}-I_{h} \mathbf{q}\right)+\left(\mathbf{b} e_{u}, \mathbf{q}-I_{h} \mathbf{q}\right)-\left(\mathbf{b} e_{u}, \mathbf{e}_{\mathbf{q}}\right) .
\end{aligned}
$$

Now, using the Cauchy-Schwarz inequality yields

$$
\begin{aligned}
\left\|a^{\frac{1}{2}} \mathbf{e}_{\mathbf{q}}\right\|^{2} \leq & C\left(\left\|\mathbf{q}-I_{h} \mathbf{q}\right\|+\left\|\boldsymbol{\sigma}-\Pi_{k}^{R T} \boldsymbol{\sigma}\right\|+\left\|\boldsymbol{\sigma}_{h}^{*}-\boldsymbol{\sigma}_{h}\right\|+\left\|e_{u}\right\|\right)\left\|a^{\frac{1}{2}} \mathbf{e}_{\mathbf{q}}\right\| \\
& +\left(\left\|\mathbf{e}_{\boldsymbol{\sigma}}\right\|+M\left\|e_{u}\right\|\right)\left\|\mathbf{q}-I_{h} \mathbf{q}\right\| .
\end{aligned}
$$

We apply triangle inequality to arrive at

$$
\left\|\boldsymbol{\sigma}_{h}^{*}-\boldsymbol{\sigma}_{h}\right\| \leq\left\|\boldsymbol{\sigma}_{h}^{*}-\Pi_{k}^{R T} \boldsymbol{\sigma}_{h}\right\|+\left\|\Pi_{k}^{R T} \boldsymbol{\sigma}_{h}-\boldsymbol{\sigma}_{h}\right\| .
$$

Using Lemma 3.2, we obtain

$$
\left\|\boldsymbol{\sigma}_{h}^{*}-\boldsymbol{\sigma}_{h}\right\| \leq C \Theta_{k}
$$

where

$$
\Theta_{k}:=\left\|\boldsymbol{\sigma}_{h}-\Pi_{k}^{R T} \boldsymbol{\sigma}_{h}\right\|+\left|P_{\partial}^{k}\left(\hat{\boldsymbol{\sigma}}_{h}-\boldsymbol{\sigma}_{h}\right)\right|_{L^{2}(\Gamma ; h)} .
$$


Note that if $\boldsymbol{\sigma}_{h}$ is given by the RT method, then $\Theta_{k}=0$. Again observe that if $\mathbf{W}_{h}$ is included in the space of fluxes of the corresponding RT method, $\boldsymbol{\sigma}_{h}=\Pi_{k}^{R T} \boldsymbol{\sigma}_{h}$, and

$$
\Theta_{k}=\left|P_{\partial}^{k}\left(\hat{\boldsymbol{\sigma}}_{h}-\boldsymbol{\sigma}_{h}\right)\right|_{L^{2}(\Gamma ; h)} .
$$

An application of Lemma 3.3 along with a use of Young's inequality and $a \geq \alpha_{0} \geq 0$ yields

$$
\left\|\mathbf{e}_{\mathbf{q}}\right\|^{2} \leq C\left(\left\|\mathbf{q}-I_{h} \mathbf{q}\right\|^{2}+\left\|\boldsymbol{\sigma}-\Pi_{k}^{R T} \boldsymbol{\sigma}\right\|^{2}+\left\|\boldsymbol{\sigma}-\mathbf{L}_{h} \boldsymbol{\sigma}\right\|^{2}+\left\|e_{u}\right\|^{2}+\Theta_{k}^{2}\right) .
$$

This completes the rest of the proof.

3.5. Optimal convergence of $u_{h}$. In this subsection, we discuss the optimal estimate of $u-u_{h}$ in $L^{2}$-norm.

Lemma 3.5. For any method in the general form (3.3a)-3.3c), there exists a positive constant $C$ independent of $h$ such that for small $h$,

$$
\begin{aligned}
\left\|u-u_{h}\right\| \leq & C h\left(\left\|\mathbf{q}-I_{h} \mathbf{q}\right\|+\left\|\boldsymbol{\sigma}-\Pi_{k}^{R T} \boldsymbol{\sigma}\right\|+\left\|\boldsymbol{\sigma}-\mathbf{L}_{h} \boldsymbol{\sigma}\right\|+\Theta_{k}\right) \\
& +C_{1}\left\{\sum_{i=1}^{N_{h}} h_{i}^{2}\left\|\nabla\left(u-P^{k} u\right)\right\|_{L^{2}\left(K_{i}\right)}^{2}\right\}^{\frac{1}{2}}+C_{2}\left\{h \max \left\{\bar{C}_{22}, \frac{1}{\underline{C}_{11}}\right\} T\right\}^{\frac{1}{2}},
\end{aligned}
$$

$$
T:=\int_{\Gamma} C_{11} \llbracket u_{h} \rrbracket^{2} d s+\int_{\Gamma_{I}} C_{22} \llbracket \boldsymbol{\sigma}_{h} \rrbracket^{2} d s .
$$

Proof. We multiply (3.17c) by $e_{u},\left(3.17 \mathrm{~b}\right.$ ) by $\mathbf{e}_{\mathbf{q}}$ and (3.17a) by $\mathbf{e}_{\boldsymbol{\sigma}}$ to obtain

$$
\left(e_{u}, \lambda\right)=\left(e_{u}, \nabla \cdot \boldsymbol{\chi}\right)+\left(\mathbf{b} e_{u}, \mathbf{p}\right)+\left(\mathbf{e}_{\mathbf{q}}, \boldsymbol{\chi}\right)+\left(a \mathbf{e}_{\mathbf{q}}, \mathbf{p}\right)-\left(\mathbf{e}_{\boldsymbol{\sigma}}, \mathbf{p}\right)+\left(\mathbf{e}_{\boldsymbol{\sigma}}, \nabla \phi\right) .
$$

Then, using (3.26a $-(3.26 \mathrm{~b})$, we arrive at

$$
\begin{aligned}
\left(e_{u}, \lambda\right)= & \left(e_{u}, \nabla \cdot\left(\boldsymbol{\chi}-P^{k} \boldsymbol{\chi}\right)\right)+\left(\mathbf{b} e_{u}, \mathbf{p}-P^{k} \mathbf{p}\right)+\left(\mathbf{e}_{\mathbf{q}}, \boldsymbol{\chi}-P^{k} \boldsymbol{\chi}\right) \\
& +\left\langle u-\hat{u}_{h}, P^{k} \boldsymbol{\chi} \cdot \boldsymbol{\nu}\right\rangle+\left(a \mathbf{e}_{\mathbf{q}}, \mathbf{p}-P^{k} \mathbf{p}\right)-\left(\mathbf{e}_{\boldsymbol{\sigma}}, \mathbf{p}-P^{k} \mathbf{p}\right)+\left(\mathbf{e}_{\boldsymbol{\sigma}}, \nabla \phi\right) .
\end{aligned}
$$

Since $\llbracket \phi \rrbracket=0, \llbracket \chi \rrbracket=0$ on $e_{k} \in \Gamma_{I}$ and $\phi=0$ on $\partial \Omega$, we write (3.40) as

$$
\begin{aligned}
\left(e_{u}, \lambda\right)= & \left(e_{u}, \nabla \cdot\left(\boldsymbol{\chi}-P^{k} \chi\right)\right)+\left(\mathbf{b} e_{u}, \mathbf{p}-P^{k} \mathbf{p}\right)+\left(\mathbf{e}_{\mathbf{q}}, \boldsymbol{\chi}-P^{k} \boldsymbol{\chi}\right) \\
& -\left\langle u-\hat{u}_{h},\left(\boldsymbol{\chi}-P^{k} \boldsymbol{\chi}\right) \cdot \boldsymbol{\nu}\right\rangle+\left(a \mathbf{e}_{\mathbf{q}}, \mathbf{p}-P^{k} \mathbf{p}\right)-\left(\mathbf{e}_{\boldsymbol{\sigma}}, \mathbf{p}-P^{k} \mathbf{p}\right) \\
& +\left(\mathbf{e}_{\boldsymbol{\sigma}}, \nabla \phi\right) \\
= & I_{1}+I_{2}+I_{3}+I_{4}+I_{5}+I_{6}+I_{7} .
\end{aligned}
$$

Now, an application of integration by parts with the properties of $P^{k}$ yields

$$
\begin{aligned}
I_{1}+I_{4}= & \left(e_{u}, \nabla \cdot\left(\chi-P^{k} \chi\right)\right)-\left\langle u-\hat{u}_{h},\left(\chi-P^{k} \chi\right) \cdot \boldsymbol{\nu}\right\rangle \\
= & -\left(\nabla e_{u}, \chi-P^{k} \chi\right)-\left\langle u_{h}-\hat{u}_{h},\left(\chi-P^{k} \chi\right) \cdot \boldsymbol{\nu}\right\rangle \\
= & -\left(\nabla\left(u-P^{k} u\right), \chi-P^{k} \chi\right)-\left\langle u_{h}-\hat{u}_{h},\left(\chi-P^{k} \chi\right) \cdot \boldsymbol{\nu}\right\rangle \\
\leq & C \sum_{i=1}^{N_{h}}\left\|\nabla\left(u-P^{k} u\right)\right\|_{L^{2}\left(K_{i}\right)}\left\|\left(\boldsymbol{\chi}-P^{k} \boldsymbol{\chi}\right) \cdot \boldsymbol{\nu}\right\|_{L^{2}\left(K_{i}\right)} \\
& +\sum_{i=1}^{N_{h}}\left\|u_{h}-\hat{u}_{h}\right\|_{L^{2}\left(\partial K_{i}\right)}\left\|\left(\boldsymbol{\chi}-P^{k} \boldsymbol{\chi}\right) \cdot \boldsymbol{\nu}\right\|_{L^{2}\left(\partial K_{i}\right)},
\end{aligned}
$$


and hence, using the Cauchy-Schwarz inequality and approximation properties of $P^{k}$, we obtain

$$
\begin{aligned}
I_{1}+I_{4} \leq & C \sum_{i=1}^{N_{h}} h_{i}\left\|\nabla\left(u-P^{k} u\right)\right\|_{L^{2}\left(K_{i}\right)}\|\chi\|_{\mathbf{H}^{1}\left(K_{i}\right)} \\
& +\sum_{i=1}^{N_{h}} h_{i}^{\frac{1}{2}}\left\|u_{h}-\hat{u}_{h}\right\|_{L^{2}\left(\partial K_{i}\right)}\|\chi\|_{\mathbf{H}^{1}\left(K_{i}\right)} \\
(3.42) \leq & C\left(\sum_{i=1}^{N_{h}} h_{i}^{2}\left\|\nabla\left(u-P^{k} u\right)\right\|_{L^{2}\left(K_{i}\right)}^{2}+\sum_{i=1}^{N_{h}} h_{i}\left\|u_{h}-\hat{u}_{h}\right\|_{L^{2}\left(\partial K_{i}\right)}^{2}\right)^{\frac{1}{2}}\|\chi\|_{\mathbf{H}^{1}(\Omega)} .
\end{aligned}
$$

To estimate $I_{2}, I_{3}, I_{5}$ and $I_{6}$ on the right-hand side of (3.41), an application of the Cauchy-Schwarz inequality with approximation properties of $P^{k}$ yields

$$
I_{2}+I_{3}+I_{5}+I_{6} \leq C h\left(\left\|\mathbf{e}_{\mathbf{q}}\right\|+\left\|\mathbf{e}_{\boldsymbol{\sigma}}\right\|+\left\|e_{u}\right\|\right)\left(\|\chi\|_{\mathbf{H}^{1}(\Omega)}+\|\mathbf{p}\|_{\mathbf{H}^{1}(\Omega)}\right) .
$$

We rewrite $I_{7}$ on the right-hand side of (3.41) as

$$
\begin{aligned}
I_{7} & =\left(\boldsymbol{\sigma}-\Pi_{k}^{R T} \boldsymbol{\sigma}, \nabla \phi\right)+\left(\Pi_{k}^{R T} \boldsymbol{\sigma}-\boldsymbol{\sigma}_{h}^{*}, \nabla \phi\right)+\left(\boldsymbol{\sigma}_{h}^{*}-\boldsymbol{\sigma}_{h}, \nabla \phi\right) \\
& =I_{7}^{1}+I_{7}^{2}+I_{7}^{3} .
\end{aligned}
$$

By the definition of the Raviart-Thomas projection $\Pi_{k}^{R T}$ given in (3.11), we rewrite $I_{7}^{1}$ as

$$
I_{7}^{1}=\left(\boldsymbol{\sigma}-\Pi_{k}^{R T} \boldsymbol{\sigma}, \nabla \phi-P^{k-1} \nabla \phi\right),
$$

and hence, we obtain, as $k \geq 1$,

$$
\left|I_{7}^{1}\right| \leq C h\left\|\boldsymbol{\sigma}-\Pi_{k}^{R T} \boldsymbol{\sigma}\right\|\|\phi\|_{H^{2}(\Omega)} .
$$

For $I_{7}^{2}$, we integrate by parts and use properties (i), (ii) and (iii) of Lemma 3.1 to obtain

$$
I_{7}^{2}=\left\langle\left(\Pi_{k}^{R T} \boldsymbol{\sigma}-\boldsymbol{\sigma}_{h}^{*}\right) \cdot \boldsymbol{\nu}, \phi\right\rangle_{\partial \Omega}=0 .
$$

Finally, for $I_{7}^{3}$, we note using the definition of $\boldsymbol{\sigma}_{h}^{*}$ given by (3.23), that

$$
\begin{aligned}
I_{7}^{3} & =\left(\boldsymbol{\sigma}_{h}^{*}-\boldsymbol{\sigma}_{h}, \nabla \phi-P^{k-1} \nabla \phi\right) \\
& \leq C h\left\|\boldsymbol{\sigma}_{h}^{*}-\boldsymbol{\sigma}_{h}\right\|\|\phi\|_{H^{2}(\Omega)} \\
& \leq C h\left(\left\|\boldsymbol{\sigma}_{h}^{*}-\Pi_{k}^{R T} \boldsymbol{\sigma}_{h}\right\|+\left\|\Pi_{k}^{R T} \boldsymbol{\sigma}_{h}-\boldsymbol{\sigma}_{h}\right\|\right)\|\phi\|_{H^{2}(\Omega)} .
\end{aligned}
$$

An application of Lemma 3.2 with the use of (3.36) now yields

$$
\left|I_{7}^{3}\right| \leq C h \Theta_{k}\|\phi\|_{H^{2}(\Omega)} .
$$

Combining (3.45)-(3.47) to (3.44), we obtain

$$
\left|I_{7}\right| \leq C h\left(\left\|\boldsymbol{\sigma}-\Pi_{k}^{R T} \boldsymbol{\sigma}\right\|+\Theta_{k}\right)\|\phi\|_{H^{2}(\Omega)} .
$$

Substitute estimates (3.42)-(3.43) and (3.48) in (3.41) and use regularity result (3.18) to arrive at

$$
\begin{aligned}
\left\|e_{u}\right\| \leq & C\left(h\left(\left\|e_{u}\right\|+\left\|\mathbf{e}_{\mathbf{q}}\right\|+\left\|\mathbf{e}_{\boldsymbol{\sigma}}\right\|+\left\|\boldsymbol{\sigma}-\Pi_{k}^{R T} \boldsymbol{\sigma}\right\|+\Theta_{k}\right)\right. \\
& \left.+\left\{\sum_{i=1}^{N_{h}} h_{i}^{2}\left\|\nabla\left(u-P^{k} u\right)\right\|_{L^{2}\left(K_{i}\right)}^{2}\right\}^{\frac{1}{2}}+\left\{\sum_{i=1}^{N_{h}} h_{i}\left\|\hat{u}_{h}-u_{h}\right\|_{L^{2}\left(\partial K_{i}\right)}^{2}\right\}^{\frac{1}{2}}\right) .
\end{aligned}
$$


Now we use Lemma 3.4 and Lemma 3.3 to find that

$$
\begin{aligned}
& \left\|e_{u}\right\| \leq \quad C h\left(\left\|\mathbf{q}-I_{h} \mathbf{q}\right\|+\left\|\boldsymbol{\sigma}-\Pi_{k}^{R T} \boldsymbol{\sigma}\right\|+\left\|\boldsymbol{\sigma}-\mathbf{L}_{h} \boldsymbol{\sigma}\right\|+\Theta_{k}+\left\|e_{u}\right\|\right) \\
& 3.50)+C\left\{\sum_{i=1}^{N_{h}} h_{i}^{2}\left\|\nabla\left(u-P^{k} u\right)\right\|_{L^{2}\left(K_{i}\right)}^{2}\right\}^{\frac{1}{2}}+C\left\{\sum_{i=1}^{N_{h}} h_{i}\left\|\hat{u}_{h}-u_{h}\right\|_{L^{2}\left(\partial K_{i}\right)}^{2}\right\}^{\frac{1}{2}} .
\end{aligned}
$$

Now, it remains to find the estimate of the last term on the right-hand side of (3.50).

To estimate the last term on right-hand side of (3.50), we use the definition of numerical flux (3.4a) to arrive at

$$
\begin{aligned}
\sum_{i=1}^{N_{h}} h_{i}\left\|\hat{u}_{h}-u_{h}\right\|_{L^{2}\left(\partial K_{i}\right)}^{2} & \leq C h \max \left\{\bar{C}_{22}, \frac{1}{\underline{C}_{11}}\right\}\left(\int_{\Gamma} C_{11} \llbracket u_{h} \rrbracket^{2} d s+\int_{\Gamma_{I}} C_{22} \llbracket \sigma_{h} \rrbracket^{2} d s\right) \\
& \leq C h \max \left\{\bar{C}_{22}, \frac{1}{\underline{C}_{11}}\right\} T,
\end{aligned}
$$

where

$$
T:=\int_{\Gamma} C_{11} \llbracket u_{h} \rrbracket^{2} d s+\int_{\Gamma_{I}} C_{22} \llbracket \boldsymbol{\sigma}_{h} \rrbracket^{2} d s .
$$

On substituting (3.51) in (3.50) and choosing $h$ sufficiently small, we obtain

$$
\begin{aligned}
\left\|e_{u}\right\| \leq & C h\left(\left\|\mathbf{q}-I_{h} \mathbf{q}\right\|+\left\|\boldsymbol{\sigma}-\Pi_{k}^{R T} \boldsymbol{\sigma}\right\|+\left\|\boldsymbol{\sigma}-\mathbf{L}_{h} \boldsymbol{\sigma}\right\|+\Theta_{k}\right) \\
& +C\left\{\sum_{i=1}^{N_{h}} h_{i}^{2}\left\|\nabla\left(u-P^{k} u\right)\right\|_{L^{2}\left(K_{i}\right)}^{2}\right\}^{\frac{1}{2}}+C\left\{h \max \left\{\bar{C}_{22}, \frac{1}{\underline{C}_{11}}\right\} T\right\}^{\frac{1}{2}},
\end{aligned}
$$

and this completes the rest of the proof.

To complete the estimate of $e_{u}$ and hence $\mathbf{e}_{\mathbf{q}}$ with $\mathbf{e}_{\boldsymbol{\sigma}}$, it remains to find the estimates of $T$ and $\Theta_{k}$.

3.6. Estimates of $T$ and $\Theta_{k}$. In this subsection, we find estimates for each of the terms $T$ and $\Theta_{k}$. Now, as in [12, we introduce two projections, $\boldsymbol{\Pi}_{k}$ and $\mathbb{P}_{k}$. In order to define these two projections, for each element $K \in \mathcal{T}_{h}$, we have to single out a particular face, which we denote by $e_{K}^{\tau}$. The precise way in which we pick this face is not relevant for the present study. For a given function $\mathbf{w} \in \mathbf{H}^{1}\left(\Omega_{h}\right)$ and an arbitrary element $K \in \mathcal{T}_{h}$, the restriction of $\boldsymbol{\Pi}_{k} \mathbf{w} \in \mathbf{W}_{h}$ to $K$ as the element of $\mathcal{P}^{k}(K)$ satisfying

$$
\begin{aligned}
\left(\boldsymbol{\Pi}_{k} \mathbf{w}-\mathbf{w}, \boldsymbol{\tau}\right)_{K} & =0, \quad \forall \boldsymbol{\tau} \in \mathcal{P}^{k-1}(K), \\
\left\langle\left(\boldsymbol{\Pi}_{k} \mathbf{w}-\mathbf{w}\right) \cdot \boldsymbol{\nu}, v\right\rangle_{e} & =0, \quad \forall v \in \mathcal{P}^{k}(e) \text { and all edges } e \neq e_{K}^{\tau} .
\end{aligned}
$$

For any $\xi \in H^{1}\left(\Omega_{h}\right)$, the function $\mathbb{P}_{k} \xi$ is the element of $V_{h}$ and defined as follows. For each $K \in \mathcal{T}_{h}, \mathbb{P}_{k} \xi_{\left.\right|_{K}}$ is the element of $\mathcal{P}^{k}(K)$ satisfying

$$
\begin{aligned}
\left(\mathbb{P}_{k} \xi-\xi, v\right)_{K} & =0, \quad \forall v \in \mathcal{P}^{k-1}(K), \\
\left\langle\mathbb{P}_{k} \xi-\xi, \mu\right\rangle_{e_{k}^{\tau}} & =0, \quad \forall \mu \in \mathcal{P}^{k}\left(e_{k}^{\tau}\right) .
\end{aligned}
$$

These projections are well defined and have optimal approximation properties; see 12 . 
Lemma 3.6. For any method of the form (3.3a)-(3.3c), the following equality holds:

$$
\begin{aligned}
& \left\|a^{\frac{1}{2}}\left(\boldsymbol{\Pi}_{k} \mathbf{q}-\mathbf{q}_{h}\right)\right\|^{2}+T_{1}=\left(\boldsymbol{\Pi}_{k} \mathbf{q}-\mathbf{q}, \boldsymbol{\Pi}_{k} \boldsymbol{\sigma}-\boldsymbol{\sigma}_{h}\right) \\
& \quad+\left(a\left(\boldsymbol{\Pi}_{k} \mathbf{q}-\mathbf{q}\right), \boldsymbol{\Pi}_{k} \mathbf{q}-\mathbf{q}_{h}\right) \\
& \quad-\left(\mathbf{b}\left(u-u_{h}\right), \boldsymbol{\Pi}_{k} \mathbf{q}-\mathbf{q}_{h}\right)-\left(\boldsymbol{\Pi}_{k} \boldsymbol{\sigma}-\boldsymbol{\sigma}, \boldsymbol{\Pi}_{k} \mathbf{q}-\mathbf{q}_{h}\right)-T_{2}-T_{3},
\end{aligned}
$$

where

$$
\begin{aligned}
& T_{1}:=\left\langle\left(\boldsymbol{\sigma}_{h}-\hat{\boldsymbol{\sigma}}_{h}\right) \cdot \boldsymbol{\nu}, u_{h}-\hat{u}_{h}\right\rangle, \\
& T_{2}:=\left\langle\left(\boldsymbol{\sigma}_{h}-\hat{\boldsymbol{\sigma}}_{h}\right) \cdot \boldsymbol{\nu}, u-\mathbb{P}_{k} u\right\rangle, \\
& T_{3}:=\left\langle\left(P_{\partial} \boldsymbol{\sigma}-\boldsymbol{\Pi}_{k} \boldsymbol{\sigma}\right) \cdot \boldsymbol{\nu}, u_{h}-\hat{u}_{h}\right\rangle .
\end{aligned}
$$

Proof. To prove (3.57), we now recall equations (3.26a)-(3.26c). Using the definition of the projections $\boldsymbol{\Pi}_{k}$ and $\mathbb{P}_{k}$, we rewrite the system (3.26a)-(3.26c) as

$$
\begin{aligned}
& \left(\boldsymbol{\Pi}_{k} \mathbf{q}-\mathbf{q}_{h}, \mathbf{w}_{h}\right)+\left(\mathbb{P}_{k} u-u_{h}, \nabla \cdot \mathbf{w}_{h}\right) \\
& \quad-\left\langle u-\hat{u}_{h}, \mathbf{w}_{h} \cdot \mathbf{n}\right\rangle=\left(\boldsymbol{\Pi}_{k} \mathbf{q}-\mathbf{q}, \mathbf{w}_{h}\right), \quad \forall \mathbf{w}_{h} \in \mathbf{W}_{h}, \\
& \quad\left(a\left(\mathbf{q}-\mathbf{q}_{h}\right), \boldsymbol{\tau}_{h}\right)+\left(\mathbf{b}\left(u-u_{h}\right), \boldsymbol{\tau}_{h}\right)-\left(\boldsymbol{\Pi}_{k} \boldsymbol{\sigma}-\boldsymbol{\sigma}_{h}, \boldsymbol{\tau}_{h}\right) \\
& \quad=-\left(\boldsymbol{\Pi}_{k} \boldsymbol{\sigma}-\boldsymbol{\sigma}, \boldsymbol{\tau}_{h}\right) \forall \boldsymbol{\tau}_{h} \in \mathbf{W}_{h}, \\
& \quad\left(\boldsymbol{\Pi}_{k} \boldsymbol{\sigma}-\boldsymbol{\sigma}_{h}, \nabla v_{h}\right)-\left\langle\left(\boldsymbol{\sigma}-\hat{\boldsymbol{\sigma}}_{h}\right) \cdot \boldsymbol{\nu}, v_{h}\right\rangle=0 \forall v_{h} \in V_{h} .
\end{aligned}
$$

Choose $\mathbf{w}_{h}=\boldsymbol{\Pi}_{k} \boldsymbol{\sigma}-\boldsymbol{\sigma}_{h}, \boldsymbol{\tau}_{h}=\boldsymbol{\Pi}_{k} \mathbf{q}-\mathbf{q}_{h}$ and $v_{h}=\mathbb{P}_{k} u-u_{h}$ in the above equations, and obtain

$$
\begin{aligned}
& \left(a\left(\boldsymbol{\Pi}_{k} \mathbf{q}-\mathbf{q}_{h}\right), \boldsymbol{\Pi}_{k} \mathbf{q}-\mathbf{q}_{h}\right)=\left(\boldsymbol{\Pi}_{k} \mathbf{q}-\mathbf{q}, \boldsymbol{\Pi}_{k} \boldsymbol{\sigma}-\boldsymbol{\sigma}_{h}\right) \\
& \quad+\left(a\left(\boldsymbol{\Pi}_{k} \mathbf{q}-\mathbf{q}\right), \boldsymbol{\Pi}_{k} \mathbf{q}-\mathbf{q}_{h}\right) \\
& \quad-\left(\mathbf{b}\left(u-u_{h}\right), \boldsymbol{\Pi}_{k} \mathbf{q}-\mathbf{q}_{h}\right)-\left(\boldsymbol{\Pi}_{k} \boldsymbol{\sigma}-\boldsymbol{\sigma}, \boldsymbol{\Pi}_{k} \mathbf{q}-\mathbf{q}_{h}\right) \\
& \quad+\left\langle u_{h}-\mathbb{P}_{k} u,\left(\boldsymbol{\Pi}_{k} \boldsymbol{\sigma}-\boldsymbol{\sigma}_{h}\right) \cdot \boldsymbol{\nu}\right\rangle+\left\langle u-\hat{u}_{h},\left(\boldsymbol{\Pi}_{k} \boldsymbol{\sigma}-\boldsymbol{\sigma}_{h}\right) \cdot \boldsymbol{\nu}\right\rangle \\
& \quad+\left\langle\left(\boldsymbol{\sigma}-\hat{\boldsymbol{\sigma}}_{h}\right) \cdot \boldsymbol{\nu}, \mathbb{P}_{k} u-u_{h}\right\rangle \\
& \quad=\left(\boldsymbol{\Pi}_{k} \mathbf{q}-\mathbf{q}, \boldsymbol{\Pi}_{k} \boldsymbol{\sigma}-\boldsymbol{\sigma}_{h}\right)+\left(a\left(\boldsymbol{\Pi}_{k} \mathbf{q}-\mathbf{q}\right), \boldsymbol{\Pi}_{k} \mathbf{q}-\mathbf{q}_{h}\right) \\
& \quad-\left(\mathbf{b}\left(u-u_{h}\right), \boldsymbol{\Pi}_{k} \mathbf{q}-\mathbf{q}_{h}-\left(\boldsymbol{\Pi}_{k} \boldsymbol{\sigma}-\boldsymbol{\sigma}, \boldsymbol{\Pi}_{k} \mathbf{q}-\mathbf{q}_{h}\right)\right)+I,
\end{aligned}
$$

where

$$
\begin{aligned}
I:= & \left\langle u-\mathbb{P}_{k} u,\left(\boldsymbol{\Pi}_{k} \boldsymbol{\sigma}-\boldsymbol{\sigma}_{h}\right) \cdot \boldsymbol{\nu}\right\rangle+\left\langle u_{h}-\hat{u}_{h},\left(\boldsymbol{\Pi}_{k} \boldsymbol{\sigma}-\boldsymbol{\sigma}_{h}\right) \cdot \boldsymbol{\nu}\right\rangle \\
& +\left\langle\left(\boldsymbol{\sigma}-\hat{\boldsymbol{\sigma}}_{h}\right) \cdot \boldsymbol{\nu}, \mathbb{P}_{k} u-u_{h}\right\rangle .
\end{aligned}
$$

By the definition of projection $P_{\partial}$, we note that

$$
\begin{aligned}
I= & \left\langle u-\mathbb{P}_{k} u,\left(\boldsymbol{\Pi}_{k} \boldsymbol{\sigma}-\boldsymbol{\sigma}_{h}\right) \cdot \boldsymbol{\nu}\right\rangle+\left\langle u_{h}-\hat{u}_{h},\left(\boldsymbol{\Pi} \boldsymbol{\sigma}-\boldsymbol{\sigma}_{h}\right) \cdot \boldsymbol{\nu}\right\rangle \\
& +\left\langle\left(P_{\partial} \boldsymbol{\sigma}-\hat{\boldsymbol{\sigma}}_{h}\right) \cdot \boldsymbol{\nu}, \mathbb{P}_{k} u-u_{h}\right\rangle .
\end{aligned}
$$

We rewrite (3.62) and then use definitions of the terms $T_{i}, i=1,2,3$, to obtain

$$
\begin{aligned}
I= & \left\langle u-\mathbb{P}_{k} u,\left(\boldsymbol{\Pi}_{k} \boldsymbol{\sigma}-\hat{\boldsymbol{\sigma}}_{h}\right) \cdot \boldsymbol{\nu}\right\rangle-T_{2}-T_{3} \\
& +\left\langle u_{h}-\hat{u}_{h},\left(P_{\partial} \boldsymbol{\sigma}-\hat{\boldsymbol{\sigma}}_{h}\right) \cdot \boldsymbol{\nu}\right\rangle-T_{1}+\left\langle\left(P_{\partial} \boldsymbol{\sigma}-\hat{\boldsymbol{\sigma}}_{h}\right) \cdot \boldsymbol{\nu}, \mathbb{P}_{k} u-u\right\rangle \\
& +\left\langle\left(P_{\partial} \boldsymbol{\sigma}-\hat{\boldsymbol{\sigma}}_{h}\right) \cdot \boldsymbol{\nu}, u-\hat{u}_{h}\right\rangle+\left\langle\left(P_{\partial} \boldsymbol{\sigma}-\hat{\boldsymbol{\sigma}}_{h}\right) \cdot \boldsymbol{\nu}, \hat{u}_{h}-u_{h}\right\rangle \\
= & \left\langle u-\mathbb{P}_{k} u,\left(\boldsymbol{\Pi}_{k} \boldsymbol{\sigma}-P_{\partial} \boldsymbol{\sigma}\right) \cdot \boldsymbol{\nu}\right\rangle-T_{2}-T_{3}-T_{1}+\left\langle\left(P_{\partial} \boldsymbol{\sigma}-\hat{\boldsymbol{\sigma}}_{h}\right) \cdot \boldsymbol{\nu}, u-\hat{u}_{h}\right\rangle .
\end{aligned}
$$


The result now follows from the fact that

$$
\left\langle u-\mathbb{P}_{k} u,\left(\boldsymbol{\Pi}_{k} \boldsymbol{\sigma}-P_{\partial} \boldsymbol{\sigma}\right) \cdot \boldsymbol{\nu}\right\rangle=0,
$$

and

$$
\left\langle\left(P_{\partial} \boldsymbol{\sigma}-\hat{\boldsymbol{\sigma}}_{h}\right) \cdot \boldsymbol{\nu}, u-\hat{u}_{h}\right\rangle=0,
$$

since the functions $\left(\boldsymbol{\Pi}_{k} \boldsymbol{\sigma}-P_{\partial} \boldsymbol{\sigma}\right) \cdot \boldsymbol{\nu}$ and $u-\hat{u}_{h}$ are single valued on all interior edges and since its product is zero on the boundary faces. This completes the proof.

Now using the definition of numerical fluxes $\hat{u}_{h}$ and $\hat{\boldsymbol{\sigma}}_{h}$, and the fact that it is single valued, we write $T_{1}$ as

$$
T_{1}=\int_{\Gamma} C_{11} \llbracket u_{h} \rrbracket^{2} d s+\int_{\Gamma_{I}} C_{22} \llbracket \boldsymbol{\sigma}_{h} \rrbracket^{2} d s=T,
$$

where $T_{1}$ is defined as in Lemma 3.6

Lemma 3.7. If $T$ and $\Theta_{k}$ are defined by (3.52) and (3.36), respectively, then there is a positive constant $C$ such that

$$
T \leq C \Upsilon h^{2 k+1}+\left\|u-u_{h}\right\|^{2},
$$

provided (3.6) with (3.7) is satisfied and further that

$$
\begin{aligned}
\Theta_{k}^{2} & \leq C h \max \left\{\frac{1}{\underline{C}_{22}}, \bar{C}_{11}\right\} T \\
& \leq C \Upsilon h^{2 k+2}+C h\left\|u-u_{h}\right\|^{2},
\end{aligned}
$$

where

$$
\Upsilon=|u|_{H^{k+1}\left(\Omega_{h}\right)}^{2}+|\mathbf{q}|_{\mathbf{H}^{k+1}\left(\Omega_{h}\right)}^{2}+|\boldsymbol{\sigma}|_{\mathbf{H}^{k+1}\left(\Omega_{h}\right)}^{2} .
$$

Proof. From Lemma 3.6, we replace $T_{1}$ in (3.57) by $T$ and apply the CauchySchwarz inequality and the Young's inequality with kick-back arguments to obtain

$$
\begin{aligned}
\frac{1}{2}\left\|a^{\frac{1}{2}}\left(\boldsymbol{\Pi}_{k} \mathbf{q}-\mathbf{q}_{h}\right)\right\|^{2}+T_{1} \leq & C\left(\left\|\boldsymbol{\Pi}_{k} \mathbf{q}-\mathbf{q}\right\|^{2}+\left\|\boldsymbol{\Pi}_{k} \boldsymbol{\sigma}-\boldsymbol{\sigma}\right\|^{2}+\left\|u-u_{h}\right\|^{2}\right) \\
& +\left|T_{2}\right|+\left|T_{3}\right| .
\end{aligned}
$$

To complete the proof of (3.66), it is sufficient to estimate $\left|T_{2}\right|$ and $\left|T_{3}\right|$. Now, from (3.59), the definition of $T_{2},(3.4)$, the definition of numerical flux $\hat{\boldsymbol{\sigma}}_{h}$, and application of the Cauchy-Schwarz inequality, we note that

$$
\begin{aligned}
\left|T_{2}\right| & \leq \sum_{i=1}^{N_{h}}\left\|\left(\boldsymbol{\sigma}-\hat{\boldsymbol{\sigma}}_{h}\right) \cdot \boldsymbol{\nu}\right\|_{L^{2}\left(\partial K_{i}\right)}\left\|u-\mathbb{P}_{k} u\right\|_{L^{2}\left(\partial K_{i}\right)} \\
& \leq\left(\sum_{i=1}^{N_{h}}\left\|\left(\boldsymbol{\sigma}-\hat{\boldsymbol{\sigma}}_{h}\right) \cdot \boldsymbol{\nu}\right\|_{L^{2}\left(\partial K_{i}\right)}^{2}\right)^{\frac{1}{2}}\left(\sum_{i=1}^{N_{h}}\left\|u-\mathbb{P}_{k} u\right\|_{L^{2}\left(\partial K_{i}\right)}^{2}\right)^{\frac{1}{2}} \\
& \leq C\left(\bar{C}_{11} \int_{\Gamma} C_{11} \llbracket u_{h} \rrbracket^{2} d s+\frac{1}{\underline{C}_{22}} \int_{\Gamma_{I}} C_{22} \llbracket \boldsymbol{\sigma}_{h} \rrbracket^{2} d s\right)^{\frac{1}{2}}\left(\sum_{i=1}^{N_{h}}\left\|u-\mathbb{P}_{k} u\right\|_{L^{2}\left(\partial K_{i}\right)}^{2}\right)^{\frac{1}{2}} \\
& \leq \frac{1}{4} T+C\left(\bar{C}_{11}+\frac{1}{\underline{C}_{22}}\right) \sum_{i=1}^{N_{h}}\left\|u-\mathbb{P}_{k} u\right\|_{L^{2}\left(\partial K_{i}\right)}^{2} .
\end{aligned}
$$


Similarly,

$$
\begin{aligned}
\left|T_{3}\right| \leq & \sum_{i=1}^{N_{h}}\left\|u-\hat{u}_{h}\right\|_{L^{2}\left(\partial K_{i}\right)}\left\|\left(P_{\partial} \boldsymbol{\sigma}-\Pi_{k} \boldsymbol{\sigma}\right) \cdot \boldsymbol{\nu}\right\|_{L^{2}\left(\partial K_{i}\right)} \\
\leq & \left(\sum_{i=1}^{N_{h}}\left\|u-\hat{u}_{h}\right\|_{L^{2}\left(\partial K_{i}\right)}^{2}\right)^{\frac{1}{2}}\left(\sum_{i=1}^{N_{h}}\left\|\left(P_{\partial} \boldsymbol{\sigma}-\Pi_{k} \boldsymbol{\sigma}\right) \cdot \boldsymbol{\nu}\right\|_{L^{2}\left(\partial K_{i}\right)}^{2}\right)^{\frac{1}{2}} \\
\leq & C\left(\frac{1}{\underline{C}_{11}} \int_{\Gamma} C_{11} \llbracket u_{h} \rrbracket^{2} d s+\bar{C}_{22} \int_{\Gamma_{I}} C_{22} \llbracket \boldsymbol{\sigma}_{h} \rrbracket^{2} d s\right)^{\frac{1}{2}} \\
& \times\left(\sum_{i=1}^{N_{h}}\left\|\left(P_{\partial} \boldsymbol{\sigma}-\Pi_{k} \boldsymbol{\sigma}\right) \cdot \boldsymbol{\nu}\right\|_{L^{2}\left(\partial K_{i}\right)}^{2}\right)^{\frac{1}{2}} .
\end{aligned}
$$

An application of Young's inequality yields

$$
\left|T_{3}\right| \leq \frac{1}{4} T+C\left(\frac{1}{\underline{C}_{11}}+\bar{C}_{22}\right) \sum_{i=1}^{N_{h}}\left\|\left(P_{\partial} \boldsymbol{\sigma}-\Pi_{k} \boldsymbol{\sigma}\right) \cdot \boldsymbol{\nu}\right\|_{L^{2}\left(\partial K_{i}\right)}^{2} .
$$

On substituting estimates (3.67)- (3.69) in (3.66) and using approximation properties of the projection $\Pi_{k}, \mathbb{P}_{k}$ and $P_{\partial}$, we arrive at

$$
T \leq C \Upsilon \max \left\{\bar{C}_{11}+\frac{1}{\underline{C}_{22}}, \bar{C}_{22}+\frac{1}{\underline{C}_{11}}\right\} h^{2 k+1}+\left\|u-u_{h}\right\|^{2},
$$

where $\Upsilon=|u|_{H^{k+1}\left(\Omega_{h}\right)}^{2}+|\mathbf{q}|_{\mathbf{H}^{k+1}\left(\Omega_{h}\right)}^{2}+|\boldsymbol{\sigma}|_{\mathbf{H}^{k+1}\left(\Omega_{h}\right)}^{2}$. This completes the estimate (3.64).

In order to obtain the second estimate (3.65), we again use the form of numerical fluxes (3.4a)-(3.4b) and (3.5a)-(3.5b) and obtain by simple algebraic manipulation that

$$
\Theta_{k}^{2} \leq C h \max \left\{\frac{1}{\underline{C}_{22}}, \bar{C}_{11}\right\} T .
$$

On substituting the estimate for $T$, from (3.64), we arrive at

$$
\begin{aligned}
\Theta_{k}^{2} \leq & C \Upsilon \max \left\{\frac{1}{\underline{C}_{22}}, \bar{C}_{11}\right\} \max \left\{\bar{C}_{11}+\frac{1}{\underline{C}_{22}}, \bar{C}_{22}+\frac{1}{\underline{C}_{11}}\right\} h^{2 k+2} \\
& + \text { Ch } \max \left\{\frac{1}{\underline{C}_{22}}, \bar{C}_{11}\right\}\left\|u-u_{h}\right\|^{2} .
\end{aligned}
$$

This completes the rest of the proof.

Remark 3.1. For the LDG-H methods which are characterized by (3.8a $-(3.8 \mathrm{~b})$, we note that

$$
\hat{\boldsymbol{\sigma}}_{h}=\boldsymbol{\sigma}_{h}+\tau\left(u_{h}-\hat{u}_{h}\right) \boldsymbol{\nu}
$$

where $\tau$ is a nonnegative, piecewise constant, double valued function on the interior faces of triangulation and single valued on boundary faces. Here, $\hat{u}_{h}$ is an unknown on the interior faces, but is equal to zero on $\partial \Omega$. We note that the results (3.64)(3.65) of Lemma 3.7 are valid under the following conditions on $\tau$ :

$$
\begin{aligned}
& \text { (i) } \bar{\tau} \leq C_{1} \text {, } \\
& \text { (ii) } \frac{\bar{\tau}}{\tau} \leq C_{2} \text {, }
\end{aligned}
$$


for some constants $C_{1}$ and $C_{2}$, where $\bar{\tau}:=\max _{K \in \mathcal{T}_{h}} \bar{\tau}_{K}$ and $\underline{\tau}:=\left.\min _{K \in \mathcal{T}_{h}} \underline{\tau}\right|_{e_{K}^{\tau}}$. Here, for each element $K \in \mathcal{T}_{h}$, we set $e_{K}^{\tau}$ as an edge in which $\left.\tau\right|_{\partial K}$ is maximum. Let $\bar{\tau}_{K}$ be the maximum value of $\left.\tau\right|_{\partial K \backslash e_{K}^{\tau}}$.

Below, we state and prove our main result for the linear nonselfadjoint elliptic problem.

Theorem 3.2. Let $\left(u_{h}, \mathbf{q}_{h}, \boldsymbol{\sigma}_{h}\right) \in V_{h} \times \mathbf{W}_{h} \times \mathbf{W}_{h}$ satisfy (3.3a)-(3.3c). Then there exist a positive constant independent of $h$ such that

$$
\left\|u-u_{h}\right\|+\left\|\mathbf{q}-\mathbf{q}_{h}\right\|+\left\|\boldsymbol{\sigma}-\boldsymbol{\sigma}_{h}\right\| \leq C h^{k+1} .
$$

Proof. On substituting (3.64) and (3.65) in Lemma 3.5] and choosing $h$ sufficiently small, we easily obtain the estimate for $\left\|u-u_{h}\right\|$. Then, we substitute the estimate of $\left\|u-u_{h}\right\|$ in Lemma 3.3 and Lemma 3.4 and use properties of projection $\Pi_{k}^{R T}$ to obtain the estimates of $\left\|\mathbf{q}-\mathbf{q}_{h}\right\|$ and $\left\|\boldsymbol{\sigma}-\boldsymbol{\sigma}_{h}\right\|$, respectively. This completes the rest of the proof.

Remark 3.2. As in [15], it is possible to postprocess the discrete solution $u_{h}$ and obtain a superconvergence result. But we shall not pursue it here as the proof technique exactly goes parallel to [15].

\section{Quasilinear Elliptic Problems}

In this section, we consider the following nonlinear elliptic boundary value problem:

$$
\left\{\begin{aligned}
\nabla \cdot(a(u) \nabla u) & =f(x), & & \text { in } \Omega \\
u & =0, & & \text { on } \partial \Omega .
\end{aligned}\right.
$$

Introduce new variables $\mathbf{q}=\nabla u$ and $\boldsymbol{\sigma}=a(u) \mathbf{q}$ and rewrite (4.1) as

$$
\left\{\begin{aligned}
\mathbf{q} & =\nabla u, & & \text { in } \Omega, \\
\boldsymbol{\sigma} & =a(u) \mathbf{q}, & & \text { in } \Omega, \\
-\nabla \cdot \boldsymbol{\sigma} & =f, & & \text { in } \Omega, \\
u & =0, & & \text { on } \partial \Omega .
\end{aligned}\right.
$$

In our subsequent analysis, we use the following Taylor series expansion: for $s$ and $\tau \in \mathbb{R}$

$$
a(s)=a(\tau)+\tilde{a}_{\tau}(s)(s-\tau)
$$

where $\tilde{a}_{\tau}(s)=\int_{0}^{1} a_{\tau}(\tau+t(s-\tau)) d t$, and

$$
a(s)=a(\tau)+a_{\tau}(\tau)(s-\tau)+\tilde{a}_{\tau \tau}(s)(s-\tau)^{2},
$$

where $\tilde{a}_{\tau \tau}(s)=\int_{0}^{1}(1-t) a_{\tau \tau}(\tau+t(s-\tau)) d t$.

4.1. DG formulation. We first introduce the DG formulation for our nonlinear elliptic problem (4.2): seek $\left(u_{h}, \mathbf{q}_{h}, \boldsymbol{\sigma}_{h}\right) \in V_{h} \times \mathbf{W}_{h} \times \mathbf{W}_{h}$ satisfying
(4.5a) $\left(\mathbf{q}_{h}, \mathbf{w}_{h}\right)+\left(u_{h}, \nabla \cdot \mathbf{w}_{h}\right)-\left\langle\hat{u}_{h}, \mathbf{w}_{h} \cdot \boldsymbol{\nu}\right\rangle=0$,
$\forall \mathbf{w}_{h} \in \mathbf{W}_{h}$,
$\left(a\left(u_{h}\right) \mathbf{q}_{h}, \boldsymbol{\tau}_{h}\right)-\left(\boldsymbol{\sigma}_{h}, \boldsymbol{\tau}_{h}\right)=0$,
$\forall \boldsymbol{\tau}_{h} \in \mathbf{W}_{h}$,

$$
\left(\boldsymbol{\sigma}_{h}, \nabla v_{h}\right)-\left\langle\hat{\boldsymbol{\sigma}}_{h} \cdot \boldsymbol{\nu}, v_{h}\right\rangle=\left(f, v_{h}\right), \quad \forall v_{h} \in V_{h},
$$


where the numerical fluxes $\hat{u}_{h}$ and $\hat{\boldsymbol{\sigma}}_{h}$ are defined by (3.4a)-3.4b) on interior edges and by (3.5a)- $(3.5 \mathrm{~b})$ on boundary edges.

Now, we introduce some bilinear functionals to be used in our subsequent analysis. For $(\phi, \mathbf{p}),(v, \mathbf{w}) \in V \times \mathbf{W}$, define the bilinear functional:

$A: \mathbf{W} \times \mathbf{W} \rightarrow \mathbb{R}$ as

$$
A(\mathbf{p}, \mathbf{w})=\int_{\Omega} \mathbf{p} \cdot \mathbf{w} d x
$$

$A_{1}: \mathbf{W} \times V \rightarrow \mathbb{R}$ as

$$
\begin{aligned}
A_{1}(\mathbf{p} ; v) & \left.=\sum_{i=1}^{N_{h}} \int_{K_{i}} \mathbf{p} \cdot \nabla v d x-\int_{\Gamma}(\{\mathbf{p}\})-\mathbf{C}_{12} \llbracket \mathbf{p} \rrbracket\right) \llbracket v \rrbracket d s, \\
& =-\sum_{i=1}^{N_{h}} \int_{K_{i}} v \nabla \cdot \mathbf{p}+\int_{\Gamma_{I}}\left(\{v v\}+\mathbf{C}_{12} \cdot \llbracket v \rrbracket\right) \llbracket \mathbf{p} \rrbracket d s,
\end{aligned}
$$

$J_{1}: \mathbf{W} \times \mathbf{W} \rightarrow \mathbb{R}$ as

$$
J_{1}(\mathbf{p}, \mathbf{w})=\int_{\Gamma_{I}} C_{22} \llbracket \mathbf{p} \rrbracket \llbracket \mathbf{w} \rrbracket d s
$$

$A_{2}: \mathbf{W} \times \mathbf{W} \rightarrow \mathbb{R}$ as

$$
A_{2}(u ; \mathbf{p}, \mathbf{w})=\int_{\Omega} a(u) \mathbf{p} \cdot \mathbf{w} d x
$$

and $J: V \times V \rightarrow \mathbb{R}$ as

$$
J(\phi, v)=\int_{\Gamma} C_{11} \llbracket \phi \rrbracket \llbracket v \rrbracket d s .
$$

We also define the linear functional $L: V \rightarrow \mathbb{R}$ as

$$
L(v)=\int_{\Omega} f v d x .
$$

Using the above definitions, we rewrite the problem (4.5a)-4.5c) in compact form as: find $\left(u_{h}, \mathbf{q}_{h}, \boldsymbol{\sigma}_{h}\right) \in V_{h} \times \mathbf{W}_{h} \times \mathbf{W}_{h}$ such that

$$
\begin{aligned}
& \text { (4.6a) } A\left(\mathbf{q}_{h}, \mathbf{w}_{h}\right)-A_{1}\left(\mathbf{w}_{h}, u_{h}\right)+J_{1}\left(\boldsymbol{\sigma}_{h}, \mathbf{w}_{h}\right)=0, \quad \forall \mathbf{w}_{h} \in \mathbf{W}_{h}, \\
& A_{2}\left(u_{h} ; \mathbf{q}_{h}, \boldsymbol{\tau}_{h}\right)-A\left(\boldsymbol{\sigma}_{h}, \boldsymbol{\tau}_{h}\right)=0, \quad \forall \boldsymbol{\tau}_{h} \in \mathbf{W}_{h}, \\
& A_{1}\left(\boldsymbol{\sigma}_{h}, v_{h}\right)+J\left(u_{h}, v_{h}\right)=L\left(v_{h}\right), \quad \forall v_{h} \in V_{h} .
\end{aligned}
$$

Since $V_{h}$ and $\mathbf{W}_{h}$ are finite dimensional, the system (4.6a)-(4.6c) gives rise to a system of nonlinear equations. We shall discuss its solvability in subsection 4.3.

Since the numerical fluxes $\hat{u}_{h}$ and $\hat{\boldsymbol{\sigma}}_{h}$ are consistent, we obtain the following error equations:

(4.7a) $A\left(\mathbf{q}-\mathbf{q}_{h}, \mathbf{w}_{h}\right)-A_{1}\left(\mathbf{w}_{h}, u-u_{h}\right)+J_{1}\left(\boldsymbol{\sigma}-\boldsymbol{\sigma}_{h}, \mathbf{w}_{h}\right)=0, \quad \forall \mathbf{w}_{h} \in \mathbf{W}_{h}$,

$$
\begin{aligned}
A_{2}\left(u ; \mathbf{q}, \boldsymbol{\tau}_{h}\right)-A_{2}\left(u_{h} ; \mathbf{q}_{h}, \boldsymbol{\tau}_{h}\right)-A\left(\boldsymbol{\sigma}-\boldsymbol{\sigma}_{h}, \boldsymbol{\tau}_{h}\right) & =0, \quad \forall \boldsymbol{\tau}_{h} \in \mathbf{W}_{h}, \\
A_{1}\left(\boldsymbol{\sigma}-\boldsymbol{\sigma}_{h}, v_{h}\right)+J\left(u-u_{h}, v_{h}\right) & =0, \quad \forall v_{h} \in V_{h} .
\end{aligned}
$$

Adding and subtracting $A_{2}\left(u ; \mathbf{q}_{h}, \boldsymbol{\tau}_{h}\right)$, we rewrite (4.7b) as

$$
A_{2}\left(u ; \mathbf{q}-\mathbf{q}_{h}, \boldsymbol{\tau}_{h}\right)-A\left(\boldsymbol{\sigma}-\boldsymbol{\sigma}_{h}, \boldsymbol{\tau}_{h}\right)=\int_{\Omega}\left(a\left(u_{h}\right)-a(u)\right) \mathbf{q}_{h} \cdot \boldsymbol{\tau}_{h} d x, \quad \forall \boldsymbol{\tau}_{h} \in \mathbf{W}_{h},
$$


and then using Taylor's expansions (4.3), we rewrite (4.8) as

$$
\begin{aligned}
& A_{2}\left(u ; \mathbf{q}-\mathbf{q}_{h}, \boldsymbol{\tau}_{h}\right)-A\left(\boldsymbol{\sigma}-\boldsymbol{\sigma}_{h}, \boldsymbol{\tau}_{h}\right)+\int_{\Omega} a_{u}(u)\left(u-u_{h}\right) \mathbf{q} \cdot \boldsymbol{\tau}_{h} d x \\
& =\int_{\Omega}\left(a\left(u_{h}\right)-a(u)\right)\left(\mathbf{q}_{h}-\mathbf{q}\right) \cdot \boldsymbol{\tau}_{h} d x+\int_{\Omega}\left(a\left(u_{h}\right)-a(u)-a_{u}(u)\left(u_{h}-u\right)\right) \mathbf{q} \cdot \boldsymbol{\tau}_{h} d x .
\end{aligned}
$$

For notational simplicity, we introduce for $\boldsymbol{\tau}, \mathbf{p}, \mathbf{q} \in \mathbf{W}$ and $\phi, v \in V$,

$$
\begin{aligned}
N(u, q ; \phi, \boldsymbol{\tau}) & =\int_{\Omega}\left(a_{u}(u) \mathbf{q}\right) \phi \cdot \boldsymbol{\tau} d x \\
N_{1}(v-u ; \mathbf{p}-\mathbf{q}, \boldsymbol{\tau}) & =\int_{\Omega}(a(v)-a(u))(\mathbf{p}-\mathbf{q}) \cdot \boldsymbol{\tau} d x \\
& =\int_{\Omega} \tilde{a}_{u}(v)(v-u)(\mathbf{p}-\mathbf{q}) \cdot \boldsymbol{\tau} d x
\end{aligned}
$$

and

$$
\begin{aligned}
N_{2}(v-u ; \mathbf{q}, \boldsymbol{\tau}) & =\int_{\Omega}\left(a(v)-a(u)-a_{u}(u)(v-u)\right) \mathbf{q} \cdot \boldsymbol{\tau} d x \\
& =\int_{\Omega} \tilde{a}_{u u}(v)(v-u)^{2} \mathbf{q} \cdot \boldsymbol{\tau} d x .
\end{aligned}
$$

Note that in the definition of $N_{1}$ and $N_{2}$, we have used Taylor's expansions (4.3)-(4.4). Hence, the system (4.7a)-(4.7c) takes the form

$$
\begin{array}{cl}
A\left(\mathbf{q}-\mathbf{q}_{h}, \mathbf{w}_{h}\right)-A_{1}\left(\mathbf{w}_{h}, u-u_{h}\right)+J_{1}\left(\boldsymbol{\sigma}-\boldsymbol{\sigma}_{h}, \mathbf{w}_{h}\right)=0, & \forall \mathbf{w}_{h} \in \mathbf{W}_{h}, \\
A_{2}\left(u ; \mathbf{q}-\mathbf{q}_{h}, \boldsymbol{\tau}_{h}\right)+N\left(u, \mathbf{q} ; u-u_{h}, \boldsymbol{\tau}_{h}\right)-A\left(\boldsymbol{\sigma}-\boldsymbol{\sigma}_{h}, \boldsymbol{\tau}_{h}\right) & \\
=N_{1}\left(u_{h}-u ; \mathbf{q}_{h}-\mathbf{q}, \boldsymbol{\tau}_{h}\right)+N_{2}\left(u_{h}-u ; \mathbf{q}, \boldsymbol{\tau}_{h}\right), & \forall \boldsymbol{\tau}_{h} \in \mathbf{W}_{h}, \\
A_{1}\left(\boldsymbol{\sigma}-\boldsymbol{\sigma}_{h}, v_{h}\right)+J\left(u-u_{h}, v_{h}\right)=0, & \forall v_{h} \in V_{h} .
\end{array}
$$

4.2. Intermediate projections. Given $(u, \mathbf{q}, \boldsymbol{\sigma})$, we define $\left(\tilde{u}_{h}, \tilde{\mathbf{q}}_{h}, \tilde{\boldsymbol{\sigma}}_{h}\right) \in V_{h} \times$ $\mathbf{W}_{h} \times \mathbf{W}_{h}$ satisfying

$$
\begin{array}{cc}
(4.10 \mathrm{a}) & A\left(\mathbf{q}-\tilde{\mathbf{q}}_{h}, \mathbf{w}_{h}\right)-A_{1}\left(\mathbf{w}_{h}, u-\tilde{u}_{h}\right)+J_{1}\left(\boldsymbol{\sigma}-\tilde{\boldsymbol{\sigma}}_{h}, \mathbf{w}_{h}\right)=0, \quad \forall \mathbf{w}_{h} \in \mathbf{W}_{h}, \\
& A_{2}\left(u ; \mathbf{q}-\tilde{\mathbf{q}}_{h}, \boldsymbol{\tau}_{h}\right)+N\left(u, \mathbf{q} ; u-\tilde{u}_{h}, \boldsymbol{\tau}_{h}\right) \\
(4.10 \mathrm{~b}) & -A\left(\boldsymbol{\sigma}-\tilde{\boldsymbol{\sigma}}_{h}, \boldsymbol{\tau}_{h}\right)=0, \quad \forall \boldsymbol{\tau}_{h} \in \mathbf{W}_{h}, \\
(4.10 \mathrm{c}) & A_{1}\left(\boldsymbol{\sigma}-\tilde{\boldsymbol{\sigma}}_{h}, v_{h}\right)+J\left(u-\tilde{u}_{h}, v_{h}\right)=0, \quad \forall v_{h} \in V_{h} .
\end{array}
$$

Note that, in view of (3.26) and identifying the coefficients $a$ and $\mathbf{b}$ with $a(u)$ and $a_{u}(u) \mathbf{q}$, respectively, we find that the formulation 4.10a -4.10c corresponds to the DG methods for the operator $M: H^{2}(\Omega) \cap H_{0}^{1}(\Omega) \rightarrow L^{2}(\Omega)$ given by

$$
M \phi=-\nabla \cdot\left(a(u) \nabla \phi+a_{u}(u) \mathbf{q} \phi\right) .
$$

Hence, on repeating arguments as in Section 3, we easily obtain the following results.

Theorem 4.1. For given $(u, \mathbf{q}, \boldsymbol{\sigma})$, if $\left(\tilde{u}_{h}, \tilde{\mathbf{q}}_{h}, \tilde{\boldsymbol{\sigma}}_{h}\right)$ is a solution of (4.10a)-(4.10c), then there is a constant $C$ independent of $h$ such that

$$
\left\|u-\tilde{u}_{h}\right\|+\left\|\mathbf{q}-\tilde{\mathbf{q}}_{h}\right\|+\left\|\boldsymbol{\sigma}-\tilde{\boldsymbol{\sigma}}_{h}\right\| \leq C h^{k+1}
$$

and

$$
J\left(u-\tilde{u}_{h}, u-\tilde{u}_{h}\right)+J_{1}\left(\boldsymbol{\sigma}-\tilde{\boldsymbol{\sigma}}_{h}, \boldsymbol{\sigma}-\tilde{\boldsymbol{\sigma}}_{h}\right) \leq C h^{2 k+1} .
$$


4.3. Existence and uniqueness of the discrete problem. In this subsection, we discuss solvability of the discrete system (4.6a)-(4.6c) of nonlinear equations. Essentially, existence of the discrete solution will be achieved through a fixed point argument and then uniqueness of solution will be proved completing the solvability of the discrete system.

For our future use, we define the following norm for $(v, \mathbf{w}) \in V \times \mathbf{W}$ as

$$
\|(v, \mathbf{w})\|^{2}=\left(\|v\|_{L^{4}(\Omega)}^{2}+\|\mathbf{w}\|^{2}+J(v, v)+J_{1}(\mathbf{w}, \mathbf{w})\right) .
$$

To formulate (4.6a)-(4.6c) in fixed point format, we define for a given $(z, \boldsymbol{\theta}) \in$ $V_{h} \times \mathbf{W}_{h}$, a map $S_{h}: V_{h} \times \mathbf{W}_{h} \rightarrow V_{h} \times \mathbf{W}_{h}$ by $S_{h}(z, \boldsymbol{\theta})=\left(y, \mathbf{q}_{l}\right)$ and $\boldsymbol{\sigma}_{l} \in \mathbf{W}_{h}$, where the triplet $\left(y, \mathbf{q}_{l}, \boldsymbol{\sigma}_{l}\right) \in V \times \mathbf{W}_{h} \times \mathbf{W}_{h}$ satisfies

$$
\begin{array}{cr}
A\left(\mathbf{q}-\mathbf{q}_{l}, \mathbf{w}_{h}\right)-A_{1}\left(\mathbf{w}_{h}, u-y\right)+J_{1}\left(\boldsymbol{\sigma}-\boldsymbol{\sigma}_{l}, \mathbf{w}_{h}\right)=0, & \forall \mathbf{w}_{h} \in \mathbf{W}_{h}, \\
A_{2}\left(u ; \mathbf{q}-\mathbf{q}_{l}, \boldsymbol{\tau}_{h}\right)+N\left(u, \mathbf{q} ; u-y, \boldsymbol{\tau}_{h}\right)-A\left(\boldsymbol{\sigma}-\boldsymbol{\sigma}_{l}, \boldsymbol{\tau}_{h}\right) & \\
=N_{1}\left(z-u ; \boldsymbol{\theta}-\mathbf{q}, \boldsymbol{\tau}_{h}\right)+N_{2}\left(z-u ; \mathbf{q}, \boldsymbol{\tau}_{h}\right), & \forall \boldsymbol{\tau}_{h} \in \mathbf{W}_{h}, \\
A_{1}\left(\boldsymbol{\sigma}-\boldsymbol{\sigma}_{l}, v_{h}\right)+J\left(u-y, v_{h}\right)=0, & \forall v_{h} \in V_{h} .
\end{array}
$$

For notational convenience, set

$$
\begin{array}{ll}
\eta_{u}:=u-\tilde{u}_{h}, \quad \boldsymbol{\eta}_{\mathbf{q}}:=\mathbf{q}-\tilde{\mathbf{q}}_{h}, \quad \boldsymbol{\eta}_{\boldsymbol{\sigma}}:=\boldsymbol{\sigma}-\tilde{\boldsymbol{\sigma}}_{h}, \\
\xi_{y}:=y-\tilde{u}_{h}, \quad \boldsymbol{\xi}_{\mathbf{q}}:=\mathbf{q}_{l}-\tilde{\mathbf{q}}_{h}, \quad \boldsymbol{\xi}_{\boldsymbol{\sigma}}:=\boldsymbol{\sigma}_{l}-\tilde{\boldsymbol{\sigma}}_{h} .
\end{array}
$$

For a given $(z, \boldsymbol{\theta}) \in V_{h} \times \mathbf{W}_{h}$, the problem (4.12a) 4.12c) leads to a system of linear equations in $y, \mathbf{q}_{l}$, and $\boldsymbol{\sigma}_{l}$. Hence, with an appropriate modification of arguments in Subsection 3.2 on existence and uniqueness of the discrete problem when DG methods are applied to a linear nonselfadjoint elliptic problem, we show that the system (4.12a)-(4.12c) has a unique solution. Hence, the map $S_{h}$ is well defined. For proving existence of the solution to the discrete problem (4.6a)-(4.6c), it is enough to prove that this map $S_{h}$ has a fixed point. First, we show that $S_{h}$ maps a ball $\mathcal{O}_{\delta}\left(\tilde{u}_{h}, \tilde{\mathbf{q}}_{h}\right)$ to itself, where

$$
\mathcal{O}_{\delta}\left(\tilde{u}_{h}, \tilde{\mathbf{q}}_{h}\right)=\left\{(z, \boldsymbol{\theta}) \in V_{h} \times \mathbf{W}_{h}: \|\left(z-\tilde{u}_{h}, \boldsymbol{\theta}-\tilde{\mathbf{q}}_{h} \| \leq \delta\right\},\right.
$$

and for $\varepsilon>0$,

$$
\delta=\frac{1}{h^{\varepsilon}}\left(\left\|\boldsymbol{\eta}_{\mathbf{q}}\right\|^{2}+\left\|\boldsymbol{\eta}_{\boldsymbol{\sigma}}\right\|^{2}+\left\|\eta_{u}\right\|_{L^{4}(\Omega)}^{2}+\int_{\Gamma} C_{11} \llbracket \eta_{u} \rrbracket^{2} d s+\int_{\Gamma_{I}} C_{22} \llbracket \boldsymbol{\eta}_{\boldsymbol{\sigma}} \rrbracket^{2} d s\right)^{\frac{1}{2}} .
$$

Using Lemma 2.1 and Lemma 2.3 with $r=4$, we note that

$$
\begin{aligned}
\left\|\eta_{u}\right\|_{L^{4}\left(K_{i}\right)} & \leq\left\|u-I_{h} u\right\|_{L^{4}\left(K_{i}\right)}+\left\|I_{h} u-\tilde{u}_{h}\right\|_{L^{4}\left(K_{i}\right)} \\
& \leq C h_{i}^{k+\frac{1}{2}}\|u\|_{H^{k+1}\left(K_{i}\right)}+C h_{i}^{-\frac{1}{2}}\left\|I_{h} u-\tilde{u}_{h}\right\|_{L^{2}\left(K_{i}\right)} \\
& \leq C h_{i}^{k+\frac{1}{2}}\|u\|_{H^{k+1}\left(K_{i}\right)}+C h_{i}^{-\frac{1}{2}}\left(\left\|u-I_{h} u\right\|_{L^{2}\left(K_{i}\right)}+\left\|u-\tilde{u}_{h}\right\|_{L^{2}\left(K_{i}\right)}\right) .
\end{aligned}
$$

By Lemma 2.1 and Theorem 4.1, we now obtain the following result in the form of a lemma.

Lemma 4.1. For each $K_{i} \in \mathcal{T}_{h}$, there exists a positive constant $C$ independent of $h$ such that

$$
\left\|\eta_{u}\right\|_{L^{4}\left(K_{i}\right)} \leq C h_{i}^{k+\frac{1}{2}}\|u\|_{H^{k+1}\left(K_{i}\right)} .
$$


An application of Theorem 4.1 with Lemma 4.1 yields

$$
\delta \leq C h^{k+\frac{1}{2}-\varepsilon} \text {. }
$$

In the following lemma, we derive bounds for nonlinear terms $N_{1}$ and $N_{2}$.

Lemma 4.2. Let $(z, \boldsymbol{\theta}) \in \mathcal{O}_{\delta}\left(\tilde{u}_{h}, \tilde{\mathbf{q}}_{h}\right)$. There exists a positive constant $C$ such that

$$
\left|N_{1}\left(z-u ; \boldsymbol{\theta}-\mathbf{q}, \boldsymbol{\tau}_{h}\right)+N_{2}\left(z-u ; \mathbf{q}, \boldsymbol{\tau}_{h}\right)\right| \leq C h^{k+\frac{1}{2}-\varepsilon} \delta\left\|\boldsymbol{\tau}_{h}\right\|_{L^{4}} .
$$

Furthermore,

$$
\left|N_{1}\left(z-u ; \boldsymbol{\theta}-\mathbf{q}, \boldsymbol{\tau}_{h}\right)+N_{2}\left(z-u ; \mathbf{q}, \boldsymbol{\tau}_{h}\right)\right| \leq C h^{k-\varepsilon} \delta\left\|\boldsymbol{\tau}_{h}\right\| .
$$

Proof. To estimate $N_{1}\left(z-u ; \boldsymbol{\theta}-\mathbf{q}, \boldsymbol{\tau}_{h}\right)$, we use the generalized Hölder's inequality, and then split $z-u:=\left(z-\tilde{u}_{h}\right)-\eta_{u}$, and $\boldsymbol{\theta}-\mathbf{q}:=\left(\boldsymbol{\theta}-\tilde{\mathbf{q}}_{h}\right)-\boldsymbol{\eta}_{\mathbf{q}}$ to arrive at

$$
\begin{aligned}
\left|N_{1}\left(z-u ; \boldsymbol{\theta}-\mathbf{q}, \boldsymbol{\tau}_{h}\right)\right| & =\left|\int_{\Omega} \tilde{a}_{u}(z-u)(\boldsymbol{\theta}-\mathbf{q}) \cdot \boldsymbol{\tau}_{h} d x\right| \\
& \leq C\|z-u\|_{L^{4}(\Omega)}\|\boldsymbol{\theta}-\mathbf{q}\|\left\|\boldsymbol{\tau}_{h}\right\|_{L^{4}(\Omega)} \\
(4.17) & \leq C\left(\left\|z-\tilde{u}_{h}\right\|_{L^{4}(\Omega)}+\left\|\eta_{u}\right\|_{L^{4}(\Omega)}\right)\left(\left\|\boldsymbol{\theta}-\tilde{\mathbf{q}}_{h}\right\|+\left\|\boldsymbol{\eta}_{\mathbf{q}}\right\|\right)\left\|\boldsymbol{\tau}_{h}\right\|_{L^{4}(\Omega)} .
\end{aligned}
$$

Now use the definition of $\delta$ and the bound $\left\|\eta_{u}\right\|_{L^{4}(\Omega)}+\left\|\boldsymbol{\eta}_{\mathbf{q}}\right\| \leq h^{\varepsilon} \delta$ in (4.17) to obtain

$$
\left|N_{1}\left(z-u ; \boldsymbol{\theta}-\mathbf{q}, \boldsymbol{\tau}_{h}\right)\right| \leq C \delta^{2}\left(1+h^{\varepsilon}\right)^{2}\left\|\boldsymbol{\tau}_{h}\right\|_{L^{4}(\Omega)} .
$$

For $N_{2}\left(z-u ; \mathbf{q}, \boldsymbol{\tau}_{h}\right)$, it is straightforward to check that

$$
\begin{aligned}
\left|N_{2}\left(z-u ; \mathbf{q}, \boldsymbol{\tau}_{h}\right)\right| & =\left|\int_{\Omega} \tilde{a}_{u u}(z-u)^{2} \mathbf{q} \cdot \boldsymbol{\tau}_{h} d x\right| \\
& \leq\left\|\tilde{a}_{u u} \mathbf{q}\right\|_{L^{4}(\Omega)}\|z-u\|_{L^{4}(\Omega)}^{2}\left\|\boldsymbol{\tau}_{h}\right\|_{L^{4}(\Omega)} \\
& \leq C \delta^{2}\left\|\boldsymbol{\tau}_{h}\right\|_{L^{4}(\Omega)} .
\end{aligned}
$$

We now combine (4.18) and (4.19). Then use the estimate (4.14) to bound one $\delta$ and arrive at

$$
\left|N_{1}\left(z-u ; \boldsymbol{\theta}-\mathbf{q}, \boldsymbol{\tau}_{h}\right)+N_{2}\left(z-u ; \mathbf{q}, \boldsymbol{\tau}_{h}\right)\right| \leq C h^{k+\frac{1}{2}-\varepsilon} \delta\left\|\boldsymbol{\tau}_{h}\right\|_{L^{4}(\Omega)} .
$$

This completes the estimate (4.15). To estimate (4.16), we use the inverse inequality (2.3) for $r=4$, and this completes the rest of the proof.

Since estimates of $\eta_{u}, \boldsymbol{\eta}_{\mathbf{q}}$, and $\boldsymbol{\eta}_{\boldsymbol{\sigma}}$ are known from Theorem 4.1, we need to derive in the following two lemmas, estimates $\xi_{y}, \boldsymbol{\xi}_{\mathbf{q}}$ and $\boldsymbol{\xi}_{\boldsymbol{\sigma}}$. Using mixed elliptic type projections (4.10a)-(4.10c and equations 4.12a)-(4.12c), we now write equations in $\xi_{y}, \boldsymbol{\xi}_{\mathbf{q}}$ and $\boldsymbol{\xi}_{\boldsymbol{\sigma}}$ as

(4.20a) $A\left(\boldsymbol{\xi}_{\mathbf{q}}, \mathbf{w}_{h}\right)-A_{1}\left(\mathbf{w}_{h}, \xi_{y}\right)+J_{1}\left(\boldsymbol{\xi}_{\boldsymbol{\sigma}}, \mathbf{w}_{h}\right)=0, \quad \forall \mathbf{w}_{h} \in \mathbf{W}_{h}$,

(4.20b) $\quad A_{2}\left(u ; \boldsymbol{\xi}_{\mathbf{q}}, \boldsymbol{\tau}_{h}\right)+N\left(u, \mathbf{q} ; \xi_{y}, \boldsymbol{\tau}_{h}\right)-A\left(\boldsymbol{\xi}_{\boldsymbol{\sigma}}, \boldsymbol{\tau}_{h}\right)$

$$
=-N_{1}\left(z-u ; \boldsymbol{\theta}-\mathbf{q}, \boldsymbol{\tau}_{h}\right)-N_{2}\left(z-u ; \mathbf{q}, \boldsymbol{\tau}_{h}\right), \quad \forall \boldsymbol{\tau}_{h} \in \mathbf{W}_{h},
$$

$$
A_{1}\left(\boldsymbol{\xi}_{\boldsymbol{\sigma}}, v_{h}\right)+J\left(\xi_{y}, v_{h}\right)=0, \quad \forall v_{h} \in V_{h} .
$$

In the following Lemma, we derive an estimate of $\boldsymbol{\xi}_{\mathbf{q}}$ and $\boldsymbol{\xi}_{\boldsymbol{\sigma}}$.

Lemma 4.3. There is a positive constant $C$ independent of $h$ such that

$$
\left\|\boldsymbol{\xi}_{\boldsymbol{\sigma}}\right\|+\left(\left\|\boldsymbol{\xi}_{\mathbf{q}}\right\|^{2}+J_{1}\left(\boldsymbol{\xi}_{\boldsymbol{\sigma}}, \boldsymbol{\xi}_{\boldsymbol{\sigma}}\right)+J\left(\xi_{y}, \xi_{y}\right)\right)^{\frac{1}{2}} \leq C\left(\left\|\xi_{y}\right\|+h^{k-\varepsilon} \delta\right) .
$$


Proof. Choose $\boldsymbol{\tau}_{h}=\boldsymbol{\xi}_{\boldsymbol{\sigma}}$ in (4.20b) to obtain

$$
\begin{aligned}
A\left(\boldsymbol{\xi}_{\boldsymbol{\sigma}}, \boldsymbol{\xi}_{\boldsymbol{\sigma}}\right)= & A_{2}\left(u ; \boldsymbol{\xi}_{\mathbf{q}}, \boldsymbol{\xi}_{\boldsymbol{\sigma}}\right)+N\left(u, q ; \xi_{y}, \boldsymbol{\xi}_{\boldsymbol{\sigma}}\right) \\
& N_{1}\left(z-u ; \boldsymbol{\theta}-\mathbf{q}, \boldsymbol{\xi}_{\boldsymbol{\sigma}}\right)+N_{2}\left(z-u ; \mathbf{q}, \boldsymbol{\xi}_{\boldsymbol{\sigma}}\right) .
\end{aligned}
$$

Now using the Cauchy-Schwarz inequality with (4.16) in (4.22) yields

$$
\left\|\boldsymbol{\xi}_{\boldsymbol{\sigma}}\right\| \leq C\left(\left\|\boldsymbol{\xi}_{\mathbf{q}}\right\|+\left\|\xi_{y}\right\|+h^{k-\varepsilon} \delta\right) .
$$

Choose $\boldsymbol{\tau}_{h}=\boldsymbol{\xi}_{\mathbf{q}}$ in (4.20b), $\mathbf{w}_{h}=\boldsymbol{\xi}_{\boldsymbol{\sigma}}$ in (4.20a) and $v_{h}=\xi_{u}$ in (4.20c). Then add the resulting equations to find that

$$
\begin{aligned}
A_{2}\left(u ; \boldsymbol{\xi}_{\mathbf{q}}, \boldsymbol{\xi}_{\mathbf{q}}\right) & +J_{1}\left(\boldsymbol{\xi}_{\boldsymbol{\sigma}}, \boldsymbol{\xi}_{\boldsymbol{\sigma}}\right)+J\left(\xi_{y}, \xi_{y}\right)=-N\left(u, \mathbf{q} ; \xi_{y}, \boldsymbol{\xi}_{\mathbf{q}}\right) \\
& -N_{1}\left(z-u ; \boldsymbol{\theta}-\mathbf{q}, \boldsymbol{\xi}_{\mathbf{q}}\right)-N_{2}\left(z-u ; \mathbf{q}, \boldsymbol{\xi}_{\mathbf{q}}\right) .
\end{aligned}
$$

Since $a \geq \alpha_{0}$, we obtain from (4.24) using the Cauchy-Schwarz inequality and boundedness property of $a_{u}$ :

$$
\begin{aligned}
\alpha_{0}\left\|\boldsymbol{\xi}_{\mathbf{q}}\right\|^{2}+J_{1}\left(\boldsymbol{\xi}_{\boldsymbol{\sigma}}, \boldsymbol{\xi}_{\boldsymbol{\sigma}}\right) & +J\left(\xi_{y}, \xi_{y}\right) \leq C\left\|\xi_{y}\right\|\left\|\boldsymbol{\xi}_{\mathbf{q}}\right\| \\
& +\left|N_{1}\left(z-u ; \boldsymbol{\theta}-\mathbf{q}, \boldsymbol{\xi}_{\mathbf{q}}\right)+N_{2}\left(z-u ; \mathbf{q}, \boldsymbol{\xi}_{\mathbf{q}}\right)\right| .
\end{aligned}
$$

Now, an application of the Cauchy-Schwarz inequality with the Young's inequality and (4.16) yields

$$
\left(\left\|\boldsymbol{\xi}_{\mathbf{q}}\right\|^{2}+J_{1}\left(\boldsymbol{\xi}_{\boldsymbol{\sigma}}, \boldsymbol{\xi}_{\boldsymbol{\sigma}}\right)+J\left(\xi_{y}, \xi_{y}\right)\right) \leq C\left(\left\|\xi_{y}\right\|^{2}+h^{2(k-\varepsilon)} \delta^{2}\right) .
$$

Now substitute (4.26) in (4.23) to complete the rest of the proof.

Lemma 4.4. Let $(z, \boldsymbol{\theta}) \in \mathcal{O}_{\delta}\left(\tilde{u}_{h}, \tilde{\mathbf{q}}_{h}\right)$ and let $\left(y, \mathbf{q}_{l}, \boldsymbol{\sigma}_{l}\right) \in V_{h} \times \mathbf{W}_{h} \times \mathbf{W}_{h}$ be the corresponding solution of (4.12a)-(4.12c). Then there exists a positive constant $C$ independent of $h$ such that

$$
\left\|\xi_{y}\right\| \leq C\left(\max \left\{h, \frac{1}{\underline{C}_{11}}, h^{2} \bar{C}_{11}, \bar{C}_{22}\right\}\right)^{\frac{1}{2}} h^{k+\frac{1}{2}-\varepsilon} \delta .
$$

Proof. We now apply the duality argument. Consider the following dual problem

$$
\begin{aligned}
& -\nabla \cdot(a(u) \nabla \phi)+a_{u}(u) \mathbf{q} \cdot \nabla \phi=\theta, \quad \text { in } \Omega, \\
& \phi=0, \quad \text { on } \partial \Omega \text {, }
\end{aligned}
$$

which satisfies the regularity result

$$
\|\phi\|_{H^{2}(\Omega)} \leq C\|\theta\| .
$$

With $\mathbf{p}=\nabla \phi,-\boldsymbol{\chi}=a(u) \mathbf{p}$, rewrite (4.28) as

$$
\begin{aligned}
\mathbf{p} & =\nabla \phi, & & \text { in } \Omega, \\
-\boldsymbol{\chi} & =a(u) \mathbf{p}, & & \text { in } \Omega, \\
\nabla \cdot \boldsymbol{\chi}+a_{u}(u) \mathbf{q} \cdot \mathbf{p} & =\theta, & & \text { in } \Omega .
\end{aligned}
$$

Multiply $\xi_{y}$ in (4.30c), $\boldsymbol{\xi}_{\mathbf{q}}$ in (4.30b) and $\boldsymbol{\xi}_{\boldsymbol{\sigma}}$ in (4.30a) and then integrate over $\Omega$ to arrive at

$$
\begin{aligned}
\left(\xi_{y}, \theta\right)= & \int_{\Omega} \xi_{y} \nabla \cdot \boldsymbol{\chi} d x+\int_{\Omega} a_{u}(u) \mathbf{q} \xi_{y} \cdot \mathbf{p} d x+\int_{\Omega} a(u) \mathbf{p} \cdot \boldsymbol{\xi}_{\mathbf{q}} d x+\int_{\Omega} \boldsymbol{\chi} \cdot \boldsymbol{\xi}_{\mathbf{q}} \\
& -\int_{\Omega} \mathbf{p} \cdot \boldsymbol{\xi}_{\boldsymbol{\sigma}} d x+\int_{\Omega} \nabla \phi \cdot \boldsymbol{\xi}_{\boldsymbol{\sigma}} d x .
\end{aligned}
$$


Since $\llbracket \phi \rrbracket=0, \llbracket \chi \rrbracket=0$ on $e_{k} \in \Gamma_{I}$ and $\phi=0$ on $\partial \Omega$, we easily obtain from (4.31)

$$
\begin{aligned}
\left(\xi_{y}, \theta\right)= & A\left(\boldsymbol{\xi}_{\mathbf{q}}, \boldsymbol{\chi}\right)-A_{1}\left(\boldsymbol{\chi}, \xi_{y}\right)+J_{1}\left(\boldsymbol{\xi}_{\boldsymbol{\sigma}}, \boldsymbol{\chi}\right)+A_{2}\left(u ; \boldsymbol{\xi}_{\mathbf{q}}, \mathbf{p}\right)+N\left(u, \mathbf{q} ; \xi_{y}, \mathbf{p}\right) \\
& -A\left(\boldsymbol{\xi}_{\boldsymbol{\sigma}}, \mathbf{p}\right)+A_{1}\left(\boldsymbol{\xi}_{\boldsymbol{\sigma}}, \phi\right)+J\left(\xi_{y}, \phi\right) .
\end{aligned}
$$

Using $\mathbf{L}^{2}$-projection and equations (4.20a)-(4.20c), we now find that

$$
\begin{aligned}
\left(\xi_{y}, \theta\right)= & A\left(\boldsymbol{\xi}_{\mathbf{q}}, \boldsymbol{\eta}_{\boldsymbol{\chi}}\right)-A_{1}\left(\boldsymbol{\eta}_{\boldsymbol{\chi}}, \xi_{y}\right)+J_{1}\left(\boldsymbol{\xi}_{\boldsymbol{\sigma}}, \boldsymbol{\eta}_{\boldsymbol{\chi}}\right)+A_{2}\left(u ; \boldsymbol{\xi}_{\mathbf{q}}, \boldsymbol{\eta}_{\mathbf{p}}\right) \\
& +N\left(u, \mathbf{q} ; \xi_{y}, \boldsymbol{\eta}_{\mathbf{p}}\right)-A\left(\boldsymbol{\xi}_{\boldsymbol{\sigma}}, \boldsymbol{\eta}_{\mathbf{p}}\right)+A_{1}\left(\boldsymbol{\xi}_{\boldsymbol{\sigma}}, \eta_{\phi}\right)+J\left(\xi_{y}, \eta_{\phi}\right) \\
& +N_{1}\left(z-u ; \boldsymbol{\theta}-\mathbf{q}, I_{h} \mathbf{p}\right)+N_{2}\left(z-u ; \mathbf{q}, I_{h} \mathbf{p}\right) \\
(4.32)= & I_{1}+I_{2}+I_{3}+I_{4}+I_{5}+I_{6}+I_{7}+I_{8}+I_{9}+I_{10},
\end{aligned}
$$

where $\eta_{\phi}=\phi-I_{h} \phi, \boldsymbol{\eta}_{\mathbf{p}}=\mathbf{p}-I_{h} \mathbf{p}$ and $\boldsymbol{\eta}_{\boldsymbol{\chi}}=\boldsymbol{\chi}-L_{h} \boldsymbol{\chi}$. Using approximation property of the $\mathbf{L}^{2}$-projection and $I_{h}$, we arrive at

$$
\left|I_{1}+I_{4}+I_{5}+I_{6}\right| \leq C h\left(\left\|\boldsymbol{\xi}_{\mathbf{q}}\right\|+\left\|\boldsymbol{\xi}_{\boldsymbol{\sigma}}\right\|+\left\|\xi_{y}\right\|\right)\|\mathbf{p}\|_{\mathbf{H}^{1}(\Omega)},
$$

as $\|\boldsymbol{\chi}\|_{\mathbf{H}^{1}(\Omega)} \leq C\|\mathbf{p}\|_{\mathbf{H}^{1}(\Omega)}$.

Since $L_{h} \boldsymbol{\chi}$ is the $\mathbf{L}^{2}$-projection of $\boldsymbol{\chi}$, the first term of $I_{2}$ vanishes. Then using the Cauchy-Schwarz inequality and Lemma 2.1, we bound $I_{2}$ as

$$
\begin{aligned}
I_{2} & =\sum_{i=1}^{N_{h}} \int_{K_{i}} \xi_{y} \nabla \cdot \boldsymbol{\eta}_{\boldsymbol{\chi}} d x-\int_{\Gamma_{I}}\left(\left\{\xi_{y} \rrbracket+\mathbf{C}_{12} \llbracket \xi_{y} \rrbracket\right) \llbracket \boldsymbol{\eta}_{\boldsymbol{\chi}} \rrbracket d s\right. \\
& \left.=\int_{\Gamma} \llbracket \xi_{y} \rrbracket\left\{\boldsymbol{\eta}_{\boldsymbol{\chi}}\right\}\right\} d s-\int_{\Gamma_{I}} \mathbf{C}_{12} \llbracket \xi_{y} \rrbracket \llbracket \boldsymbol{\eta}_{\boldsymbol{\chi}} \rrbracket d s \\
& \leq C\left(\frac{h}{\underline{C}_{11}} J\left(\xi_{y}, \xi_{y}\right)\right)^{\frac{1}{2}}\|\boldsymbol{\chi}\|_{\mathbf{H}^{1}(\Omega)},
\end{aligned}
$$

where $\underline{C}_{11}=\min \left\{C_{11}(x): x \in \Gamma\right\}$. Similarly, we estimate $I_{7}$ as

$$
\begin{aligned}
I_{7} & =\sum_{i=1}^{N_{h}} \int_{K_{i}} \boldsymbol{\xi}_{\boldsymbol{\sigma}} \cdot \nabla \eta_{\phi} d x-\int_{\Gamma}\left(\left\{\left\{\boldsymbol{\xi}_{\boldsymbol{\sigma}}\right\}\right\}-\mathbf{C}_{12} \llbracket \boldsymbol{\xi}_{\boldsymbol{\sigma}} \rrbracket\right) \llbracket \eta_{\phi} \rrbracket d s \\
& \leq C \sum_{i=1}^{N_{h}}\left\|\boldsymbol{\xi}_{\boldsymbol{\sigma}}\right\|_{L^{2}\left(K_{i}\right)}\left\|\nabla \eta_{\phi}\right\|_{L^{2}\left(K_{i}\right)}+\sum_{e_{k} \in \Gamma}\left\|\boldsymbol{\xi}_{\boldsymbol{\sigma}}\right\|_{L^{2}\left(e_{k}\right)}\left\|\eta_{\phi}\right\|_{L^{2}\left(e_{k}\right)} \\
& \leq C h\left\|\boldsymbol{\xi}_{\boldsymbol{\sigma}}\right\|\|\phi\|_{H^{2}(\Omega)}+\sum_{i=1}^{N_{h}} h_{i}^{-\frac{1}{2}}\left\|\boldsymbol{\xi}_{\boldsymbol{\sigma}}\right\|_{L^{2}\left(K_{i}\right)} h^{\frac{3}{2}}\|\phi\|_{H^{2}\left(K_{i}\right)} \\
& \leq C h\left\|\boldsymbol{\xi}_{\boldsymbol{\sigma}}\right\|\|\phi\|_{H^{2}(\Omega)} .
\end{aligned}
$$

For $I_{3}$, we note using the Cauchy-Schwarz inequality and the approximation property of $\mathbf{L}^{2}$-projection that

$$
\begin{aligned}
I_{3} & \leq\left|\int_{\Gamma_{I}} C_{22} \llbracket \boldsymbol{\xi}_{\boldsymbol{\sigma}} \rrbracket \llbracket \boldsymbol{\eta}_{\boldsymbol{\chi}} \rrbracket d s\right| \\
& \leq C \sum_{e_{k} \in \Gamma_{I}}\left(\int_{e_{k}} C_{22} \llbracket \boldsymbol{\xi}_{\boldsymbol{\sigma}} \rrbracket^{2} d s\right)^{\frac{1}{2}}\left(\int_{e_{k}} C_{22} \llbracket \boldsymbol{\eta}_{\boldsymbol{\chi}} \rrbracket^{2} d s\right)^{\frac{1}{2}} \\
& \leq C\left(h \bar{C}_{22} J_{1}\left(\boldsymbol{\xi}_{\boldsymbol{\sigma}}, \boldsymbol{\xi}_{\boldsymbol{\sigma}}\right)\right)^{1 / 2}\|\boldsymbol{\chi}\|_{\mathbf{H}^{1}(\Omega)} .
\end{aligned}
$$


For $I_{8}$, we apply the Cauchy-Schwarz inequality and property of $\mathbf{L}^{2}$-projection to obtain

$$
\begin{aligned}
I_{8} & \leq\left|\int_{\Gamma} C_{11} \llbracket \xi_{y} \rrbracket \llbracket \eta_{\phi} \rrbracket d s\right| \\
& \leq C \sum_{e_{k} \in \Gamma}\left(\int_{e_{k}} C_{11} \llbracket \xi_{y} \rrbracket^{2} d s\right)^{\frac{1}{2}}\left(\int_{e_{k}} C_{11} \llbracket \eta_{\phi} \rrbracket^{2} d s\right)^{\frac{1}{2}} \\
& \leq C\left(h^{3} \bar{C}_{11} J\left(\xi_{y}, \xi_{y}\right)\right)^{\frac{1}{2}}\|\phi\|_{H^{2}(\Omega)} .
\end{aligned}
$$

Finally, using Lemma 4.2 and $\left\|I_{h} \mathbf{p}\right\|_{\mathbf{L}^{4}(\Omega)} \leq C\|\mathbf{p}\|_{\mathbf{H}^{1}(\Omega)}$, we find that

$$
\begin{aligned}
I_{9}+I_{10} & \leq\left|N_{1}\left(z-u ; \boldsymbol{\theta}-\mathbf{q}, I_{h} \mathbf{p}\right)+N_{2}\left(z-u ; \mathbf{q}, I_{h} \mathbf{p}\right)\right| \\
& \leq C h^{k+\frac{1}{2}-\varepsilon} \delta\left\|I_{h} \mathbf{p}\right\|_{L^{4}(\Omega)} \\
& \leq C h^{k+\frac{1}{2}-\varepsilon} \delta\|\mathbf{p}\|_{\mathbf{H}^{1}(\Omega)} .
\end{aligned}
$$

We substitute estimates (4.33)-(4.37) in (4.32) and then use elliptic regularity (4.29) to obtain

$$
\begin{aligned}
\left\|\xi_{y}\right\| \leq & C_{1} h\left(\left\|\boldsymbol{\xi}_{\mathbf{q}}\right\|+\left\|\boldsymbol{\xi}_{\boldsymbol{\sigma}}\right\|+\left\|\xi_{y}\right\|\right)+C_{2}\left(\max \left\{\frac{h}{\underline{C}_{11}}, h^{3} \bar{C}_{11}\right\} J\left(\xi_{y}, \xi_{y}\right)\right)^{\frac{1}{2}} \\
& +C_{3}\left(h \bar{C}_{22} J_{1}\left(\boldsymbol{\xi}_{\boldsymbol{\sigma}}, \boldsymbol{\xi}_{\boldsymbol{\sigma}}\right)\right)^{\frac{1}{2}}+C h^{k+\frac{1}{2}-\varepsilon} \delta .
\end{aligned}
$$

Now, using Lemma 4.3 yields

$$
\left(1-C_{1} h\right)\left\|\xi_{y}\right\| \leq C\left(\left(\max \left\{h, \frac{1}{\underline{C}_{11}}, h^{2} \bar{C}_{11}, \bar{C}_{22}\right\}\right)^{\frac{1}{2}}\right) h^{k+\frac{1}{2}-\varepsilon} \delta .
$$

Choose $h$ small so that $\left(1-C_{1} h\right)>0$. Then we obtain from (4.39) the estimate (4.27) and this completes the proof of Lemma 4.4.

Below, we shall show that $S_{h}$ is a self-map defined on $\mathcal{O}_{\delta}\left(\tilde{u}_{h}, \tilde{\mathbf{q}}_{h}\right)$ for small $h$.

Theorem 4.2. For all $0<h<h_{0}$ with $h_{0}<1$ and $\varepsilon \in(0,1 / 2]$, there is a $\delta=\delta(h)>0$ such that $S_{h}$ maps $\mathcal{O}_{\delta}\left(\tilde{u}_{h}, \tilde{\mathbf{q}}_{h}\right)$ into itself.

Proof. Substitute (4.27) from Lemma 4.4 in (4.26) and then again for small $h$, we arrive at

$$
\left\|\boldsymbol{\xi}_{\boldsymbol{\sigma}}\right\|+\left(\left\|\boldsymbol{\xi}_{\mathbf{q}}\right\|^{2}+J_{1}\left(\boldsymbol{\xi}_{\boldsymbol{\sigma}}, \boldsymbol{\xi}_{\boldsymbol{\sigma}}\right)+J\left(\xi_{y}, \xi_{y}\right)\right)^{\frac{1}{2}} \leq C h^{k-\varepsilon} \delta .
$$

Using the inverse inequality we note that

$$
\left\|\xi_{y}\right\|_{L^{4}} \leq C h^{-\frac{1}{2}}\left\|\xi_{y}\right\| .
$$

Now using the estimate of $\xi_{y}$ as in (4.27), we obtain for $k \geq 1, \varepsilon \in(0,1 / 2]$ and for small $h$,

$$
\left\|\left(\xi_{y}, \boldsymbol{\xi}_{\mathbf{q}}\right)\right\| \leq C h^{k-\varepsilon} \delta \leq \delta .
$$

This completes the rest of the proof.

Below, we prove that the map $S_{h}$ is Lipschitz continuous in the ball $\mathcal{O}_{\delta}\left(\tilde{u}_{h}, \tilde{\mathbf{q}}_{h}\right)$. 
Theorem 4.3. Let $\left(z_{1}, \boldsymbol{\theta}_{1}\right),\left(z_{2}, \boldsymbol{\theta}_{2}\right) \in \mathcal{O}_{\delta}\left(\tilde{u}_{h}, \tilde{\mathbf{q}}_{h}\right)$ with $0<\delta<1$. Then for sufficiently small $h$ and $\varepsilon \in(0,1 / 2]$, there exists a positive constant $C$ independent of $h$ such that

$$
\left\|S_{h}\left(z_{1}, \boldsymbol{\theta}_{1}\right)-S_{h}\left(z_{2}, \boldsymbol{\theta}_{2}\right)\right\| \leq C h^{(k-\varepsilon)}\left\|\left(z_{1}, \boldsymbol{\theta}_{1}\right)-\left(z_{2}, \boldsymbol{\theta}_{2}\right)\right\| .
$$

Proof. Let $\left(y_{i}, \mathbf{q}_{i}\right)=S_{h}\left(z_{i}, \boldsymbol{\theta}_{i}\right)$ with $\mathbf{q}_{l i}=\mathbf{q}_{i}$ and $\boldsymbol{\sigma}_{l i}=\boldsymbol{\sigma}_{i}$, for $i=1$, 2. From Theorem 4.2 and the estimates (4.40), it follows that for $i=1,2$,

$$
\left(\left\|\mathbf{q}_{i}-\tilde{\mathbf{q}}_{h}\right\|+\left\|\boldsymbol{\sigma}_{i}-\tilde{\boldsymbol{\sigma}}_{h}\right\|+\left\|y_{i}-\tilde{u}_{h}\right\|_{L^{4}(\Omega)}\right) \leq C h^{k-\varepsilon} \delta .
$$

Using (4.12a)-4.12c), we note that for any $\left(v_{h}, \mathbf{w}_{h}, \boldsymbol{\tau}_{h}\right) \in V_{h} \times \mathbf{W}_{h} \times \mathbf{W}_{h}$,

$$
\begin{array}{r}
A\left(\mathbf{q}_{1}-\mathbf{q}_{2}, \mathbf{w}_{h}\right)-A_{1}\left(\mathbf{w}_{h}, y_{1}-y_{2}\right)+J_{1}\left(\boldsymbol{\sigma}_{1}-\boldsymbol{\sigma}_{2}, \mathbf{w}_{h}\right)=0, \\
A_{2}\left(u ; \mathbf{q}_{1}-\mathbf{q}_{2}, \boldsymbol{\tau}_{h}\right)+N\left(u, \mathbf{q} ; y_{1}-y_{2}, \boldsymbol{\tau}_{h}\right)-A\left(\boldsymbol{\sigma}_{1}-\boldsymbol{\sigma}_{2}, \boldsymbol{\tau}_{h}\right) \\
=N_{1}\left(z_{2}-u ; \boldsymbol{\theta}_{2}-\mathbf{q}, \boldsymbol{\tau}_{h}\right)+N_{2}\left(z_{2}-u, \mathbf{q}, \boldsymbol{\tau}_{h}\right) \\
-N_{1}\left(z_{1}-u ; \boldsymbol{\theta}_{1}-\mathbf{q}, \boldsymbol{\tau}_{h}\right)-N_{2}\left(z_{1}-u, \mathbf{q}, \boldsymbol{\tau}_{h}\right) \\
A_{1}\left(\boldsymbol{\sigma}_{1}-\boldsymbol{\sigma}_{2}, v_{h}\right)+J\left(y_{1}-y_{2}, v_{h}\right)=0 .
\end{array}
$$

We rewrite (4.43b) as

$$
\begin{aligned}
A_{2}\left(u ; \mathbf{q}_{1}-\mathbf{q}_{2}, \boldsymbol{\tau}_{h}\right)+N\left(u, \mathbf{q} ; y_{1}-y_{2}, \boldsymbol{\tau}_{h}\right)-A\left(\boldsymbol{\sigma}_{1}-\boldsymbol{\sigma}_{2}, \boldsymbol{\tau}_{h}\right) \\
=\int_{\Omega}\left(a\left(z_{1}\right)-a\left(z_{2}\right)\right)\left(\boldsymbol{\theta}_{1}-\mathbf{q}\right) \cdot \boldsymbol{\tau}_{h} d x \\
\quad-\int_{\Omega}\left(a\left(z_{2}\right)-a(u)\right)\left(\boldsymbol{\theta}_{1}-\boldsymbol{\theta}_{2}\right) \cdot \boldsymbol{\tau}_{h} d x \\
\quad+\int_{\Omega}\left(a\left(z_{1}\right)-a\left(z_{2}\right)-a_{u}\left(z_{2}\right)\left(z_{1}-z_{2}\right)\right) \mathbf{q} \cdot \boldsymbol{\tau}_{h} d x \\
\quad-\int_{\Omega}\left(a_{u}\left(z_{2}\right)-a_{u}(u)\right)\left(z_{1}-z_{2}\right) \mathbf{q} \cdot \boldsymbol{\tau}_{h} d x .
\end{aligned}
$$

Now using similar arguments as in the proof of Theorem 4.2, we easily obtain

$$
\begin{aligned}
\left(\| \mathbf{q}_{1}\right. & \left.-\mathbf{q}_{2} \|^{2}+J\left(y_{1}-y_{2}, y_{1}-y_{2}\right)+J_{1}\left(\boldsymbol{\sigma}_{1}-\boldsymbol{\sigma}_{2}, \boldsymbol{\sigma}_{1}-\boldsymbol{\sigma}_{2}\right)\right)^{\frac{1}{2}} \\
& \leq C_{1} h^{k-\varepsilon}\left(\left\|z_{1}-z_{2}\right\|_{L^{4}(\Omega)}+\left\|\boldsymbol{\theta}_{1}-\boldsymbol{\theta}_{2}\right\|\right)+C_{2}\left\|y_{1}-y_{2}\right\| \\
& \leq C_{1} h^{k-\varepsilon}\left\|\left(z_{1}, \boldsymbol{\theta}_{1}\right)-\left(z_{2}, \boldsymbol{\theta}_{2}\right)\right\|+C_{2}\left\|y_{1}-y_{2}\right\|
\end{aligned}
$$

and

$$
\left\|\boldsymbol{\sigma}_{1}-\boldsymbol{\sigma}_{2}\right\| \leq C_{1} h^{k-\varepsilon}\left\|\left(z_{1}, \boldsymbol{\theta}_{1}\right)-\left(z_{2}, \boldsymbol{\theta}_{2}\right)\right\|+C_{2}\left\|y_{1}-y_{2}\right\| .
$$

Then, an application of duality argument as in Lemma 4.4 yields

$$
\begin{aligned}
\left\|y_{1}-y_{2}\right\| \leq & C h^{\frac{1}{2}}\left(\max \left\{h, \frac{1}{\underline{C}_{11}}, h^{2} \bar{C}_{11}, \bar{C}_{22}\right\}\right)^{\frac{1}{2}}\left(\left\|\mathbf{q}_{1}-\mathbf{q}_{2}\right\|^{2}+J\left(y_{1}-y_{2}, y_{1}-y_{2}\right)\right. \\
& \left.+J_{1}\left(\boldsymbol{\sigma}_{1}-\boldsymbol{\sigma}_{2}, \boldsymbol{\sigma}_{1}-\boldsymbol{\sigma}_{2}\right)\right)^{\frac{1}{2}}+h^{k+\frac{1}{2}-\varepsilon}\left\|\left(z_{1}, \boldsymbol{\theta}_{1}\right)-\left(z_{2}, \boldsymbol{\theta}_{2}\right)\right\| .
\end{aligned}
$$

With an application of inverse inequality, we see that

$$
\left\|y_{1}-y_{2}\right\|_{L^{4}(\Omega)} \leq C h^{-\frac{1}{2}}\left\|y_{1}-y_{2}\right\| .
$$

We combine the estimates (4.45)-(4.48) to complete the rest of the proof. 
Now, we conclude that the map $S_{h}$ is a self-map on the ball $O_{\delta}\left(\tilde{u}_{h}, \tilde{\mathbf{q}}_{h}\right)$, and is continuous. Hence, an appeal to Brouwer fixed point theorem implies that $S_{h}$ has a fixed point, say $\left(u_{h}, \mathbf{q}_{h}\right) \in \mathcal{O}_{\delta}\left(\tilde{u}_{h}, \tilde{\mathbf{q}}_{h}\right)$, that is, $S_{h}\left(\left(u_{h}, \mathbf{q}_{h}\right)\right)=\left(u_{h}, \mathbf{q}_{h}\right)$ and $\boldsymbol{\sigma}_{h}$ can be easily obtained using (4.9b), $u_{h}$ and $\mathbf{q}_{h}$. Note that it is equivalent to proving existence of a unique triplet $\left(u_{h}, \mathbf{q}_{h}, \boldsymbol{\sigma}_{h}\right)$ of the problem (4.9a)-(4.9c) and hence, the triplet $\left(u_{h}, \mathbf{q}_{h}, \boldsymbol{\sigma}_{h}\right)$ is the unique solution of the problem (4.5a)-(4.5c).

Proof of Theorem 1.1. Note that the unique solution $\left(u_{h}, \mathbf{q}_{h}, \boldsymbol{\sigma}_{h}\right)$ of (4.9a)-(4.9c) satisfy, with the appropriate modification of (4.40), (4.27) and (4.21), the following estimates:

$$
\left(\left\|\mathbf{q}_{h}-\tilde{\mathbf{q}}_{h}\right\|^{2}+\int_{\Gamma} C_{11} \llbracket u_{h}-\tilde{u}_{h} \rrbracket^{2} d s+\int_{\Gamma_{I}} C_{22} \llbracket \boldsymbol{\sigma}_{h}-\tilde{\boldsymbol{\sigma}}_{h} \rrbracket^{2} d s\right)^{\frac{1}{2}} \leq C h^{k-\varepsilon} \delta,
$$

$\left\|u_{h}-\tilde{u}_{h}\right\| \leq C h^{\frac{1}{2}}\left(\max \left\{h, \frac{1}{\underline{C}_{11}}, h^{2} \bar{C}_{11}, \bar{C}_{22}\right\}\right)^{\frac{1}{2}}\left(\left\|\mathbf{q}_{h}-\tilde{\mathbf{q}}_{h}\right\|^{2}+\int_{\Gamma} C_{11} \llbracket u_{h}-\tilde{u}_{h} \rrbracket^{2} d s\right.$

$$
\begin{gathered}
\left.+\int_{\Gamma_{I}} C_{22} \llbracket \boldsymbol{\sigma}_{h}-\tilde{\boldsymbol{\sigma}}_{h} \rrbracket^{2} d s\right)^{\frac{1}{2}}+C h^{k+\frac{1}{2}-\varepsilon} \delta, \\
\left\|\boldsymbol{\sigma}_{h}-\tilde{\boldsymbol{\sigma}}_{h}\right\| \leq C\left(\left\|\mathbf{q}_{h}-\tilde{\mathbf{q}}_{h}\right\|+\left\|u_{h}-\tilde{u}_{h}\right\|+h^{k-\varepsilon} \delta\right) .
\end{gathered}
$$

Now, choosing $\varepsilon \in(0,1 / 2]$ and for $k \geq 1$, we obtain from (4.49)

$$
\left(\left\|\mathbf{q}_{h}-\tilde{\mathbf{q}}_{h}\right\|^{2}+\int_{\Gamma} C_{11} \llbracket u_{h}-\tilde{u}_{h} \rrbracket^{2} d s+\int_{\Gamma_{I}} C_{22} \llbracket \boldsymbol{\sigma}_{h}-\tilde{\boldsymbol{\sigma}}_{h} \rrbracket^{2} d s\right)^{\frac{1}{2}} \leq C h^{2 k} \leq C h^{k+1} .
$$

On substituting (4.52) in (4.50), we obtain with the choice $\varepsilon \in(0,1 / 2]$ and for $k \geq 1$,

$$
\left\|u_{h}-\tilde{u}_{h}\right\| \leq C h^{2 k} \leq C h^{k+1} .
$$

An application on (4.53) in (4.51) yields

$$
\left\|\boldsymbol{\sigma}_{h}-\tilde{\boldsymbol{\sigma}}_{h}\right\| \leq C h^{k+1} .
$$

Using triangle inequality, (4.49)-(4.51), and an application of Theorem 4.1 yields, for $k \geq 1$,

$$
\left\|u-u_{h}\right\|+\left\|\mathbf{q}-\mathbf{q}_{h}\right\|+\left\|\boldsymbol{\sigma}-\boldsymbol{\sigma}_{h}\right\| \leq C h^{k+1} .
$$

\section{Postprocessing of $u_{h}$ And SUPERCOnVERGEnCE RESUlts}

In this section, we discuss postprocessing $u_{h}^{*}$ of $u_{h}$ and then show that $\left\|u-u_{h}^{*}\right\|=$ $O\left(h^{k+2}\right)$.

On the element $K$, we define the new approximation $u_{h}^{*} \in \mathcal{P}^{k+1}(K)$ as

$$
u_{h}^{*}=u_{h}^{p}+\frac{1}{|K|} \int_{K} u_{h} d x,
$$

where $u_{h}^{p}$ is the polynomial in $\mathcal{P}_{0}^{k+1}(K)$ satisfying

$$
\left(a\left(u_{h}\right) \nabla u_{h}^{p}, \nabla v\right)_{K}=(f, v)_{K}+\left\langle\hat{\boldsymbol{\sigma}}_{h} \cdot \boldsymbol{\nu}, v\right\rangle_{\partial K}, \quad \forall v \in \mathcal{P}_{0}^{k+1}(K) .
$$

Here $\mathcal{P}_{0}^{k+1}(K)$ is the set of polynomials in $\mathcal{P}^{k+1}(K)$ with zero mean.

Note that compared to [10,4.1], we need to solve only a linear problem (5.2) on each element $K \in \mathcal{T}_{h}$.

We are now ready to state a superconvergence result. 
Theorem 5.1. Assume that $\Omega$ is bounded convex polygon domain in $\mathbb{R}^{2}$. Then for any method of the form (4.6a)-(4.6c) for $k \geq 1$, there exists a positive constant $C$ independent of $h$ such that

$$
\left\|u-u_{h}^{*}\right\| \leq C h^{k+2} .
$$

Below, we present a lemma similar to Lemma 3.6.

Lemma 5.1. For any method of the form (4.9a) (4.9c), the following equality holds:

$$
\begin{aligned}
T+\left\|a(u)^{\frac{1}{2}}\left(\boldsymbol{\Pi}_{k} \mathbf{q}-\mathbf{q}_{h}\right)\right\|^{2}= & \left(\boldsymbol{\Pi}_{k} \mathbf{q}-\mathbf{q}, \boldsymbol{\Pi}_{k} \boldsymbol{\sigma}-\boldsymbol{\sigma}_{h}\right)+A\left(u ; \boldsymbol{\Pi}_{k} \mathbf{q}-\mathbf{q}, \boldsymbol{\Pi}_{k} \mathbf{q}-\mathbf{q}_{h}\right) \\
& -\left(\boldsymbol{\Pi}_{k} \boldsymbol{\sigma}-\boldsymbol{\sigma}, \boldsymbol{\Pi}_{k} \mathbf{q}-\mathbf{q}_{h}\right)-N\left(u, \mathbf{q} ; u-u_{h}, \boldsymbol{\Pi}_{k} \mathbf{q}-\mathbf{q}_{h}\right) \\
& -T_{2}-T_{3}+N_{1}\left(u_{h}-u ; \mathbf{q}_{h}-\mathbf{q}, \boldsymbol{\Pi}_{k} \mathbf{q}-\mathbf{q}_{h}\right) \\
& +N_{2}\left(u_{h}-u ; \mathbf{q}, \boldsymbol{\Pi}_{k} \mathbf{q}-\mathbf{q}_{h}\right),
\end{aligned}
$$

where $T$ is defined by (3.52) and $T_{2}$ and $T_{3}$ are the same as defined in Lemma (3.6).

Proof. To prove (5.4), we now recall equations (4.9a)-(4.9c). Using the definition of the projections $\boldsymbol{\Pi}_{k}$ and $\mathbb{P}_{k}$, we rewrite the system (4.9a)-(4.9c) as

$$
\begin{aligned}
& \left(\boldsymbol{\Pi}_{k} \mathbf{q}-\mathbf{q}_{h}, \mathbf{w}_{h}\right)+\left(\mathbb{P}_{k} u-u_{h}, \nabla \cdot \mathbf{w}_{h}\right)-\left\langle u-\hat{u}_{h}, \mathbf{w}_{h} \cdot n\right\rangle \\
& =\left(\boldsymbol{\Pi}_{k} \mathbf{q}-\mathbf{q}, \mathbf{w}_{h}\right), \quad \forall \mathbf{w}_{h} \in \mathbf{W}_{h}, \\
& A_{2}\left(u ; \mathbf{q}-\mathbf{q}_{h}, \boldsymbol{\tau}_{h}\right)+N\left(u, \mathbf{q} ; u-u_{h}, \boldsymbol{\tau}_{h}\right)-\left(\boldsymbol{\Pi}_{k} \boldsymbol{\sigma}-\boldsymbol{\sigma}_{h}, \boldsymbol{\tau}_{h}\right) \\
& =-\left(\boldsymbol{\Pi}_{k} \boldsymbol{\sigma}-\boldsymbol{\sigma}, \boldsymbol{\tau}_{h}\right)+N_{1}\left(u_{h}-u ; \mathbf{q}_{h}-\mathbf{q}, \boldsymbol{\tau}_{h}\right) \\
& +N_{2}\left(u_{h}-u ; \mathbf{q}, \boldsymbol{\tau}_{h}\right), \quad \forall \boldsymbol{\tau}_{h} \in \mathbf{W}_{h}, \\
& \left(\boldsymbol{\Pi}_{k} \boldsymbol{\sigma}-\boldsymbol{\sigma}_{h}, \nabla v_{h}\right)-\left\langle\left(\boldsymbol{\sigma}-\hat{\boldsymbol{\sigma}}_{h}\right) \cdot \boldsymbol{\nu}, v_{h}\right\rangle=0, \quad \forall v_{h} \in V_{h} .
\end{aligned}
$$

Choose $\mathbf{w}_{h}=\boldsymbol{\Pi}_{k} \boldsymbol{\sigma}-\boldsymbol{\sigma}_{h}, \boldsymbol{\tau}_{h}=\boldsymbol{\Pi}_{k} \mathbf{q}-\mathbf{q}_{h}$ and $v_{h}=\mathbb{P}_{k} u-u_{h}$ in (5.5)-(5.7). Then add the resulting equations to obtain

$$
\begin{aligned}
&\left(a(u)\left(\boldsymbol{\Pi}_{k} \mathbf{q}-\mathbf{q}_{h}\right), \boldsymbol{\Pi}_{k} \mathbf{q}-\mathbf{q}_{h}\right) \\
&=\left(\boldsymbol{\Pi}_{k} \mathbf{q}-\mathbf{q}, \boldsymbol{\Pi}_{k} \boldsymbol{\sigma}-\boldsymbol{\sigma}_{h}\right)+A\left(u ; \boldsymbol{\Pi}_{k} \mathbf{q}-\mathbf{q}, \boldsymbol{\Pi}_{k} \mathbf{q}-\mathbf{q}_{h}\right) \\
&-\left(\boldsymbol{\Pi}_{k} \boldsymbol{\sigma}-\boldsymbol{\sigma}, \boldsymbol{\Pi}_{k} \mathbf{q}-\mathbf{q}_{h}\right)-N\left(u, \mathbf{q} ; u-u_{h}, \boldsymbol{\Pi}_{k} \mathbf{q}-\mathbf{q}_{h}\right) \\
&+\left\langle u_{h}-\mathbb{P}_{k} u,\left(\boldsymbol{\Pi}_{k} \boldsymbol{\sigma}-\boldsymbol{\sigma}_{h}\right) \cdot \boldsymbol{\nu}\right\rangle+\left\langle u-\hat{u}_{h},\left(\boldsymbol{\Pi}_{k} \boldsymbol{\sigma}-\boldsymbol{\sigma}_{h}\right) \cdot \boldsymbol{\nu}\right\rangle \\
&+\left\langle\left(\boldsymbol{\sigma}-\hat{\boldsymbol{\sigma}}_{h}\right) \cdot \boldsymbol{\nu}, \mathbb{P}_{k} u-u_{h}\right\rangle+N_{1}\left(u_{h}-u ; \mathbf{q}_{h}-\mathbf{q}, \boldsymbol{\Pi}_{k} \mathbf{q}-\mathbf{q}_{h}\right) \\
&+N_{2}\left(u_{h}-u ; \mathbf{q}, \boldsymbol{\Pi}_{k} \mathbf{q}-\mathbf{q}_{h}\right) \\
&=\left(\boldsymbol{\Pi}_{k} \mathbf{q}-\mathbf{q}, \boldsymbol{\Pi}_{k} \boldsymbol{\sigma}-\boldsymbol{\sigma}_{h}\right)+A\left(u ; \boldsymbol{\Pi}_{k} \mathbf{q}-\mathbf{q}, \boldsymbol{\Pi}_{k} \mathbf{q}-\mathbf{q}_{h}\right) \\
&-\left(\boldsymbol{\Pi}_{k} \boldsymbol{\sigma}-\boldsymbol{\sigma}, \boldsymbol{\Pi}_{k} \mathbf{q}-\mathbf{q}_{h}\right)-N\left(u, \mathbf{q} ; u-u_{h}, \boldsymbol{\Pi}_{k} \mathbf{q}-\mathbf{q}_{h}\right)+I \\
&+N_{1}\left(u_{h}-u ; \mathbf{q}_{h}-\mathbf{q}, \boldsymbol{\Pi}_{k} \mathbf{q}-\mathbf{q}_{h}\right)+N_{2}\left(u_{h}-u ; \mathbf{q}, \boldsymbol{\Pi}_{k} \mathbf{q}-\mathbf{q}_{h}\right),
\end{aligned}
$$

where

$$
\begin{aligned}
I:= & \left\langle u-\mathbb{P}_{k} u,\left(\boldsymbol{\Pi}_{k} \boldsymbol{\sigma}-\boldsymbol{\sigma}_{h}\right) \cdot \boldsymbol{\nu}\right\rangle+\left\langle u_{h}-\hat{u}_{h},\left(\boldsymbol{\Pi}_{k} \boldsymbol{\sigma}-\boldsymbol{\sigma}_{h}\right) \cdot \boldsymbol{\nu}\right\rangle \\
& +\left\langle\left(\boldsymbol{\sigma}-\hat{\boldsymbol{\sigma}}_{h}\right) \cdot \boldsymbol{\nu}, \mathbb{P}_{k} u-u_{h}\right\rangle .
\end{aligned}
$$

Proceeding in a similar way as in the proof of Lemma 3.6. we obtain

$$
I=-T-T_{2}-T_{3} \text {. }
$$

Note that $T_{1}=T$ as in Lemma 3.6. This completes the rest of the proof. 
Lemma 5.2. If $T$ and $\Theta_{k}$ are defined as in (3.52) and (3.36), respectively, then there is a positive constant $C$ such that

$$
T \leq C \Upsilon h^{2 k+1}
$$

provided (3.6) with (3.7) is satisfied and

$$
\Upsilon=|u|_{H^{k+1}\left(\Omega_{h}\right)}^{2}+|\mathbf{q}|_{\mathbf{H}^{k+1}\left(\Omega_{h}\right)}^{2}+|\boldsymbol{\sigma}|_{\mathbf{H}^{k+1}\left(\Omega_{h}\right)}^{2} .
$$

Proof. In Lemma 5.1, we apply the Cauchy-Schwarz inequality and the Young's inequality with kick back arguments to obtain

$$
\begin{aligned}
\frac{1}{2}\left\|a(u)^{\frac{1}{2}}\left(\mathbf{\Pi}_{k} \mathbf{q}-\mathbf{q}_{h}\right)\right\|^{2}+T & \leq C\left(\left\|\mathbf{\Pi}_{k} \mathbf{q}-\mathbf{q}\right\|^{2}+\left\|u-u_{h}\right\|^{2}+\frac{1}{h}\left\|u-u_{h}\right\|_{L^{4}(\Omega)}^{4}\right) \\
(5.11) & +C\left(\frac{1}{h}\left\|u-u_{h}\right\|_{L^{4}(\Omega)}^{2}\left\|\mathbf{q}-\mathbf{q}_{h}\right\|^{2}\right)+\left|T_{2}\right|+\left|T_{3}\right| .
\end{aligned}
$$

To complete the proof of (5.10), it is sufficient to estimate $\left|T_{2}\right|$ and $\left|T_{3}\right|$. Now substitute the expression of numerical traces in the definition of $T_{2}$ and $T_{3}$ and proceed in a similar manner as in the estimates of (3.67)-(3.69) to conclude

$$
\begin{array}{r}
\left|T_{2}\right| \leq \frac{1}{4} T+C\left(\bar{C}_{11}+\frac{1}{\underline{C}_{22}}\right) \sum_{i=1}^{N_{h}}\left\|u-\mathbb{P}_{k} u\right\|_{L^{2}\left(\partial K_{i}\right)}^{2}, \\
\left|T_{3}\right| \leq \frac{1}{4} T+C\left(\bar{C}_{22}+\frac{1}{\underline{C}_{11}}\right) \sum_{i=1}^{N_{h}}\left\|\left(P_{\partial} \boldsymbol{\sigma}-\Pi \boldsymbol{\Pi}\right) \cdot n\right\|_{L^{2}\left(\partial K_{i}\right)}^{2} .
\end{array}
$$

On substituting estimates (5.12)-(5.13) in (5.11) and using approximation properties of the projection $\Pi_{k}, \mathbb{P}_{k}$ and $P_{\partial}$ along with estimate (4.55), we arrive at

$$
T \leq C \Upsilon \max \left\{\bar{C}_{11}+\frac{1}{\underline{C}_{22}}, \bar{C}_{22}+\frac{1}{\underline{C}_{11}}\right\} h^{2 k+1},
$$

where $\Upsilon=|u|_{H^{k+1}\left(\Omega_{h}\right)}^{2}+|\mathbf{q}|_{\mathbf{H}^{k+1}\left(\Omega_{h}\right)}^{2}+|\boldsymbol{\sigma}|_{\mathbf{H}^{k+1}\left(\Omega_{h}\right)}^{2}$, this completes the rest of the proof.

Remark 5.1. Note that for LDG-H methods characterized by (3.8a)-(3.8b), we note that the results (3.64)-(3.65) of Lemma 5.2 are valid under conditions on $\tau$ discussed in Remark 3.1.

Below, we state a lemma which plays a crucial role in obtaining superconvergence estimate for $u_{h}^{*}$. This is related to the superconvergence of $P^{k-1}\left(u-u_{h}\right)$.

Lemma 5.3. Assume that $\Omega$ is such that the elliptic regularity (4.29) holds. Then for any method in the general form (4.6a)-(4.6c), there exits a positive constant $C$ independent of $h$ such that

$$
\left\|P^{k-1}\left(u-u_{h}\right)\right\| \leq C h^{k+2} .
$$

Proof. Setting $\Lambda:=\left(P^{k-1}\left(u-u_{h}\right), \theta\right)$, we note that using (4.30c) we get

$$
\begin{aligned}
\Lambda & =\left(P^{k-1}\left(u-u_{h}\right), \nabla \cdot \chi+a_{u}(u) \mathbf{q} \cdot \mathbf{p}\right) \\
& =\left(u-u_{h}, \nabla \cdot \Pi_{k-1}^{R T} \chi\right)+\left(P^{k-1}\left(u-u_{h}\right), a_{u}(u) \mathbf{q} \cdot \mathbf{p}\right) .
\end{aligned}
$$

Now, choose $\mathbf{w}_{h}=\Pi_{k-1}^{R T} \chi$ in 4.9a) to obtain

(5.16) $\Lambda=-\left(\mathbf{e}_{\mathbf{q}}, \Pi_{k-1}^{R T} \boldsymbol{\chi}\right)+\left\langle u-\hat{u}_{h}, \Pi_{k-1}^{R T} \boldsymbol{\chi} \cdot \boldsymbol{\nu}\right\rangle+\left(P^{k-1}\left(u-u_{h}\right), a_{u}(u) \mathbf{q} \cdot \mathbf{p}\right)$. 
Since $\Pi_{k-1}^{R T} \chi \in \mathbf{H}\left(\operatorname{div}, \Omega_{h}\right)$ and $u-\hat{u}_{h}=0$ on $\partial \Omega$ with $\left[u-\hat{u}_{h}\right]=0$, on $\Gamma_{I}$, we arrive at

$$
\left\langle u-\hat{u}_{h}, \Pi_{k-1}^{R T} \chi \cdot \nu\right\rangle=0 .
$$

Hence, the second term on the right-hand side of (5.16) vanishes.

Now, we obtain using (4.30b) in (5.16) and definitions of bilinear functionals $A, A_{2}$, $N, N_{1}$ and $N_{2}$

$$
\begin{aligned}
\Lambda= & A\left(\mathbf{e}_{\mathbf{q}}, \boldsymbol{\chi}-\Pi_{k-1}^{R T} \chi\right)+A_{2}\left(u ; \mathbf{e}_{\mathbf{q}}, \mathbf{p}\right)+N\left(u, \mathbf{q} ; P^{k-1}\left(u-u_{h}\right), \mathbf{p}\right) \\
= & A\left(\mathbf{e}_{\mathbf{q}}, \boldsymbol{\chi}-\Pi_{k-1}^{R T} \chi\right)+A_{2}\left(u ; \mathbf{e}_{\mathbf{q}}, \mathbf{p}-P^{k} \mathbf{p}\right)+A_{2}\left(u ; \mathbf{e}_{\mathbf{q}}, P^{k} \mathbf{p}\right) \\
& +N\left(u, \mathbf{q} ; P^{k-1}\left(u-u_{h}\right), \mathbf{p}\right) .
\end{aligned}
$$

Now, choose $\boldsymbol{\tau}_{h}=P^{k} \mathbf{p}$ in (4.9b) to arrive at

$$
\begin{aligned}
\Lambda= & A\left(\mathbf{e}_{\mathbf{q}}, \boldsymbol{\chi}-\Pi_{k-1}^{R T} \boldsymbol{\chi}\right)+A_{2}\left(u ; \mathbf{e}_{\mathbf{q}}, \mathbf{p}-P^{k} \mathbf{p}\right)-N\left(u ; \mathbf{q}\left(u-u_{h}\right), P^{k} \mathbf{p}\right) \\
& +\left(\mathbf{e}_{\boldsymbol{\sigma}}, P^{k} \mathbf{p}\right)+N_{1}\left(u_{h}-u ; \mathbf{q}_{h}-\mathbf{q}, P^{k} \mathbf{p}\right)+N_{2}\left(u_{h}-u ; \mathbf{q}, P^{k} \mathbf{p}\right) \\
& +N\left(u, \mathbf{q} ; P^{k-1}\left(u-u_{h}\right), \mathbf{p}\right) .
\end{aligned}
$$

Now, we rewrite (5.17) first and then use 4.30a to obtain

$$
\begin{aligned}
\Lambda= & A\left(\mathbf{e}_{\mathbf{q}}, \boldsymbol{\chi}-\Pi_{k-1}^{R T} \chi\right)+A_{2}\left(u ; \mathbf{e}_{\mathbf{q}}, \mathbf{p}-P^{k} \mathbf{p}\right)+A\left(\mathbf{e}_{\boldsymbol{\sigma}}, P^{k} \mathbf{p}-\mathbf{p}\right)+A\left(\mathbf{e}_{\boldsymbol{\sigma}}, \nabla \phi\right) \\
& +N\left(u, \mathbf{q} ;\left(u-u_{h}\right), \mathbf{p}-P^{k} \mathbf{p}\right)+N_{1}\left(u_{h}-u ; \mathbf{q}_{h}-\mathbf{q}, P^{k} \mathbf{p}\right) \\
& +N_{2}\left(u_{h}-u ; \mathbf{q}, P^{k} \mathbf{p}\right)+N_{1}\left(u, \mathbf{q} ; P^{k-1}\left(u-u_{h}\right)-\left(u-u_{h}\right), \mathbf{p}\right) \\
= & E_{1}+E_{2}+E_{3}+E_{4}+E_{5}+E_{6}+E_{7}+E_{8} .
\end{aligned}
$$

Let us estimate each of the terms $E_{1}, \cdots, E_{8}$ in (5.18). Since $k \geq 1$, we obtain using the Cauchy-Schwarz inequality and approximation properties of $\Pi_{k-1}^{R T}$ and $P^{k}$,

$$
\left|E_{1}+E_{2}+E_{3}+E_{5}\right| \leq C h\left(\left\|\mathbf{e}_{\mathbf{q}}\right\|+\left\|\mathbf{e}_{\boldsymbol{\sigma}}\right\|+\left\|e_{u}\right\|\right)\left(\|\chi\|_{\mathbf{H}^{1}(\Omega)}+\|\mathbf{p}\|_{\mathbf{H}^{1}(\Omega)}\right) .
$$

An application of Theorem 1.1 yields

$$
\left|E_{1}+E_{2}+E_{3}+E_{5}\right| \leq C h^{k+2}\left(\|\chi\|_{\mathbf{H}^{1}(\Omega)}+\|\mathbf{p}\|_{\mathbf{H}^{1}(\Omega)}\right) .
$$

To estimate $E_{4}$, we use (4.9c) to rewrite it as

$$
\begin{aligned}
E_{4} & =\left(\mathbf{e}_{\boldsymbol{\sigma}}, \nabla\left(\phi-P^{k} \phi\right)\right)+\left(\mathbf{e}_{\boldsymbol{\sigma}}, \nabla P^{k} \phi\right) \\
& =\left(\mathbf{e}_{\boldsymbol{\sigma}}, \nabla\left(\phi-P^{k} \phi\right)\right)+\left\langle\left(\boldsymbol{\sigma}-\hat{\boldsymbol{\sigma}}_{h}\right) \cdot \boldsymbol{\nu}, P^{k} \phi\right\rangle .
\end{aligned}
$$

Since $\llbracket \phi \rrbracket=0$ on $\Gamma_{I}$ and $\phi=0$ on boundary of $\Omega$, we rewrite the second term on the right-hand of (5.19) as

$$
\left\langle\left(\boldsymbol{\sigma}-\hat{\boldsymbol{\sigma}}_{h}\right) \cdot \boldsymbol{\nu}, P^{k} \phi\right\rangle=\int_{\Gamma}\left(\left\{\boldsymbol{\sigma}-\boldsymbol{\sigma}_{h}\right\}-\mathbf{C}_{12} \llbracket \boldsymbol{\sigma}-\boldsymbol{\sigma}_{h} \rrbracket-C_{11} \llbracket u-u_{h} \rrbracket\right) \llbracket \phi-P^{k} \phi \rrbracket d s,
$$

and hence, we obtain

$$
\begin{aligned}
E_{4}= & \left.\left(\mathbf{e}_{\boldsymbol{\sigma}}, \nabla\left(\phi-P^{k} \phi\right)\right)+\int_{\Gamma}\left(\left\{\boldsymbol{\sigma}-\boldsymbol{\sigma}_{h}\right\}\right\}-\mathbf{C}_{12} \llbracket \boldsymbol{\sigma}-\boldsymbol{\sigma}_{h} \rrbracket-C_{11} \llbracket u-u_{h} \rrbracket\right) \llbracket \phi-P^{k} \phi \rrbracket d s \\
\leq & C h\left\|\mathbf{e}_{\boldsymbol{\sigma}}\right\|\|\phi\|_{H^{2}(\Omega)}+C \sum_{K \in \mathcal{T}_{h}}\left\|\boldsymbol{\sigma}-\boldsymbol{\sigma}_{h}\right\|_{L^{2}(\partial K)}\left\|\phi-P^{k} \phi\right\|_{L^{2}(\partial K)} \\
& +C\left\{\max \left\{\frac{1}{C_{22}}, C_{11}\right\} T\right\}^{\frac{1}{2}}\left(\sum_{e \in \Gamma}\left\|\phi-P^{k} \phi\right\|_{L^{2}(e)}\right) .
\end{aligned}
$$


Using Theorem 1.1 and projection properties of $P^{k}$, we obtain

$$
\begin{aligned}
E_{4} & \leq C\left(h^{k+2}+h^{k+\frac{5}{2}}\right)\|\phi\|_{H^{2}} \\
& \leq C h^{k+2}\|\phi\|_{H^{2}(\Omega)} .
\end{aligned}
$$

For $E_{6}$, an application of generalized Hölder's inequality with $\left\|P^{k} \mathbf{p}\right\|_{L^{4}(\Omega)} \leq$ $\|\mathbf{p}\|_{\mathbf{H}^{1}(\Omega)}$ and Theorem 1.1 yields, for $k \geq 1$,

$$
\begin{aligned}
\left|E_{6}\right| & \leq C\left\|u_{h}-u\right\|_{L^{4}(\Omega)}\left\|\mathbf{q}_{h}-\mathbf{q}\right\|\left\|P^{k} \mathbf{p}\right\|_{L^{4}(\Omega)} \\
& \leq C h^{k+\frac{1}{2}} h^{k+1}\|\mathbf{p}\|_{\mathbf{H}^{1}\left(\Omega_{h}\right)} \leq C h^{k+2}\|\mathbf{p}\|_{\mathbf{H}^{1}(\Omega)} .
\end{aligned}
$$

Similarly, for $k \geq 1$ and by Theorem 1.1, we obtain an estimate for $E_{7}$ as

$$
\begin{aligned}
\left|E_{7}\right| & \leq C\left\|u_{h}-u\right\|_{L^{4}(\Omega)}^{2}\left\|P^{k} \mathbf{p}\right\|_{L^{4}(\Omega)} \\
& \leq C h^{2 k+1}\|\mathbf{p}\|_{\mathbf{H}^{1}\left(\Omega_{h}\right)} \leq C h^{k+2}\|\mathbf{p}\|_{\mathbf{H}^{1}(\Omega)} .
\end{aligned}
$$

To estimate $E_{8}$, we use properties of $P^{k-1}$ and Theorem 1.1, to find that

$$
\begin{aligned}
E_{8} & =\left(P^{k-1}\left(u-u_{h}\right)-\left(u-u_{h}\right), a_{u}(u) \mathbf{q} \cdot \mathbf{p}-P^{k-1}\left(a_{u}(u) \mathbf{q} \cdot \mathbf{p}\right)\right) \\
& \leq C h\left\|u-u_{h}\right\|\|\mathbf{p}\|_{H^{1}(\Omega)} \leq C h^{k+2}\|\mathbf{p}\|_{H^{1}(\Omega)} .
\end{aligned}
$$

On combining the estimates (5.18) and using the elliptic regularity (4.29), we arrive at

$$
\Lambda \leq C h^{k+2}\|\theta\|
$$

With $\theta=P^{k-1}\left(u-u_{h}\right)$, we obtain the desired estimate (5.15) and this completes the proof.

Remark 5.2. As in [15], it is possible to show the following super approximation property:

$$
\left|P_{\partial}^{k}\left(u-\hat{u}_{h}\right)\right|_{L^{2}(\Gamma ; h)} \leq C h^{k+2} .
$$

Since the proof technique follows on similar lines of Lemma 4.3 of [15, we prefer not to repeat it here.

Proof of Theorem 5.1. By the definition of $u_{h}^{*}$ given in (5.1) on any element $K \in \mathcal{T}_{h}$, we note that

$$
\begin{aligned}
u-u_{h}^{*} & =u-u_{h}^{p}-\frac{1}{|K|} \int_{K} u_{h} d x \\
& =u-\frac{1}{|K|} \int_{K} u d x-u_{h}^{p}+\frac{1}{|K|} \int_{K}\left(u-u_{h}\right) d x \\
& =u^{p}-u_{h}^{p}+\frac{1}{|K|} \int_{K}\left(u-u_{h}\right) d x
\end{aligned}
$$

where $u^{p}$ stands for $u$ minus its average on $K$. Let $\mathcal{P}^{0}(K)$ denote the space of constant functions. For any $\phi \in L^{2}(K)$, let $P^{0} \phi \in \mathcal{P}^{0}(K)$ satisfy

$$
\left(\phi-P^{0} \phi, v\right)_{K}=0, \quad \forall v \in \mathcal{P}^{0}(K) .
$$

Note that, for any $\phi \in L^{2}(K), P^{0}$ satisfies

$$
P^{0} \phi=\frac{1}{K} \int_{K} \phi d x .
$$

We can easily observe that

$$
\left\|P^{0} \phi\right\|_{L^{2}(K)} \leq C\left\|P^{k-1} \phi\right\|_{L^{2}(K)},
$$


provided $k \geq 1$. By the definition of $P^{0}$, we now arrive at

$$
\begin{aligned}
\left\|u-u_{h}^{*}\right\|_{L^{2}(K)} & \leq\left\|P^{0}\left(u-u_{h}\right)\right\|_{L^{2}(K)}+\left\|u^{p}-u_{h}^{p}\right\|_{L^{2}(K)} \\
& \leq\left\|P^{k-1}\left(u-u_{h}\right)\right\|_{L^{2}(K)}+\left\|u^{p}-u_{h}^{p}\right\|_{L^{2}(K)},
\end{aligned}
$$

since $k-1 \geq 0$. An application of Lemma 5.3 yields

$$
\begin{aligned}
\left\|u-u_{h}^{*}\right\|_{L^{2}(\Omega)}^{2} & =\sum_{K \in \mathcal{T}_{h}}\left\|u-u_{h}^{*}\right\|_{L^{2}(K)}^{2} \\
& \leq C h^{2(k+2)}+\left\|u^{p}-u_{h}^{p}\right\|_{L^{2}(K)}^{2} .
\end{aligned}
$$

Suppose we estimate the last term on the right-hand side of (5.31) by

$$
\left\|u^{p}-u_{h}^{p}\right\|_{L^{2}(\Omega)}^{2}=\sum_{K \in \mathcal{T}_{h}}\left\|u^{p}-u_{h}^{p}\right\|_{L^{2}(K)}^{2} \leq C h^{2(k+2)},
$$

then substituting (5.32) in (5.31), we complete the rest of the proof.

In order to complete the proof of Theorem 5.1, it remains to derive (5.32).

Lemma 5.4. Let $\left.u^{p}\right|_{K}$ be defined as u minus its average on each $K$ and let $u_{h}^{p}$ be defined as in (5.2). Then there exists a positive constant $C$ such that

$$
\left\|u^{p}-u_{h}^{p}\right\|_{L^{2}(\Omega)}^{2}=\sum_{K \in \mathcal{T}_{h}}\left\|u^{p}-u_{h}^{p}\right\|_{L^{2}(K)}^{2} \leq C h^{2(k+2)},
$$

Proof. Since, by Poincaré inequality (see [5]), we note that

$$
\left\|u^{p}-u_{h}^{p}\right\|_{L^{2}(K)} \leq C h_{K}\left\|\nabla\left(u^{p}-u_{h}^{p}\right)\right\|_{L^{2}(K)},
$$

it is, therefore, enough to estimate the error in the gradient. Using the definition of $u_{h}^{p}$ given in (5.2), we note that, for any $v \in P_{0}^{k+1}(K)$,

$$
\begin{aligned}
\left(a\left(u_{h}\right) \nabla\left(u^{p}-u_{h}^{p}\right), \nabla v\right)_{K}= & \left(a\left(u_{h}\right) \nabla u, \nabla v\right)_{K}-(f, v)_{K}-\left\langle\hat{\boldsymbol{\sigma}}_{h} \cdot \boldsymbol{\nu}, v\right\rangle_{\partial K} \\
= & \left(\left(a\left(u_{h}\right)-a(u)\right) \mathbf{q}, \nabla v\right)_{K}+(\boldsymbol{\sigma}, \nabla v)_{K}-(f, v)_{K} \\
& -\left\langle\hat{\boldsymbol{\sigma}}_{h} \cdot \boldsymbol{\nu}, v\right\rangle_{\partial K},
\end{aligned}
$$

and now use Taylor expansion (4.3)-(4.4) for the first term and integration by parts for the second term on the right-hand side of (5.34) to arrive at

$$
\begin{aligned}
\left(a\left(u_{h}\right) \nabla\left(u^{p}-u_{h}^{p}\right), \nabla v\right)_{K}= & \left(a_{u} \mathbf{q}\left(u_{h}-u\right), \nabla v\right)_{K}+\left(\tilde{a}_{u u} \mathbf{q}\left(u_{h}-u\right)^{2}, \nabla v\right)_{K} \\
& +\left\langle\left(\boldsymbol{\sigma}-\hat{\boldsymbol{\sigma}}_{h}\right) \cdot \boldsymbol{\nu}, v\right\rangle_{\partial K} .
\end{aligned}
$$

Now using equation (4.9b), we obtain

$$
\begin{aligned}
\left(a\left(u_{h}\right) \nabla\left(u^{p}-u_{h}^{p}\right), \nabla v\right)_{K}= & \left(a(u) \mathbf{e}_{\mathbf{q}}, \nabla v\right)_{K}-\left(\tilde{a}_{u} \mathbf{e}_{\mathbf{q}} e_{u}, \nabla v\right)_{K}-\left(\mathbf{e}_{\boldsymbol{\sigma}}, \nabla v\right)_{K} \\
& +\left\langle\left(\boldsymbol{\sigma}-\hat{\boldsymbol{\sigma}}_{h}\right) \cdot \boldsymbol{\nu}, v\right\rangle_{\partial K} \\
= & I_{1}+I_{2}+I_{3}+I_{4} .
\end{aligned}
$$

Apply the Cauchy-Schwarz inequality to bound the first term as

$$
I_{1} \leq C\left\|\mathbf{e}_{\mathbf{q}}\right\|_{L^{2}(K)}\|\nabla v\|_{L^{2}(K)} .
$$

Again, using the generalized Hölder's inequality with Lemma 4.1, the inverse inequalities (2.3) and (4.53) yield

$$
\begin{aligned}
I_{2} & \leq C\left\|e_{u}\right\|\left\|_{L^{4}(K)}\right\| \mathbf{e}_{\mathbf{q}}\left\|_{L^{2}(K)}\right\| \nabla v \|_{L^{4}(K)} \\
& \leq C\left(\left\|u-\tilde{u}_{h}\right\|_{L^{4}(K)}+\left\|\tilde{u}_{h}-u_{h}\right\|_{L^{4}(K)}\right)\left\|\mathbf{e}_{\mathbf{q}}\right\|_{L^{2}(K)} h_{K}^{-\frac{1}{2}}\|\nabla v\|_{L^{2}(K)} \\
& \leq C h^{k}\left\|\mathbf{e}_{\mathbf{q}}\right\|_{L^{2}(K)}\|\nabla v\|_{L^{2}(K)} .
\end{aligned}
$$


Use integration by parts to rewrite the third term on the right-hand side of (5.35) as

$$
I_{3}=\left(\nabla \cdot \mathbf{e}_{\boldsymbol{\sigma}}, v\right)_{K}-\left\langle\mathbf{e}_{\boldsymbol{\sigma}} \cdot \boldsymbol{\nu}, v\right\rangle_{\partial K}
$$

Thus,

$$
\begin{aligned}
I_{3}+I_{4}= & \left(\nabla \cdot\left(\boldsymbol{\sigma}-\Pi_{k}^{R T} \boldsymbol{\sigma}\right), v\right)_{K}+\left(\nabla \cdot\left(\Pi_{k}^{R T} \boldsymbol{\sigma}-\boldsymbol{\sigma}_{h}\right), v\right)_{K} \\
& +\left\langle\left(\boldsymbol{\sigma}_{h}-\hat{\boldsymbol{\sigma}}_{h}\right) \cdot \boldsymbol{\nu}, v\right\rangle_{\partial K} .
\end{aligned}
$$

Using the definition of $\Pi_{k}^{R T}$, the Cauchy-Schwarz inequality, and the inverse estimates, we arrive at

$$
\begin{aligned}
I_{3}+I_{4} \leq & \left(\left\|f-P^{k} f\right\|_{L^{2}(K)}+h_{K}^{-1}\left\|\boldsymbol{\sigma}-\boldsymbol{\sigma}_{h}\right\|_{L^{2}(K)}\right. \\
& \left.+h_{K}^{-\frac{1}{2}}\left\|\left(\boldsymbol{\sigma}_{h}-\hat{\boldsymbol{\sigma}}_{h}\right) \cdot \boldsymbol{\nu}\right\|_{L^{2}(\partial K)}\right)\|v\|_{L^{2}(K)} .
\end{aligned}
$$

Using Poincaré inequality (see [5]), yields

$$
\begin{aligned}
I_{3}+I_{4} \leq & C\left(h_{K}\left\|f-P^{k} f\right\|_{L^{2}(K)}+\left\|\mathbf{e}_{\boldsymbol{\sigma}}\right\|_{L^{2}(K)}\right. \\
& \left.+h_{K}^{\frac{1}{2}}\left\|\left(\boldsymbol{\sigma}_{h}-\hat{\boldsymbol{\sigma}}_{h}\right) \cdot \boldsymbol{\nu}\right\|_{L^{2}(\partial K)}\right)\|\nabla v\|_{L^{2}(K)} .
\end{aligned}
$$

On substituting the estimates (5.36), (5.37) and (5.38) in (5.35), we obtain

$$
\begin{aligned}
\left(a\left(u_{h}\right) \nabla\left(u^{p}-u_{h}^{p}\right), \nabla v\right)_{K} \leq & C\left(\left\|\mathbf{e}_{\mathbf{q}}\right\|_{L^{2}(K)}+h_{K}\left\|f-P^{k} f\right\|_{L^{2}(K)}+\left\|\mathbf{e}_{\boldsymbol{\sigma}}\right\|_{L^{2}(K)}\right. \\
& \left.+h_{K}^{\frac{1}{2}}\left\|\left(\boldsymbol{\sigma}_{h}-\hat{\boldsymbol{\sigma}}_{h}\right) \cdot \boldsymbol{\nu}\right\|_{L^{2}(\partial K)}\right)\|\nabla v\|_{L^{2}(K)} .
\end{aligned}
$$

Now we note, using bounded below property of $a$, that

$$
\begin{aligned}
\alpha_{0}\left\|\nabla\left(P^{k+1} u^{p}-u_{h}^{p}\right)\right\|_{L^{2}(K)}^{2} \leq & \left(a\left(u_{h}\right) \nabla\left(P^{k+1} u^{p}-u_{h}^{p}\right), \nabla\left(P^{k+1} u^{p}-u_{h}^{p}\right)\right) \\
= & \left(a\left(u_{h}\right) \nabla\left(P^{k+1} u^{p}-u^{p}\right), \nabla\left(P^{k+1} u^{p}-u_{h}^{p}\right)\right) \\
& +\left(a\left(u_{h}\right) \nabla\left(u^{p}-u_{h}^{p}\right), \nabla\left(P^{k+1} u^{p}-u_{h}^{p}\right)\right) .
\end{aligned}
$$

Using the Cauchy-Schwarz inequality and (5.39) and replacing $v$ by $P^{k+1} u^{p}-u_{h}^{p}$, we arrive at

$$
\begin{aligned}
\left\|\nabla\left(P^{k+1} u^{p}-u_{h}^{p}\right)\right\|_{L^{2}(K)} \leq & C\left(\left\|\nabla\left(P^{k+1} u^{p}-u^{p}\right)\right\|_{L^{2}(K)}+\left\|\mathbf{e}_{\mathbf{q}}\right\|_{L^{2}(K)}\right. \\
& +h_{K}\left\|f-P^{k} f\right\|_{L^{2}(K)}+\left\|\mathbf{e}_{\boldsymbol{\sigma}}\right\|_{L^{2}(K)} \\
& \left.+h_{K}^{\frac{1}{2}}\left\|\left(\boldsymbol{\sigma}_{h}-\hat{\boldsymbol{\sigma}}_{h}\right) \cdot \boldsymbol{\nu}\right\|_{L^{2}(\partial K)}\right) .
\end{aligned}
$$

Finally, an application of triangle inequality yields

$$
\begin{gathered}
\left\|\nabla\left(u^{p}-u_{h}^{p}\right)\right\|_{L^{2}(K)} \leq\left\|\nabla\left(P^{k+1} u^{p}-u^{p}\right)\right\|_{L^{2}(K)}+\left\|\nabla\left(P^{k+1} u^{p}-u_{h}^{p}\right)\right\|_{L^{2}(K)} \\
\leq C\left(\left\|\nabla\left(P^{k+1} u^{p}-u^{p}\right)\right\|_{L^{2}(K)}+\left\|\mathbf{e}_{\mathbf{q}}\right\|_{L^{2}(K)}+h_{K}\left\|f-P^{k} f\right\|_{L^{2}(K)}\right. \\
\left.+\left\|\mathbf{e}_{\boldsymbol{\sigma}}\right\|_{L^{2}(K)}+h_{K}^{\frac{1}{2}}\left\|\left(\boldsymbol{\sigma}_{h}-\hat{\boldsymbol{\sigma}}_{h}\right) \cdot \boldsymbol{\nu}\right\|_{L^{2}(\partial K)}\right) .
\end{gathered}
$$

It is easy to note that

$$
\sum_{i=1}^{N_{h}} h_{i}^{\frac{1}{2}}\left\|\left(\boldsymbol{\sigma}_{h}-\hat{\boldsymbol{\sigma}}_{h}\right) \cdot \boldsymbol{\nu}\right\|_{L^{2}\left(\partial K_{i}\right)}^{2} \leq C h \max \left\{\bar{C}_{11}, \frac{1}{\underline{C}_{22}}\right\} T .
$$


An application of Lemma 5.2 yields

$$
\sum_{i=1}^{N_{h}} h_{i}\left\|\left(\boldsymbol{\sigma}_{h}-\hat{\boldsymbol{\sigma}}_{h}\right) \cdot \boldsymbol{\nu}\right\|_{L^{2}\left(\partial K_{i}\right)}^{2} \leq C h^{k+2} .
$$

Substitute (5.41) in (5.33), sum over all elements $K \in \mathcal{T}_{h}$, use Theorem 1.1 and (5.43) to arrive at

$$
\left\|u^{p}-u_{h}^{p}\right\| \leq C h^{k+2} .
$$

This completes the rest of the proof.

Remark 5.3. Note that error estimates obtained in Theorem 1.1 for quasilinear elliptic problems are exactly the same as in Theorem 3.2 for the nonselfadjoint elliptic problem and also estimates obtained for selfadjoint linear problems in [15].

Remark 5.4. In the proof of Lemma 4.2 and in the subsequent results in the Section 4 , we have assumed that the range of $\frac{\partial^{i} a}{\partial u^{i}}(x, v), x \in \bar{\Omega}, v \in \mathbb{R}, i=0,1,2$, is a compact set $[m, M] \subset \mathbb{R}$. But, we note that asymptotically only the values of $v \in\left[m_{u}-\delta^{*}, M_{u}+\delta^{*}\right] \subset \mathbb{R}$, where $0<\delta^{*}<1, m_{u}=\min \{u(x): x \in \bar{\Omega}\}$ and $M_{u}=$ $\max \{u(x): x \in \bar{\Omega}\}$ are considered to derive the Lemma 4.2 and the subsequent results. To be more precise, the terms $\tilde{a}_{u}(z)$ and $\tilde{a}_{u u}(z),(z, \boldsymbol{\theta}) \in \mathcal{O}_{\delta}\left(\tilde{u}_{h}, \tilde{\mathbf{q}}_{h}\right)$ in (4.17) and (4.19) can be estimated as follows. Using inverse inequality (see [11, p. 140]), we obtain

$$
\left\|z-\tilde{u}_{h}\right\|_{L^{\infty}\left(K_{i}\right)} \leq C h_{i}^{-\frac{1}{2}}\left\|z-\tilde{u}_{h}\right\|_{L^{4}\left(K_{i}\right)} .
$$

Since $(z, \boldsymbol{\theta}) \in \mathcal{O}\left(\tilde{u}_{h}, \tilde{\mathbf{q}}_{h}\right)$, we find using (5.45), Theorem 4.1 and the definition of $\delta$, (4.14), that

$$
\begin{aligned}
\|z-u\|_{L^{\infty}(\Omega)} & \leq\left\|z-\tilde{u}_{h}\right\|_{L^{\infty}(\Omega)}+\left\|\tilde{u}_{h}-u\right\|_{L^{\infty}(\Omega)} \\
& \leq C \sum_{i=1}^{N_{h}} h_{i}^{-\frac{1}{2}}\left\|z-\tilde{u}_{h}\right\|_{L^{4}\left(K_{i}\right)}+\left\|u-I_{h} u\right\|_{L^{\infty}(\Omega)}+\left\|I_{h} u-\tilde{u}_{h}\right\|_{L^{\infty}(\Omega)} \\
& \leq C h^{-\frac{1}{2}} \delta+C h^{k}\|u\|_{H^{k+1}\left(\Omega, \mathcal{T}_{h}\right)}+C \sum_{i=1}^{N_{h}} h_{i}^{-\frac{1}{2}}\left\|I_{h} u-\tilde{u}_{h}\right\|_{L^{4}(\Omega)} \\
& \leq C h^{k-\varepsilon}+C h^{k} \leq C h^{k-\varepsilon} .
\end{aligned}
$$

Therefore, for sufficiently small $h,\|z\|_{L^{\infty}(\Omega)} \leq \delta^{*}+\|u\|_{L^{\infty}(\Omega)}$, where $0<\delta<1$. Now since the nonlinear functions $a_{u}$ and $a_{u u}$ are continuous, they map the compact set $\left[m_{u}-\delta^{*}, M_{u}+\delta^{*}\right]$ to a compact set in $\mathbb{R}$ and hence, the results in Lemma 4.16 and the subsequent results in Section 3 remain valid when $a(z), a_{u}(z), a_{u u}(z)$ are bounded for bounded $u$.

\section{NumericAl EXPERIMENTS}

In this section, we discuss some numerical results to illustrate the performance of the LDG method applied to a nonlinear elliptic problem. Since the scheme deals with discontinuous finite element spaces, the global basis functions can have support only on a single finite element. Hence, the assembly of the local matrices to the corresponding global matrices is easier than in the case of conforming finite element methods. For the example, we take $\Omega=(0,1) \times(0,1)$. The finite element subdivision $\mathcal{T}_{h}$ is of uniform triangles and the discontinuous finite element spaces 
TABLE 1. History of Convergence for $\tau \equiv 1$

\begin{tabular}{|c|c|cc|cc|cc|}
\hline & mesh & $\left\|u-u_{h}\right\|$ & & $\left\|\boldsymbol{\sigma}-\boldsymbol{\sigma}_{h}\right\|$ & & $\left\|u-u_{h}^{*}\right\|$ & \\
\hline $\mathrm{k}$ & $\mathrm{h}$ & error & order & error & order & error & order \\
\hline \multirow{4}{*}{1} & $1 / 3$ & $0.84 \mathrm{e}-2$ & - & $0.25 \mathrm{e}-1$ & - & $0.16 \mathrm{e}-2$ & - \\
& $1 / 5$ & $0.23 \mathrm{e}-2$ & 1.86 & $0.68 \mathrm{e}-2$ & 1.88 & $0.23 \mathrm{e}-3$ & 2.78 \\
& $1 / 7$ & $0.10 \mathrm{e}-2$ & 1.97 & $0.31 \mathrm{e}-2$ & 1.96 & $0.73 \mathrm{e}-4$ & 2.87 \\
& $1 / 9$ & $0.58 \mathrm{e}-3$ & 2.00 & $0.17 \mathrm{e}-2$ & 1.97 & $0.32 \mathrm{e}-4$ & 2.90 \\
& $1 / 11$ & $0.37 \mathrm{e}-3$ & 2.00 & $0.11 \mathrm{e}-2$ & 1.98 & $0.16 \mathrm{e}-4$ & 2.92 \\
& $1 / 13$ & $0.26 \mathrm{e}-3$ & 2.01 & $0.78 \mathrm{e}-3$ & 1.99 & $0.97 \mathrm{e}-5$ & 2.94 \\
\hline \multirow{4}{*}{2} & $1 / 3$ & $0.17 \mathrm{e}-2$ & - & $0.45 \mathrm{e}-2$ & - & $0.38 \mathrm{e}-3$ & - \\
& $1 / 5$ & $0.21 \mathrm{e}-3$ & 3.02 & $0.57 \mathrm{e}-3$ & 2.98 & $0.24 \mathrm{e}-4$ & 3.97 \\
& $1 / 7$ & $0.62 \mathrm{e}-4$ & 3.04 & $0.17 \mathrm{e}-3$ & 2.99 & $0.48 \mathrm{e}-5$ & 3.99 \\
& $1 / 9$ & $0.26 \mathrm{e}-4$ & 3.03 & $0.72 \mathrm{e}-4$ & 2.99 & $0.15 \mathrm{e}-5$ & 4.00 \\
& $1 / 11$ & $0.13 \mathrm{e}-4$ & 3.03 & $0.37 \mathrm{e}-4$ & 2.99 & $0.62 \mathrm{e}-6$ & 4.01 \\
& $1 / 13$ & $0.76 \mathrm{e}-5$ & 3.02 & $0.21 \mathrm{e}-4$ & 2.99 & $0.30 \mathrm{e}-6$ & 4.01 \\
\hline
\end{tabular}

of degree $k=1$ and $k=2\left(k_{i}=k, \forall i\right)$. The LDG method (4.5a)-(4.5c) has three unknowns; namely, $u_{h}, \mathbf{q}_{h}$ and $\boldsymbol{\sigma}_{h}$. Using (4.5a), we first solve $\mathbf{q}_{h}$ in terms of $u_{h}$ to write the system (4.5b)-(4.5c) in two unknowns $u_{h}$ and $\boldsymbol{\sigma}_{h}$. Then, we apply Newton's method to solve the resulting nonlinear system.

Let $N$ and $M$ be the dimensions of $V_{h}$ and $W_{h}$. Now, let $\left\{\chi_{l}\right\}_{l=1}^{M}$ denote bases for $\mathbf{W}_{h}$, which is obtained by taking the tensor product of the basis of $V_{h}$. Then, we define the following matrices:

$$
\begin{array}{ll}
A=\left[A_{m l}\right]_{1 \leq m, l \leq M}, & B=\left[B_{l i}\right]_{1 \leq l \leq M, 1 \leq i \leq N}, \\
J=\left[J_{i j}\right]_{1 \leq i, j \leq N}, & K=\left[K_{l m}\right]_{1 \leq l, m \leq M},
\end{array}
$$

and the vector

$$
L=\left[L_{i}\right]_{1 \leq i \leq N, 1}
$$

where

$$
\begin{aligned}
& A_{m l}=\int_{\Omega} \chi_{m} \cdot \chi_{l} d x, \quad J_{i j}=\sum_{e_{k} \in \Gamma} \int_{e_{k}} C_{11} \llbracket \phi_{i} \rrbracket \llbracket \phi_{j} \rrbracket d s, \\
& \left.B_{l i}=\sum_{i=1}^{N_{h}} \int_{K_{i}} \phi_{i} \nabla \cdot \chi_{l} d x-\int_{\Gamma_{I}}\left(\left\{\phi_{i}\right\}\right\}+\mathbf{C}_{12} \llbracket \phi_{i} \rrbracket\right) \llbracket \chi_{l} \rrbracket d s, \\
& K_{l m}=\sum_{e_{k} \in \Gamma_{I}} \int_{e_{k}} C_{22} \llbracket \chi_{l} \rrbracket \llbracket \chi_{m} \rrbracket d s,
\end{aligned}
$$

Write

$$
u_{h}=\sum_{i=1}^{N} \alpha_{i} \phi_{i}, \mathbf{q}_{h}=\sum_{l=1}^{M} \beta_{l} \boldsymbol{\chi}_{l} \text { and } \boldsymbol{\sigma}_{h}=\sum_{l=1}^{M} \gamma_{l} \boldsymbol{\chi}_{l},
$$

where $\boldsymbol{\alpha}=\left[\alpha_{1}, \alpha_{2}, \cdots, \alpha_{N}\right], \boldsymbol{\beta}=\left[\beta_{1}, \beta_{2}, \cdots, \beta_{M}\right]$ and $\boldsymbol{\gamma}=\left[\gamma_{1}, \gamma_{2}, \cdots, \gamma_{M}\right]$. Using the bases for $V_{h}$ and $\mathbf{W}_{h}$, 4.5a an be reduced to the following matrix equation:

$$
A \boldsymbol{\beta}+B \boldsymbol{\alpha}+K \boldsymbol{\gamma}=0 .
$$


TABLE 2. History of Convergence for $\tau=O(h)$

\begin{tabular}{|c|c|cc|cc|cc|}
\hline & mesh & $\left\|u-u_{h}\right\|$ & & $\left\|\boldsymbol{\sigma}-\boldsymbol{\sigma}_{h}\right\|$ & & $\left\|u-u_{h}^{*}\right\|$ & \\
\hline $\mathrm{k}$ & $\mathrm{l}$ & error & order & error & order & error & order \\
\hline \multirow{4}{*}{1} & $1 / 3$ & $0.15 \mathrm{e}-1$ & - & $0.25 \mathrm{e}-1$ & - & $0.15 \mathrm{e}-2$ & - \\
& $1 / 5$ & $0.79 \mathrm{e}-2$ & 0.92 & $0.66 \mathrm{e}-2$ & 1.89 & $0.21 \mathrm{e}-3$ & 2.85 \\
& $1 / 7$ & $0.52 \mathrm{e}-2$ & 1.03 & $0.30 \mathrm{e}-2$ & 1.96 & $0.65 \mathrm{e}-4$ & 2.95 \\
& $1 / 9$ & $0.38 \mathrm{e}-2$ & 1.04 & $0.17 \mathrm{e}-2$ & 1.98 & $0.27 \mathrm{e}-4$ & 2.97 \\
& $1 / 11$ & $0.30 \mathrm{e}-2$ & 1.04 & $0.11 \mathrm{e}-2$ & 1.98 & $0.14 \mathrm{e}-4$ & 2.98 \\
& $1 / 13$ & $0.25 \mathrm{e}-2$ & 1.04 & $0.76 \mathrm{e}-3$ & 1.99 & $0.82 \mathrm{e}-5$ & 2.99 \\
\hline \multirow{4}{*}{2} & $1 / 3$ & $0.31 \mathrm{e}-2$ & - & $0.44 \mathrm{e}-2$ & - & $0.28 \mathrm{e}-2$ & - \\
& $1 / 5$ & $0.69 \mathrm{e}-3$ & 2.15 & $0.56 \mathrm{e}-3$ & 3.00 & $0.13 \mathrm{e}-3$ & 4.44 \\
& $1 / 7$ & $0.29 \mathrm{e}-3$ & 2.10 & $0.16 \mathrm{e}-3$ & 3.00 & $0.22 \mathrm{e}-4$ & 4.30 \\
& $1 / 9$ & $0.16 \mathrm{e}-3$ & 2.07 & $0.69 \mathrm{e}-4$ & 3.00 & $0.67 \mathrm{e}-5$ & 4.20 \\
& $1 / 11$ & $0.10 \mathrm{e}-3$ & 2.06 & $0.36 \mathrm{e}-4$ & 3.00 & $0.26 \mathrm{e}-5$ & 4.15 \\
& $1 / 13$ & $0.71 \mathrm{e}-4$ & 2.04 & $0.21 \mathrm{e}-4$ & 3.00 & $0.12 \mathrm{e}-5$ & 4.11 \\
\hline
\end{tabular}

From (6.3), it is easy to see that $\boldsymbol{\beta}=-A^{-1}(B \boldsymbol{\alpha}+K \boldsymbol{\gamma})$. Substituting $\boldsymbol{\beta}=-A^{-1}(B \boldsymbol{\alpha}+$ $K \gamma)$ in (4.5b)-(4.5c), using (6.1)-(6.2) and the bases for $V_{h}$ and $\mathbf{W}_{h}$, (4.5b)-(4.5c) can be reformulated as: Find $[\boldsymbol{\gamma}, \boldsymbol{\alpha}]^{\prime}$ such that

$$
\begin{aligned}
& F_{i}^{1}(\boldsymbol{\gamma}, \boldsymbol{\alpha})=0 \text { for } 1 \leq i \leq N, \\
& F_{l}^{2}(\boldsymbol{\gamma}, \boldsymbol{\alpha})=0 \text { for } 1 \leq l \leq M,
\end{aligned}
$$

where

$$
F_{i}^{1}(\boldsymbol{\gamma}, \boldsymbol{\alpha})=\sum_{m=1}^{M} \gamma_{m}\left(-B_{m i}\right)+\sum_{j=1}^{N} \alpha_{j} J_{j i}-L_{i}
$$

$F_{l}^{2}(\boldsymbol{\gamma}, \boldsymbol{\alpha})=\int_{\Omega} a\left(\sum_{j=1}^{N} \alpha_{j} \phi_{j}\right)\left(\sum_{m=1}^{M}\left[-A^{-1}(B \boldsymbol{\alpha}+K \boldsymbol{\gamma})\right]_{m} \boldsymbol{\chi}_{m}\right) \cdot \chi_{l} d x-\sum_{m=1}^{M} \gamma_{m} A_{m l}$

where $\left[-A^{-1}(B \boldsymbol{\alpha}+K \boldsymbol{\gamma})\right]_{m}=-\sum_{j=1}^{N_{v}}\left(A^{-1} B\right)_{m, j} \alpha_{j}-\sum_{l=1}^{M}\left(A^{-1} K\right)_{m, l} \gamma_{l}$.

In order to solve the nonlinear algebraic system, we apply Newton's method. The Jacobian matrix $\mathcal{J}$ of the system takes the form

$$
\mathcal{J}=\left[\begin{array}{cc}
-B^{\prime} & J \\
-A & G
\end{array}\right]
$$

where $G=\left[g_{l i}\right]=\left[\partial F_{l}^{2} / \partial \alpha_{i}\right]$ and $B^{\prime}$ is the transpose of $B$.

Example. Set the nonlinear term $a(u)$ as $1+u^{2}$ and choose the load function $f$ so that the exact solution is $u=x(1-x) y(1-y)$. The initial guess for Newton's iteration is taken to be the zero solution. For this example, we consider the approximate solution obtained after 10 iterations. Note that, we have chosen the stabilization coefficient $C_{12}=0, C_{11}=O(\tau)$ and $C_{22}=O\left(\frac{1}{\tau}\right)$ for some $\tau \in \mathbb{R}$. The order of convergence for $e_{u}=u-u_{h}$ and $\mathbf{e}_{\boldsymbol{\sigma}}=\boldsymbol{\sigma}-\boldsymbol{\sigma}_{h}$ is computed for the cases $k=1$ and 2. We then compute the order of convergence for $e_{u}=u-u_{h}$ and $\mathbf{e}_{\boldsymbol{\sigma}}=\boldsymbol{\sigma}-\boldsymbol{\sigma}_{h}$ for the cases $k=1$ and 2 with different choices of $\tau$. In Table 1, we provide the history of convergence with the choices for $\tau=1$, that is, independent of mesh size. We 
provide history order of convergence in Table 2 choosing $\tau$ of order $h$. In Table 3 , we present history of convergence when $\tau$ is of order $\frac{1}{h}$. We expect to see the order of convergence predicted by Theorem 1.1 that is, $k+1$ for $\left\|\boldsymbol{\sigma}-\boldsymbol{\sigma}_{h}\right\|$ and the order of convergence predicted by Theorem 5.1. that is, $k+2$ for $\left\|u-u_{h}^{*}\right\|$, provided $k \geq 1$. We can see in Table 1, 2 and 3 that these orders of convergence are actually achieved in full agreement with the theory. We compare our results with Tables 2, 3 and 6 of [15] and observe that we obtain similar results as for linear selfadjoint elliptic case.

TABLE 3. History of Convergence for $\tau=O\left(\frac{1}{h}\right)$

\begin{tabular}{|c|c|cc|cc|cc|}
\hline & mesh & $\left\|u-u_{h}\right\|$ & & $\left\|\boldsymbol{\sigma}-\boldsymbol{\sigma}_{h}\right\|$ & & $\left\|u-u_{h}^{*}\right\|$ & \\
\hline $\mathrm{k}$ & $\mathrm{h}$ & error & order & error & order & error & order \\
\hline \multirow{4}{*}{1} & $1 / 3$ & $0.15 \mathrm{e}-1$ & - & $0.90 \mathrm{e}-1$ & - & $0.17 \mathrm{e}-1$ & - \\
& $1 / 5$ & $0.48 \mathrm{e}-2$ & 1.65 & $0.51 \mathrm{e}-1$ & 0.83 & $0.54 \mathrm{e}-2$ & 1.65 \\
& $1 / 7$ & $0.22 \mathrm{e}-2$ & 1.87 & $0.35 \mathrm{e}-1$ & 0.93 & $0.25 \mathrm{e}-2$ & 1.87 \\
& $1 / 9$ & $0.13 \mathrm{e}-2$ & 1.93 & $0.26 \mathrm{e}-1$ & 0.96 & $0.15 \mathrm{e}-2$ & 1.93 \\
& $1 / 11$ & $0.83 \mathrm{e}-3$ & 1.95 & $0.21 \mathrm{e}-1$ & 0.97 & $0.94 \mathrm{e}-3$ & 1.95 \\
& $1 / 13$ & $0.58 \mathrm{e}-3$ & 1.97 & $0.18 \mathrm{e}-1$ & 0.98 & $0.66 \mathrm{e}-3$ & 1.97 \\
\hline \multirow{4}{*}{2} & $1 / 3$ & $0.17 \mathrm{e}-2$ & - & $0.23 \mathrm{e}-1$ & - & $0.76-3$ & - \\
& $1 / 5$ & $0.22 \mathrm{e}-3$ & 3.01 & $0.64 \mathrm{e}-2$ & 1.85 & $0.10 \mathrm{e}-3$ & 2.88 \\
& $1 / 7$ & $0.64 \mathrm{e}-4$ & 3.01 & $0.29 \mathrm{e}-2$ & 1.93 & $0.30 \mathrm{e}-4$ & 3.00 \\
& $1 / 9$ & $0.27 \mathrm{e}-4$ & 3.00 & $0.16 \mathrm{e}-2$ & 1.96 & $0.13 \mathrm{e}-4$ & 3.03 \\
& $1 / 11$ & $0.14 \mathrm{e}-4$ & 2.99 & $0.11 \mathrm{e}-2$ & 1.97 & $0.64 \mathrm{e}-5$ & 3.04 \\
& $1 / 13$ & $0.80 \mathrm{e}-5$ & 2.99 & $0.74 \mathrm{e}-3$ & 1.97 & $0.37 \mathrm{e}-5$ & 3.04 \\
\hline
\end{tabular}

\section{Conclusion}

Based on the analysis in [15, we first obtain a superconvergence estimate for a nonselfadjoint linear elliptic problem. Then, we extend our analysis to a nonlinear elliptic problem. The results presented in this article improve the error estimates obtained in 19] for $\left\|\mathbf{q}-\mathbf{q}_{h}\right\|$ and $\left\|\boldsymbol{\sigma}-\boldsymbol{\sigma}_{h}\right\|$. Compared to [10] in which Chen has proposed a postprocessing scheme for a nonlinear elliptic problem using a nonlinear mixed method; see [10, (4.1)]. In this article, we have proposed a postprocessing which depends on linear equation (5.2) and we have shown that $u_{h}^{*}$ converges faster than $u_{h}$ with order of convergence $O\left(h^{k+2}\right)$ in $L^{2}$-norm.

\section{ACKNOWLEDGMENTS}

The second author acknowledges the financial support through Project No. 08DST012 funded by the Department of Science and Technology, Government of India. The third author (EJP) was partially supported by the WCU program through the Korea Science and Engineering Foundation funded by the Ministry of Education, Science, and Technology R31-2008-000-10049-0 and NRF-2010-0013374. The authors are thankful to both referees for their valuable suggestions and comments. 


\section{REFERENCES}

1. M. Ainsworth and D. Kay, The approximation theory for the p-version finite element method and application to the nonlinear elliptic PDEs, Numer. Math. 82 (1999), 351-388. MR.1692127 (2000i:65177)

2. M. Ainsworth and D. Kay, Approximation theory for the hp-version finite element method and application to the nonlinear Laplacian, Applied Numerical Mathematics, 34 (2000), 329-344. MR1782539 (2001e:65175)

3. D. N. Arnold, F. Brezzi, B. Cockburn and L. D. Marini, Unified analysis of discontinuous Galerkin methods for elliptic problems, SIAM J. Numer. Anal., 39 (2002), 1749-1779. MR.1885715 (2002k:65183)

4. I. Babuska and M. Suri, The h-p version of the finite element method with quasiuniform meshes, RIARO Model. Math. Anal. Numer., 21 (1987), 199-238. MR896241 (88d:65154)

5. S. C. Brenner, Poincaré-Friedrichs inequalities for piecewise $H^{1}$ functions, SIAM J. Numer. Anal., 41 (2003), 306-324. MR.1974504 (2004d:65140)

6. F. Brezzi and M. Fortin, Mixed and Hybrid Finite Element Methods, Springer-Verlag, (1991). MR.1115205 (92d:65187)

7. R. Bustinza and G. N. Gatica, A local discontinuous Galerkin method for nonlinear diffusion problems with mixed boundary conditions, SIAM J. Sci. Comput., 26 (2004), 152-177. MR2114338 (2005k:65201)

8. P. Castillo, Performance of discontinuous Galerkin methods for elliptic PDE's, SIAM J. Sci. Comput., 24(2002), 524-547. MR 1951054(2003m:65200)

9. P. Castillo, B. Cockburn, I. Perugia and D. Schötzau, An a priori error analysis of the local discontinuous Galerkin method for elliptic problems, SIAM J. Numer. Anal., 38(2000), 16761706. MR 1813251 (2002k:65175)

10. Z. Chen, Expanded mixed finite element methods for quasilinear second order elliptic problems. II, RAIRO Modal. Math. Anal. Numer., 32(1998), 501-520. MR1637069 (99j:65202)

11. P. G. Ciarlet, The Finite Element Method for Elliptic Problems, North-Holland Publishing Company, (1978). MR0520174 (58:25001)

12. B. Cockburn, B. Dong, J. Guzmán, A superconvergent LDG-hybridizable Galerkin method for second-order elliptic problems, Math. Comp., 77(2008), 1887-1916. MR2429868(2009d:65166)

13. B. Cockburn, J. Gopalkrishnan, H. Wang, Locally conservative fluxes for the continuous Galerkin method, SIAM J. Numer. Anal., 45(2007), 1742-1776. MR2338408 (2008g:65160)

14. B. Cockburn, J. Gopalkrishnan, R. Lazarov, Unified hybridization of discontinuous Galerkin, mixed, and continuous Galerkin methods for second order elliptic problems, SIAM J. Numer. Anal., 47(2009), 1319-1365. MR2485455(2010b:65251)

15. B. Cockburn, J. Guzmán, H. Wang, Superconvergent discontinuous Galerkin methods for second-order elliptic problems, Math. Comp. 78(2009), 1-24. MR2448694(2009i:65213)

16. B. Cockburn and C.-W. Shu, The local discontinuous Galerkin time-dependent method for convection-diffusion systems, SIAM J. Numer. Anal., 35 (1998), pp. 2440-2463. MR.1655854 (99j:65163)

17. J. Douglas and T. Dupont, A Galerkin method for a nonlinear Dirichlet problem, Math. Comp., 29(1975), 689-696. MR0431747 (55:4742)

18. T. Gudi, Amiya K. Pani, An hp-Discontinuous Galerkin method for quasilinear elliptic problems of nonmonotone type, SIAM J. Numer. Anal. 45(2007), 163-192. MR2285849 (2008c:65324)

19. T. Gudi, N. Nataraj, Amiya K. Pani, An hp-local discontinuous Galerkin method for some quasilinear elliptic boundary value problems of nonmonotone type, Math. Comp. 77(2008), 731-756. MR2373177 (2009d:65170)

20. F. A. Milner, Mixed finite element methods for quasilinear second-order elliptic problems, Math. Comp. 44 (1985), 303-320. MR777266 (86g:65215)

21. E.-J. Park, Mixed finite element methods for nonlinear second-order elliptic problems, SIAM J. Numer. Anal. 32 (1995), no. 3, 865-885. MR1335659 (96d:65187)

22. B. Riviere, M. F. Wheeler and V. Girault, A priori error estimates for finite element methods based on discontinuous approximation spaces for elliptic problems, SIAM J. Numer. Anal., 39(2001). 902-931. MR.1860450(2002g:65149)

23. M. F. Wheeler, A priori $L_{2}$ error estimates for Galerkin approximations to parabolic partial differential equations, SIAM J. Numer. Anal. 10 (1973), 723-759. MR0351124 (50:3613) 
Department of Mathematics, Industrial Mathematics Group, Indian Institute of Technology Bombay, Powai, Mumbai-400076

E-mail address: sangita@.iitk@gmail.com

Department of Mathematics, Industrial Mathematics Group, Indian Institute of Technology Bombay, Powai, Mumbai-400076

E-mail address: akp@math.iitb.ac.in

Department of Computational Science and Engineering-WCU, Yonsei University

E-mail address: ejpark@yonsei.ac.kr 